\title{
Dynamical correlations and collective excitations of Yukawa liquids
}

\author{
Z. Donkó ${ }^{1,2}+$, G. J. Kalman ${ }^{2}$, P. Hartmann ${ }^{1,2}$ \\ ${ }^{1}$ Research Institute for Solid State Physics and Optics, \\ Hungarian Academy of Sciences, POB 49, H-1525 Budapest, Hungary \\ ${ }^{2}$ Department of Physics, Boston College, Chestnut Hill, MA 02467, USA
}

\begin{abstract}
.
In dusty (complex) plasmas, containing mesoscopic charged grains, the grain-grain interaction in many cases can be well described through a Yukawa potential. In this Review we summarize the basics of the computational and theoretical approaches capable of describing many-particle Yukawa systems in the liquid and solid phases and discuss the properties of the dynamical density and current correlation spectra of three- and two-dimensional strongly coupled Yukawa systems, generated by molecular dynamics simulations. We show details of the $\omega(k)$ dispersion relations for the collective excitations in these systems, as obtained theoretically following the quasilocalized charge approximation, as well as from the fluctuation spectra created by simulations. The theoretical and simulation results are also compared with those obtained in complex plasma experiments.
\end{abstract}

\section{Introduction}

Strongly coupled plasmas - in which the average potential energy per particle dominates over the average kinetic energy - appear in a wide variety of physical systems: dusty plasmas, charged particles in cryogenic traps, condensed matter systems such as molten salts and liquid metals, electrons trapped on the free surface of liquid helium, astrophysical systems, such as the ion liquids in white dwarf interiors, neutron star crusts, supernova cores, and giant planetary interiors, as well as in degenerate electron or hole liquids in two-dimensional or layered semiconductor nanostructures [1. Many of these systems share some properties, which allows modeling them by considering explicitly only a single type of charged species and using a potential that accounts for the presence and effects of other types of species. This latter may be thought of as forming a charge-neutralizing background, which is either non-polarizable or polarizable. In the first case the interaction of the main plasma constituents can be expressed by the $\phi(r) \propto 1 / r$ Coulomb potential, while in the case of polarizable background the use of the $\phi(r) \propto \exp \left(-r / \lambda_{\mathrm{D}}\right) / r$ Yukawa potential is appropriate to account for screening effects ( $\lambda_{\mathrm{D}}$ is the Debye length). Perhaps the most important realizations of systems $\ddagger$ To whom correspondence should be addressed (donko@mail.kfki.hu) 
lending themselves to the approximation of the interaction by the Yukawa potential are charged colloids [2, 3, 4, 5] and dusty (complex) plasmas [6] (for comprehensive review on dusty plasmas see e.g. [7, 8]).

In the case of $2 \mathrm{D}$ colloidal systems the microscopic particles move in thin liquid films or between two closely separated glass plates.

In dusty plasmas both three-dimensional (3D) and two-dimensional (2D) settings appear in nature and in laboratory environments. In laboratory experiments $2 \mathrm{D}$ systems appear as a layer of dust particles levitated in gaseous discharges. While most of the studies on this latter system have been carried out in the crystalline state (for early references to "plasma crystals" see [9, 10, 11, 12]), the liquid state is receiving more current attention [13, 14, 15, 16, 17, 18]. The important difference between colloidal and dusty plasma systems is in the damping rate in particle dynamics and concomitantly in the wave dynamics. In colloidal suspensions the background liquid exerts a large friction on the moving charged particles, while in dusty plasmas the background is gaseous and therefore the friction is lower and the damping of the waves is weak. For this reason our focus in this Review will be directed at dusty plasmas. The Review will cover studies of strongly coupled plasmas mostly in the liquid state, where both free motion and localization intervene. The principal observation is that from the point of view of collective behavior it is the localization - even though imperfect localization, or quasilocalization - of particles that plays the principal role. In contrast to the Vlasov plasma where the collective modes arise from the fluid-like continuum behavior, in the strongly coupled liquid they are more related to the normal modes of the interacting quasilocalized particles. This, of course, suggests a link with the harmonic phonon theory of crystal lattices. At the same time, one has to allow for the randomness of the distribution of the particles and for the finite lifetime of the localization in the constantly changing potential landscape. This latter process is expected to be primarily responsible for the damping of the collective modes, in contrast both to Vlasov plasmas, where Landau damping dominates and to weakly correlated plasmas where collisional damping is the principal damping mechanism. This physical picture suggests a microscopic equation-of-motion model where the particles are trapped in local potential fluctuations. The particles occupy randomly located sites and undergo oscillations around them. At the same time, however, the site positions also change and a continuous rearrangement of the underlying quasi-equilibrium configuration takes place. Inherent in this description is the assumption that the two time scales are well separated and that for the description of the fast oscillating motion, the time average (converted into ensemble average) of the drifting quasi-equilibrium configuration is sufficient. Here the distinction between the "direct" and "indirect" thermal effects should be emphasized: the former are responsible for the actual motion and migration of the particles, the latter refer to the accessibility of the possible configurations of the random sites and to the temperature dependence of the probability of a particular configuration.

The interaction potential energy of particles in Yukawa liquids is given by (e.g. 
[19]):

$$
\phi(r)=\frac{Q^{2}}{4 \pi \varepsilon_{0}} \frac{\exp \left(-r / \lambda_{\mathrm{D}}\right)}{r},
$$

where $Q$ is the charge of the particles, $\varepsilon_{0}$ is the permittivity of free space, and the Debye length $\lambda_{\mathrm{D}}$ accounts for the screening of the interaction by other plasma species. The main (dimensionless) parameters, which fully characterize the systems are: (i) the coupling parameter (defined in the same way as for Coulomb systems):

$$
\Gamma=\frac{Q^{2}}{4 \pi \varepsilon_{0}} \frac{1}{a k_{\mathrm{B}} T},
$$

where $a$ is the Wigner-Seitz (WS) radius and $T$ is the temperature, and (ii) the screening parameter

$$
\kappa=\frac{a}{\lambda_{\mathrm{D}}} .
$$

$\Gamma$ is the customary measure of the ratio of the average potential energy to the average kinetic energy per particle; the strong coupling regime, relevant here, corresponds to $\Gamma \gg 1$. In the $\kappa \rightarrow 0$ limit the interaction reduces to the Coulomb type, while at $\kappa \rightarrow \infty$ it approximates the properties of a hard sphere interaction.

At $\kappa=0$, in 3D, the liquid domain is limited to coupling parameter values $\Gamma \lesssim 175$ [20, 21, 22], where the plasma is known to crystallize into a bcc lattice [23, 24]. In 2D, crystallization into hexagonal lattice occurs at a lower value of coupling, at $\Gamma \cong 137$, as found by computer simulations [25, 26] and proven by experiments [27] as well. At $\kappa>0,3 \mathrm{D}$ systems may crystallize either in a bcc or in a fcc lattice, depending on $\kappa$, as found by Hamaguchi et al. 28] in their calculation of the phase diagram of Yukawa systems. In 2D the crystallized form of the systems is always hexagonal.

The possibility of characterizing a Yukawa liquid with an effective coupling coefficient $\Gamma^{\star}$ instead of a $(\Gamma, \kappa)$ parameter pair has recently been addressed. In 3D $\Gamma^{\star}$ was derived by Vaulina, Fortov and co-workers [29, 30, 31] on the basis of the frequency of dust lattice waves. Subsequently, for 2D Yukawa liquids the $\Gamma^{\star}=f(\Gamma, \kappa)$ relationship was established by prescribing a constant amplitude for the first peak of the pair correlation function for fixed values of $\Gamma^{\star}[32]$. The solid-liquid melting line $\left(\Gamma_{\text {melting }}\right.$ vs. $\left.\kappa\right)$ in both $3 \mathrm{D}$ and $2 \mathrm{D}$ system was found in these studies to follow closely constant $\Gamma^{\star}$ values.

Additional characteristic parameters of the systems investigated here are the WS radius $a$ and the plasma frequency $\omega_{0}$, which are given for $3 \mathrm{D}$ and $2 \mathrm{D}$ systems by:

$$
\begin{aligned}
& a_{3 \mathrm{D}}=\left(4 n_{3 \mathrm{D}} \pi / 3\right)^{-1 / 3} \\
& a_{2 \mathrm{D}}=\left(n_{2 \mathrm{D}} \pi\right)^{-1 / 2},
\end{aligned}
$$

where $n_{3 \mathrm{D}}$ and $n_{2 \mathrm{D}}$ are the 3D (number) density and the 2D areal (number) density of particles, and

$$
\omega_{0,3 \mathrm{D}}=\sqrt{\frac{n_{3 \mathrm{D}} Q^{2}}{\varepsilon_{0} m}}
$$


and

$$
\omega_{0,2 \mathrm{D}}=\sqrt{\frac{n_{2 \mathrm{D}} Q^{2}}{2 \varepsilon_{0} m a_{2 \mathrm{D}}}} .
$$

Note that in 2D the nominal plasma frequency $\omega_{0}$ may also have different definitions, and some of the authors use the lattice constant instead of the WS radius as a length scale.

Other important frequencies characterizing strongly coupled plasmas are the Einstein frequencies, which are the normal modes of oscillation of a test charge in the presence of a given (static) distribution of charges. Einstein frequencies are well known for lattice structures, however, there has been relatively little work done on disordered and liquid phase systems [33]. Such systems are being studied through the combination of theoretical and simulation approaches [34, 35].

As to the possibilities of theoretical description, many-body systems can be treated theoretically in a straightforward way in the extreme limits of both weak interaction and very strong interaction. In the first case, one is faced with a gaseous system, or a Vlasov plasma, where correlation effects can be treated perturbatively $(\Gamma \ll 1)$. In the case of very strong interaction, the system crystallizes, the particles are completely localized and phonons are the principal excitations. In the intermediate regime - in the strongly coupled liquid phase - the localization of the particles in the local minima of the potential surface still prevails, however, due to the diffusion of the particles the time of localization is finite [34]. The localization of the particles (which may typically cover a period of several plasma oscillation cycles) serves as the basis of the Quasi-Localized Charge Approximation (QLCA) method [36, 37]. Besides the theoretical approaches computer simulations have proven to be invaluable tools for investigations of strongly coupled liquids of charged particles. Monte Carlo (MC) and molecular dynamics (MD) methods have widely been applied in studies of the equilibrium and transport properties, as well as of dynamical effects and collective excitations. The main difference between the two techniques is that in an MC simulation the particle configuration with the lowest energy is searched for, whereas MD simulations provide information about the time-dependent phase space coordinates of the particles, this way allowing studies of dynamical properties.

This paper intends to review the dynamical properties and collective behavior of strongly coupled Yukawa systems in the liquid and solid phases, in two and three dimensions. First we describe the numerical as well as theoretical methods used, in Sections 2 and 3, respectively. The analysis of the collective mode behavior of 3D liquids is presented in Section 4.1. In the $2 \mathrm{D}$ case we investigate both an ideally narrow particle layer, and a layer having a finite width, where particles are confined by an external parabolic potential. The analysis of these systems is described in Sections 4.2 and 4.3 , respectively. Section 5 gives a brief summary of the experimental studies relevant to the theoretical and simulation results reviewed. Section 6 gives a summary of the paper as well as a short outlook on the topics, which may have been additional 
subjects of this Review.

\section{Molecular dynamics simulations}

Molecular dynamics simulations follow the motion of particles by integrating their equations of motion while accounting for the pairwise interaction of the particles, as well as for the forces originating from any external field(s), see e.g. [38. In the plasma / gas background environment friction forces and randomly fluctuating forces also act on the particles in addition to the forces arising from the interaction of the electrically charged particles. The general form of the equation of motion (of a "test particle $i$ ) is (see e.g. [39]):

$$
m \ddot{\mathbf{r}}_{i}=\sum_{i \neq j} \mathbf{F}_{i, j}(t)+\mathbf{F}_{\text {ext }}(t)-m \eta \mathbf{v}_{i}(t)+\mathbf{R},
$$

where $\mathbf{F}_{i, j}$ is the force originating from the interaction with particle $j, \mathbf{F}_{\text {ext }}$ is the force originating from any external field, $\eta$ is the friction coefficient, and $\mathbf{R}$ represents a Brownian randomly fluctuating force (Langevin force). The results presented here correspond to "idealized" Yukawa liquids for which $\eta=0$ and $\mathbf{R}=0$ are assumed. Also, in most of our studies we investigate infinite (unconfined) systems, for which $\mathbf{F}_{\text {ext }}=0$, as well, although as an example a quasi-two-dimensional liquid - confined by an external parabolic field - is also studied. In the case of unconfined systems periodic boundary conditions (PBC) are imposed in the simulations. In the case of confinement along one of the coordinates, PBC-s are used in the unconfined directions. It is noted that for charged colloids Brownian molecular dynamics simulation [3, 40] is widely used, which represents the extreme limit of large friction and large $\mathbf{R}$. In this case the inertial term $\left(m \ddot{\mathbf{r}}_{i}\right)$ in eq. (8) is neglected.

The calculation of the force acting on a particle of the system, $\mathbf{F}_{i}$, is relatively simple in the case of short-range potentials (like the Lenard-Jones potential or the $1 / r^{12}$ potential). In this case MD methods make use of the truncation of the interaction potential thereby limiting the need for the summation of pairwise interactions around a test particle to a region of finite size. In the case of long-range interactions (e.g. Coulomb or low- $\kappa$ Yukawa potentials), which are also of interest here, however, such truncation of the potential is not allowed, and thus special techniques, like Ewald summation [41, have to be used in MD simulations. Besides the Ewald summation technique there exist few additional methods, like the fast multipole method and the particle-particle particlemesh method (PPPM, or P3M), which can be used to handle long-range interaction potentials, see e.g. [42]. The results presented here for Coulomb systems have been obtained from simulations using this latter method [43, 44, 45, 46, 47]. In the PPPM scheme the interparticle force is partitioned into (i) a force component $\mathbf{F}_{\mathrm{PM}}$ that can be calculated on a mesh (the "mesh force") and (ii) a short-range ("correction") force $\mathbf{F}_{\mathrm{PP}}$, which is to be applied to closely separated pairs of particles only. In the mesh part of the calculation charged clouds are used instead of point-like particles and their interaction is calculated on a computational mesh, taking also into account periodic images (for 
more details see [46, 47]). This way the PPPM method makes it possible to take into account periodic images of the system (in the PM part), without truncating the long range Coulomb or low- $\kappa$ Yukawa potentials. For screening values $\kappa \gtrsim 1$ the PP part alone provides sufficient accuracy. In these cases the mesh part of the calculation is not used, the interaction forces are summed for particles situated within a ( $\kappa$-dependent) cutoff radius around the test particle. Identification of these "neighboring" particles is aided by the "chaining mesh technique".

In the simulations presented here usually a spatially random particle configuration is set up at the initialization, with particle velocities sampled from a Maxwellian distribution of temperature $T_{0}$, which corresponds to the desired value of the coupling parameter $\Gamma$ [see eq. (2)]. The equations of motion of the particles are integrated using the leapfrog scheme or the velocity-Verlet scheme. The desired system temperature is reached by rescaling the particle momenta during an initialization phase of the simulation. In equilibrium MD simulations measurements on the system are taken following this phase, in the state of thermodynamic equilibrium. During this phase thermostation is usually no longer applied. If thermostation is necessary, rescaling of particle velocities is to be avoided, algorithms like the Nosé-Hoover thermostat can be applied (see e.g. [38, 48, 49]).

In our studies measurements on the system are taken at constant volume $(V)$, particle number $(N)$ and total energy $(E)$. The MD simulations directly provide the pair correlation function (PCF) of the system, which is the basis for the calculation of thermodynamic quantities (not detailed here, see e.g. [32]), and is also required as input to the QLCA equations for the calculation of the dispersion relations and other quantities (see later).

In the MD simulation information about the (thermally excited) collective modes and their dispersion is obtained from the Fourier analysis of the correlation spectra of the density fluctuations

$$
\rho(k, t)=k \sum_{j} \exp \left[i k x_{j}(t)\right]
$$

yielding the dynamical structure function as [50]:

$$
S(k, \omega)=\frac{1}{2 \pi N} \lim _{\Delta T \rightarrow \infty} \frac{1}{\Delta T}|\rho(k, \omega)|^{2},
$$

where $\Delta T$ is the length of data recording period and $\rho(k, \omega)=\mathcal{F}[\rho(k, t)]$ is the Fourier transform of (91).

Similarly, the spectra of the longitudinal and transverse current fluctuations, $L(k, \omega)$ and $T(k, \omega)$, respectively, can be obtained from Fourier analysis of the microscopic quantities

$$
\begin{aligned}
\lambda(k, t) & =k \sum_{j} v_{j x}(t) \exp \left[i k x_{j}(t)\right], \\
\tau(k, t) & =k \sum_{j} v_{j y}(t) \exp \left[i k x_{j}(t)\right],
\end{aligned}
$$


where $x_{j}$ and $v_{j}$ are the position and velocity of the $j$-th particle. Here we assume that $\mathrm{k}$ is directed along the $x$ axis (the system is isotropic) and accordingly omit the vector notation of the wave number. The way described above for the derivation of the spectra provides information for a series of wave numbers, which are multiples of $k_{\min }=2 \pi / H$, where $H$ is the edge length of the simulation box. The collective modes are identified as peaks in the fluctuation spectra. The widths of the peaks provide additional information about the lifetimes of the excitations: narrow peaks correspond to longer lifetimes, while broad features are signals for short lived excitations.

\section{Theoretical approaches}

The Molecular Dynamics calculations compute the dynamical density-density and current-current correlations (dynamical structure functions), from whose behavior the dispersion relations for the collective modes can be inferred. Following the same route in a theoretical analysis would be an extremely ambitious undertaking. Calculating the dynamical structure functions is not an easy task and not much progress has been achieved so far along this line. The single-particle and collective microscopic dynamics of a classical 3D Yukawa fluid was first analyzed by Barrat et al. [51], on the basis of memory function and mode-coupling theories. They have found that the longitudinal current fluctuations and the velocity autocorrelation function cross over continuously from the behavior characteristic of classical fluids with short-range interactions to the dynamics of a one-component plasma as the screening parameter $\kappa$ of the Yukawa potential is reduced.

2D Yukawa systems in the liquid phase were considered by Löwen [40] and Murillo and Gericke [52. In this latter work radial distribution functions have been computed with the hypernetted chain equations and were compared with those obtained from molecular dynamics simulations. The dynamical structure function obtained from the RPA approach extended by local field corrections was shown to be inadequate to reproduce the features of the structure function obtained from molecular dynamics. Ref. [40] focused mostly on the static properties and Brownian dynamics of the system, while also considering some features of the dynamical fluctuations. Applying the viscoelastic approximation Murillo [53] analyzed some aspects of the transverse current fluctuations.

Fortunately, for the determination of the collective mode spectrum a much more direct approach, via the analysis of the dielectric response (tensor) function, is available. Thus the primary goals of the analytical methods discussed below are the determination of the dielectric function and the derivation of the ensuing dispersion relation for the collective modes.

The dielectric tensor in the spatially homogeneous liquid phase is diagonal in the coordinate system, where $\mathbf{k}$ is along one of the coordinate axes. Accordingly, the collective modes can be classified by their polarization into longitudinal and transverse modes. In the crystalline solid phase the rotational symmetry is broken, the structure of the dielectric tensor is more intricate and the longitudinal and transverse polarizations 
do not, in general, represent eigenpolarizations anymore. In this Review we are concerned with the collective mode structure of the liquid phase, but the understanding of the behavior of the collective modes in the solid phase has a bearing, as we will discuss, on the formation of the collective modes in the strongly couple liquid phase as well.

\subsection{Fluctuation-Disspation Theorem}

The link between the $S(\mathbf{k}, \omega), L(\mathbf{k}, \omega), T(\mathbf{k}, \omega)$ spectra measured in the simulations and the dielectric function is provided by the Fluctuation-Dissipation Theorem

$$
\begin{aligned}
& S(\mathbf{k}, \omega)=\frac{k^{2}}{\omega^{2}} L(\mathbf{k}, \omega)=\frac{1}{\pi \beta n \omega} \operatorname{Im} \chi_{\mathrm{L}}(\mathbf{k}, \omega)=-\frac{1}{\pi \beta n \omega} \frac{\operatorname{Im} \bar{\chi}_{\mathrm{L}}(\mathbf{k}, \omega)}{\left|\varepsilon_{\mathrm{L}}(\mathbf{k}, \omega)\right|^{2}}, \\
& T(\mathbf{k}, \omega)=\frac{\omega^{2}}{k^{2}} \frac{1}{\pi \beta n \omega} \operatorname{Im} \chi_{\mathrm{T}}(\mathbf{k}, \omega),
\end{aligned}
$$

where $\beta=1 / k T, \chi_{\mu \nu}(\mathbf{k}, \omega)$ is the susceptibility tensor, and $\bar{\chi}_{\mu \nu}(\mathbf{k}, \omega)$ is the proper (or total) susceptibility tensor.

At the $\Omega$ value where the dispersion relation is satisfied, $\chi_{\mathrm{L}, \mathrm{T}}^{-1}(\mathbf{k}, \Omega)$ vanishes. This, in general, happens only at a complex frequency, the imaginary part of which being characteristic of the damping of the mode. Since the dynamical structure functions are plotted and analyzed for real frequencies only, $\chi_{\mathrm{L}, \mathrm{T}}^{-1}(\mathbf{k}, \Omega)$ reaches only a minimum at some value of the real $\omega$, which can be expected to be in the vicinity of the actual complex $\Omega$ : this is the frequency value that can be identified at which the peak of the fluctuation spectrum occurs.

\subsection{Dielectric Response Function}

The tensorial dielectric response function $\varepsilon_{\mu \nu}(\mathbf{k}, \omega)$ can be expressed either in terms of the susceptibility tensor $\chi_{\mu \nu}(\mathbf{k}, \omega)$ or the proper (or total) susceptibility tensor $\bar{\chi}_{\mu \nu}(\mathbf{k}, \omega)$ and the Fourier transform $\varphi(k)$ of the interaction potential (1). This latter depends on the dimensionality of the system. In 3D

$$
\varphi(k)=\frac{1}{\varepsilon_{0}} \frac{Q^{2}}{k^{2}+\kappa^{2}}
$$

and in $2 \mathrm{D}$

$$
\varphi(k)=\frac{1}{2 \varepsilon_{0}} \frac{Q^{2}}{\left(k^{2}+\kappa^{2}\right)^{1 / 2}} .
$$

Then

$$
\varepsilon_{\mu \nu}(\mathbf{k}, \omega)=\delta_{\mu \nu}-\varphi(k) \chi_{\mu \nu}(\mathbf{k}, \omega) .
$$

In the coordinate system where $\mathbf{k}$ is along the $z$ axis in 3D and along the $y$ axis in 2D, the isotropic liquid $\varepsilon_{\mu \nu}(\mathbf{k}, \omega)$ has the structure

$$
\varepsilon_{\mu \nu}(\mathbf{k}, \omega)=\left[\begin{array}{ccc}
\varepsilon_{\mathrm{T}}(\mathbf{k}, \omega) & 0 & 0 \\
0 & \varepsilon_{\mathrm{T}}(\mathbf{k}, \omega) & 0 \\
0 & 0 & \varepsilon_{\mathrm{L}}(\mathbf{k}, \omega)
\end{array}\right]
$$




$$
\varepsilon_{\mu \nu}(\mathbf{k}, \omega)=\left[\begin{array}{cc}
\varepsilon_{\mathrm{T}}(\mathbf{k}, \omega) & 0 \\
0 & \varepsilon_{\mathrm{L}}(\mathbf{k}, \omega)
\end{array}\right]
$$

and the dispersion relations for the collective modes are given by

$$
\begin{array}{ll}
\varepsilon_{\mathrm{L}}(\mathbf{k}, \omega)=0, & \text { (a) } \\
\varepsilon_{\mathrm{T}}^{-1}(\mathbf{k}, \omega)=0 . & \text { (b) }
\end{array}
$$

The longitudinal dielectric function has the immediate physical significance that it relates the externally imposed electric field to the total (external+polarization) field by $E_{\text {total }}(\mathbf{k}, \omega)=E_{\text {external }}(\mathbf{k}, \omega) / \varepsilon_{\mathrm{L}}(\mathbf{k}, \omega)$. In contrast, the transverse dielectric function has well-defined physical meaning only in terms of the full electrodynamics of the system [54]. Here, there is a certain degree of arbitrariness in the definition of $\varepsilon_{\mathrm{T}}(\mathbf{k}, \omega)$. A useful alternative formulation of the dispersion relations is in terms of the external susceptibility

$$
\begin{aligned}
& \chi_{\mathrm{L}}(\mathbf{k}, \omega)=\frac{\bar{\chi}_{\mathrm{L}}(\mathbf{k}, \omega)}{\varepsilon_{\mathrm{L}}(\mathbf{k}, \omega)}, \\
& \chi_{\mathrm{T}}(\mathbf{k}, \omega)=\bar{\chi}_{\mathrm{T}}(\mathbf{k}, \omega) .
\end{aligned}
$$

This allows expressing the condition for the collective excitation in the universal form

$$
\chi_{\mathrm{L}, \mathrm{T}}^{-1}(\mathbf{k}, \omega)=0 .
$$

$\bar{\chi}_{\mu \nu}(\mathbf{k}, \omega)$ embodies all the dynamical properties of the system, which stem partly from interparticle correlations, partly from the random motion of the particles. Over the past half century an immense effort has gone into the calculation of this quantity for Coulomb systems, both classical and quantum. Most of the work focused on weakly coupled $(\Gamma \ll 1)$ or moderately coupled $(1<\Gamma<10)$ systems. Interest in strongly coupled Coulomb and Yukawa systems is more recent [55, 56]. In the strongly coupled domain the dynamics is dominated by correlations. Here and in the sequel we will mostly ignore the effect of thermal motions on $\chi_{\mu \nu}(\mathbf{k}, \omega)$; some comments on how to abandon this simplification will be made later in this Section.

While our focus in this Review is on the strongly coupled liquid phase, it will be instructive and of interest to begin with an orientation based on the weakly coupled Random Phase Approximation (RPA) theory. The RPA or Vlasov description is based on the assumption that the mean field dominates the particle-particle interaction and correlations can be ignored. This is tantamount to taking $\bar{\chi}_{\mu \nu}(\mathbf{k}, \omega)$ as that of the noninteracting gas (although perhaps not quite obviously: for a discussion see e.g. [57]): $\bar{\chi}_{\mu \nu}(\mathbf{k}, \omega)=\chi_{0}(\mathbf{k}, \omega) \delta_{\mu \nu}$, which, with the neglect of thermal motion is

$$
\chi_{0}(\mathbf{k}, \omega)=\frac{n}{m} \frac{k^{2}}{\omega^{2}}
$$

This leads to the simple expressions for the elements of the dielectric tensor

$$
\begin{array}{ll}
\varepsilon_{\mathrm{L}}(\mathbf{k}, \omega)=\varepsilon_{\mathrm{T}}(\mathbf{k}, \omega)=1-\frac{\bar{k}^{2}}{\bar{k}^{2}+\kappa^{2}} \frac{\omega_{0,3 \mathrm{D}}^{2}}{\omega^{2}}, & 3 \mathrm{D} \\
\varepsilon_{\mathrm{L}}(\mathbf{k}, \omega)=\varepsilon_{\mathrm{T}}(\mathbf{k}, \omega)=1-\frac{\bar{k}^{2}}{\left(\bar{k}^{2}+\kappa^{2}\right)^{1 / 2}} \frac{\omega_{0,2 \mathrm{D}}^{2}}{\omega^{2}}, & 2 \mathrm{D}
\end{array}
$$


where $\omega_{0,3 \mathrm{D}}$ and $\omega_{0,2 \mathrm{D}}$ are the respective $3 \mathrm{D}$ plasma frequency and the $2 \mathrm{D}$ nominal plasma frequency defined in Eqs. (6) and (7); $\bar{k}=k a$.

The dispersion relations (and their small- $k$ approximations) for the 3D and 2D longitudinal modes follow immediately from (17):

$$
\begin{array}{ll}
\Omega_{0,3 \mathrm{D}}^{2}(\mathbf{k})=\omega_{0,3 \mathrm{D}}^{2} \frac{\bar{k}^{2}}{\bar{k}^{2}+\kappa^{2}} \approx \frac{\omega_{0,3 \mathrm{D}}^{2}}{\kappa^{2}} \bar{k}^{2}, & 3 \mathrm{D} \\
\Omega_{0,2 \mathrm{D}}^{2}(\mathbf{k})=\omega_{0,2 \mathrm{D}}^{2} \frac{\bar{k}^{2}}{\left(\bar{k}^{2}+\kappa^{2}\right)^{1 / 2}} \approx \frac{\omega_{0,2 \mathrm{D}}^{2}}{\kappa} \bar{k}^{2} . & 2 \mathrm{D}
\end{array}
$$

For $k \rightarrow 0$ the longitudinal mode is acoustic, i.e. $\omega_{\mathrm{L}}(k \rightarrow 0)=s k$, with the $3 \mathrm{D}$ and $2 \mathrm{D}$ acoustic velocities $s_{3 \mathrm{D}}$ and $s_{2 \mathrm{D}}$ :

$$
\begin{aligned}
& s_{3 \mathrm{D}}=\frac{\omega_{0,3 \mathrm{D}}}{\kappa}, \\
& s_{2 \mathrm{D}}=\frac{\omega_{0,2 \mathrm{D}}}{\sqrt{\kappa}} .
\end{aligned}
$$

Note that if we compare 3D and 2D systems with the same average interparticle distance,

the acoustic speed in $2 \mathrm{D}$ is different by a factor $\sqrt{\frac{2}{3}} \kappa$ than in $3 \mathrm{D}$. The acoustic behavior in $2 \mathrm{D}$ is of course at complete variance with the corresponding $k \rightarrow 0$ of an unscreened Coulomb plasma, i. e. the limit $\kappa=0$, where $\omega \propto \sqrt{k}$.

It is clear that there is no mode satisfying the transverse dispersion relation (17b): the mean field RPA model, which is devoid of correlations, cannot support a transverse shear wave, since shear is a fundamentally correlational phenomenon.

\subsection{Quasi Localized Charge Approximation}

While the RPA provides a description of the weakly coupled gas, the strongly coupled liquid state of a Coulombic or Yukawa system requires a different approach. There have been various attempts over the years to calculate dispersion relations and related quantities for such systems. Noteworthy approaches include the high frequency sum rule method [50], the application of the STLS (Singwi, Tosi, Land and Sjolander) technique originally developed for the electron gas in metals [58, 59], the memory function approach [60, 61] and the viscoelastic model [53, 62, 63, 64].

In the long run, from a practical perspective most of these methods have turned out to be problematic. The problems that occur vary: they range from weak theoretical foundation through being more appropriate for static than dynamical processes, to resulting in an unwieldy formalism. On the other hand, a method originally proposed by Kalman and Golden in [65] that has become known as the Quasilocalized Charge Approximation (QLCA) (for a review see [36, 37]) has led to quite a successful history of accomplishments. The measure of success in this context is (a) the ability to calculate from available static data dynamical quantities that lend themselves to comparison with numerical or laboratory experiments; (b) solid agreement with the outcomes of MD simulations; and (c) a good accord with the newly available laboratory experiments (still in a rather limited number) on complex plasma wave propagation. The following 
is a concise description of the QLCA method; for more details the reader is referred to [36].

The conceptual basis for the QLCA has been a model that implies the following assumptions about the behavior of a strongly coupled Coulomb or Yukawa liquid: (i) in the potential landscape within the many-body system deep potential minima form that are capable of trapping (caging) charged particles; (ii) a caged charge oscillates with a frequency that is determined both by the local potential and the interaction with the other (caged) particles in their instantaneously frozen positions; (iii) the potential landscape changes slowly to allow the charges to execute a fair number of oscillations; (iv) the escape from the cages of the particles is caused by the gradual disintegration of the caging environment; the time scale of this process is governed by the coupling strength $\Gamma$; $(\mathrm{v})$ the (time and velocity dependent) correlation between a selected pair of particles is well approximated by the (time and velocity independent) equilibrium pair correlation; (vi) the frequency spectrum calculated from the averaged (correlated) distribution of particles represents, in a good approximation, the average over the distribution of frequencies originating from the actual ensemble.

Hypotheses (i)-(iv) have undergone careful testing by a series of MD simulation experiments both for Coulomb and Yukawa systems, and both for 2D and 3D configurations [34, 35], which will be discussed in Section 4. The validity of hypothesis (v) has recently been called into question in relation to multicomponent systems. The short time evolution of the pair correlation function in the vicinity of a particle moving with respect to its environment can certainly be velocity dependent and anisotropic: it is now believed that it is this behavior that is responsible for some discrepancies between MD simulation results and QLCA predictions occurring in binary Coulomb and Yukawa systems. It is not believed, however, that this behavior would be problematic in a single component system. As to item (vi), the question of the dynamical frequency distribution in a liquid has received very little attention, either theoretically or experimentally (the record is better in relation to disordered crystals, where the problem has been posed and approximation schemes have been proposed, although in a language where the central role of the dispersion relation is obscured - see, e.g. [66]). The extension of the QLCA in this direction, while less than pressing, would be desirable.

The central quantity in the QLCA is the dynamical matrix, either in three dimensions $(\mathcal{D}=3)$ or in two dimensions $(\mathcal{D}=2)$ :

$$
D_{\mu \nu}(\mathbf{k})=-\frac{n}{m} \int \mathrm{d}^{\mathcal{D}} r M_{\mu \nu}(r)\left[\mathrm{e}^{i \mathbf{k} \cdot \mathbf{r}}-1\right] h(r),
$$

which is formally similar to the eponymous quantity in the harmonic theory of lattice phonons and is derived from the equation of motion of properly constructed collective coordinates. $M_{\mu \nu}(r)=\partial_{\mu} \partial_{\nu} \phi(r)$ is the dipole-dipole interaction potential associated with $\phi(r) . \quad D_{\mu \nu}(\mathbf{k})$ is a functional of the equilibrium pair correlation function (PCF) $h(r)$, or of its Fourier transform $h(\mathbf{k})$.

The longitudinal and a transverse elements of the dielectric tensor are now expressed 
in terms of corresponding elements of $D_{\mu \nu}(\mathbf{k})$ :

$$
\varepsilon_{\mathrm{L} / \mathrm{T}}(\mathbf{k}, \omega)=1-\frac{\Omega_{0}^{2}(\mathbf{k})}{\omega^{2}-D_{\mathrm{L} / \mathrm{T}}(\mathbf{k})} .
$$

Thus the $D_{\mathrm{L}}(\mathbf{k})$ and $D_{\mathrm{T}}(\mathbf{k})$ local field functions are the respective projections of $D_{\mu \nu}(\mathbf{k})$ [36]. $\Omega_{0}(\mathbf{k})$ is the $3 \mathrm{D}$ or $2 \mathrm{D}$ longitudinal mode frequency, found in (22). One should keep in mind that in spite of the universality of the expression (24) the explicit forms of the $3 \mathrm{D}$ and $2 \mathrm{D} \varepsilon_{\mathrm{L}}(\mathbf{k}, \omega)$-s are quite different.

We note that the input required in the calculations is the static pair correlation function (PCF). In earlier works PCF-s generated by the HNC (hypernetted chain [67]) technique have been used as input data of the QLCA formulae to calculate the dispersion relations. With the advent of computer simulation techniques it turned out to be both more expedient and more accurate to import simulation generated PCF-s in the theoretical calculations. The results of the theoretical calculations presented in this paper use PCF-s derived from molecular dynamics (MD) computations.

We now can examine the dispersion relations that emerge from (24) and (25) in conjunction with (17b) and (17b). We will consider both the 2D and 3D cases with the corresponding results for the dispersion relations displayed in figures 1 and 2 , respectively. Figure 3 will compare the sound velocities and Einstein frequencies for these two cases.

Turning first to the 2D case, the longitudinal dispersion relation becomes

$$
\begin{aligned}
\Omega_{\mathrm{L}}^{2}(\mathbf{k}) & =\Omega_{0}^{2}(\mathbf{k})+D_{\mathrm{L}}(\mathbf{k})=\Omega_{0}^{2}(\mathbf{k})-\frac{n}{m} \int \mathrm{d}^{2} r M_{\mu \nu}(r)\left[\mathrm{e}^{i \mathbf{k} \cdot \mathbf{r}}-1\right] h(r) \\
& =\omega_{0,2 \mathrm{D}}^{2}\left\{\frac{\bar{k}^{2}}{\left(\bar{k}^{2}+\kappa^{2}\right)^{1 / 2}}+\frac{\bar{k}^{2}}{2} \int_{0}^{\infty} \Lambda^{2 \mathrm{D}}(\bar{k} \bar{r}, \kappa \bar{r}) h(\bar{r}) \mathrm{d} \bar{r}\right\},
\end{aligned}
$$

where $\bar{r}=r / a$, and

$$
\Lambda^{2 \mathrm{D}}(x, y)=\frac{\mathrm{e}^{-y}}{x^{2}}\left\{\left(1+y+y^{2}\right)\left[1-J_{0}(x)\right]+3\left(1+y+y^{2} / 3\right) J_{2}(x)\right\} .
$$

$J_{0}$ and $J_{2}$ are Bessel functions of the first kind.

In the mode frequency in (26) the RPA solution and the additional correlational part expressed in terms of the pair correlation function $h(r)$ are clearly separated. The result can, however, be transformed into an alternate form, expressed entirely in terms of the pair distribution function $g(r)=1+h(r)$. By introducing the extended dynamical matrix $C_{\mu \nu}(\mathbf{k})$ :

$$
\begin{aligned}
\Omega_{\mathrm{L}}^{2}(\mathbf{k}) & =\frac{n}{m} \int \mathrm{d}^{2} r M_{\mathrm{L}}(r)\left[\mathrm{e}^{i \mathbf{k} \cdot \mathbf{r}}-1\right] g(r) \equiv C_{\mathrm{L}}(\mathbf{k}) \\
& =\omega_{0,2 \mathrm{D}}^{2} \frac{\bar{k}^{2}}{2} \int_{0}^{\infty} \Lambda^{2 \mathrm{D}}(\bar{k} \bar{r}, \kappa \bar{r}) g(\bar{r}) \mathrm{d} \bar{r} .
\end{aligned}
$$

This result shows that the RPA contribution can be interpreted in terms of the same physical model as the QLCA: in this unified formulation the RPA force experienced by the oscillating particle is due to the mean field $[h(r)=0]$ only. Moreover, a reflection 
on the origin of the "1" term in the integrand identifies it as the generator of the Einstein frequency, the frequency of oscillation of a single particle in the frozen immobile environment of the other particles (see more discussion below):

$$
\Omega_{\mathrm{E}}^{2}=\frac{n}{m} \int \mathrm{d}^{2} r M_{\mathrm{L}}(r) g(r)=\omega_{0,2 \mathrm{D}}^{2} \frac{1}{2} \int_{0}^{\infty} \frac{\mathrm{d} \bar{r}}{\bar{r}^{2}} \mathrm{e}^{-\kappa \bar{r}}\left[1+\kappa \bar{r}+(\kappa \bar{r})^{2}\right] g(\bar{r}) .
$$

The $k \rightarrow 0$ behavior of the longitudinal mode is still acoustic, but the correlations reduce the acoustic (sound) speed below its RPA value. For $k \rightarrow \infty$ the mode frequency approaches the Einstein frequency $\Omega_{\mathrm{E}}$. This limiting behavior is a remarkable feature of strongly coupled Coulomb and Yukawa liquids [68, 69].
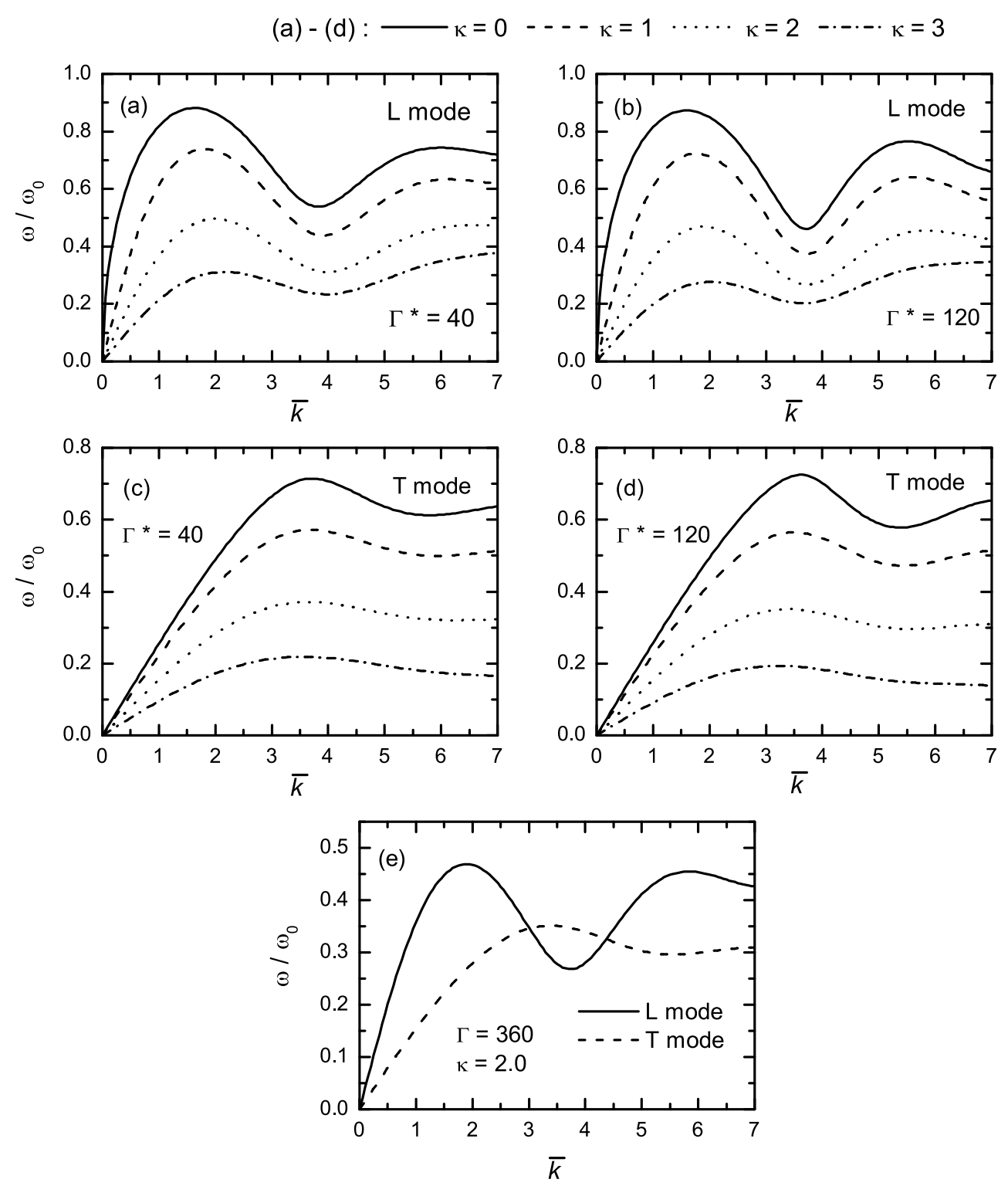

Figure 1. 2D Yukawa and Coulomb liquids: QLCA longitudinal and transverse dispersions for specified values of the effective coupling $\Gamma^{\star}$. 

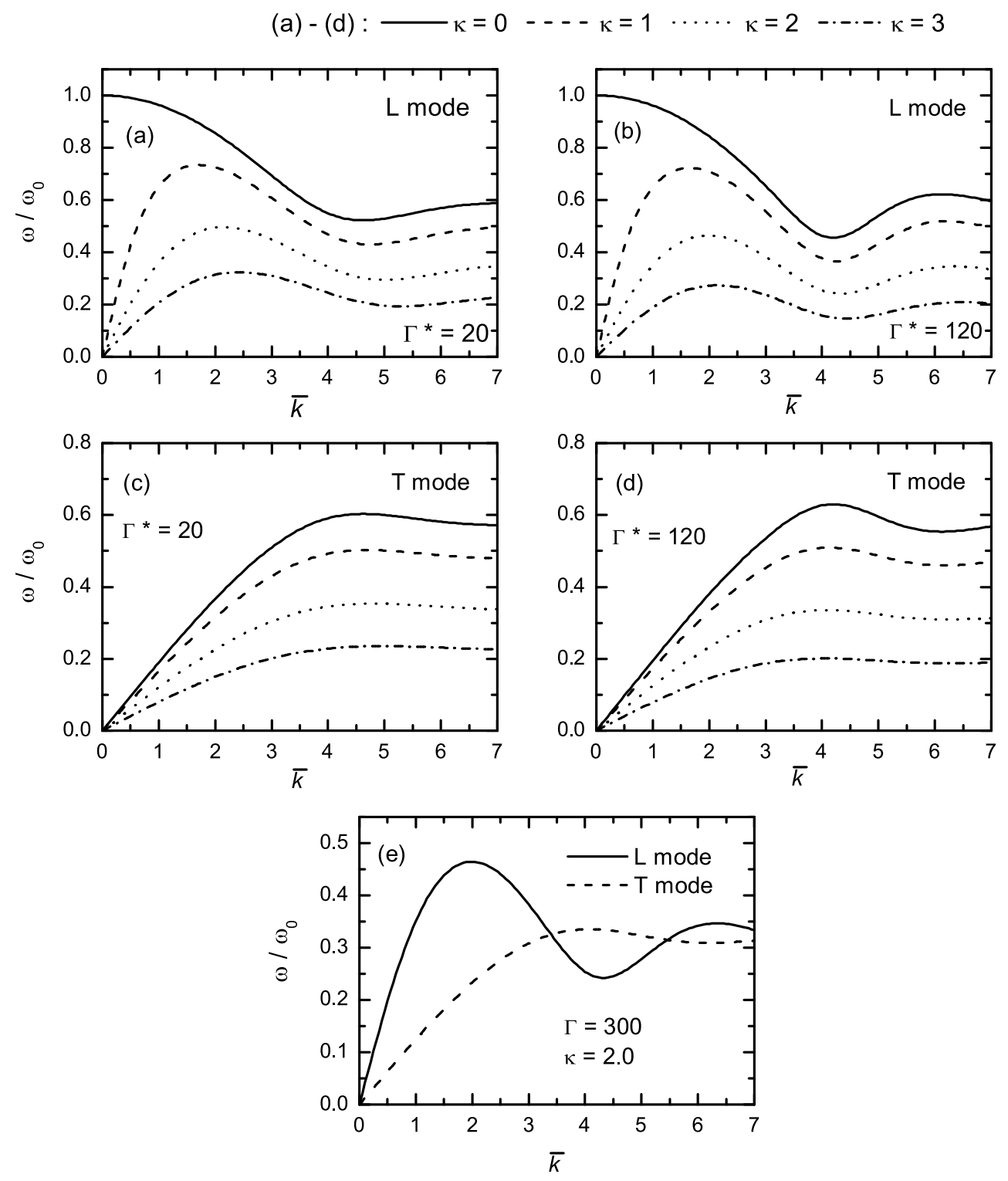

Figure 2. 3D Yukawa and Coulomb liquids: QLCA longitudinal and transverse dispersions for specified values of the effective coupling $\Gamma^{\star}$.

In contrast to the weakly coupled gas described through the RPA, the strongly coupled liquid supports a shear maintained transverse mode. This is reflected in the QLCA through the transverse dispersion relation

$$
\begin{aligned}
\Omega_{\mathrm{T}}^{2}(\mathbf{k}) & =D_{\mathrm{T}}(\mathbf{k}) \\
& =\omega_{0,2 \mathrm{D}}^{2} \frac{\bar{k}^{2}}{2} \int_{0}^{\infty} \Theta^{2 \mathrm{D}}(\bar{k} \bar{r}, \kappa \bar{r}) h(\bar{r}) \mathrm{d} \bar{r} \\
& =\omega_{0,2 \mathrm{D}}^{2} \frac{\bar{k}^{2}}{2} \int_{0}^{\infty} \Theta^{2 \mathrm{D}}(\bar{k} \bar{r}, \kappa \bar{r}) g(\bar{r}) \mathrm{d} \bar{r} \quad 2 \mathrm{D},
\end{aligned}
$$




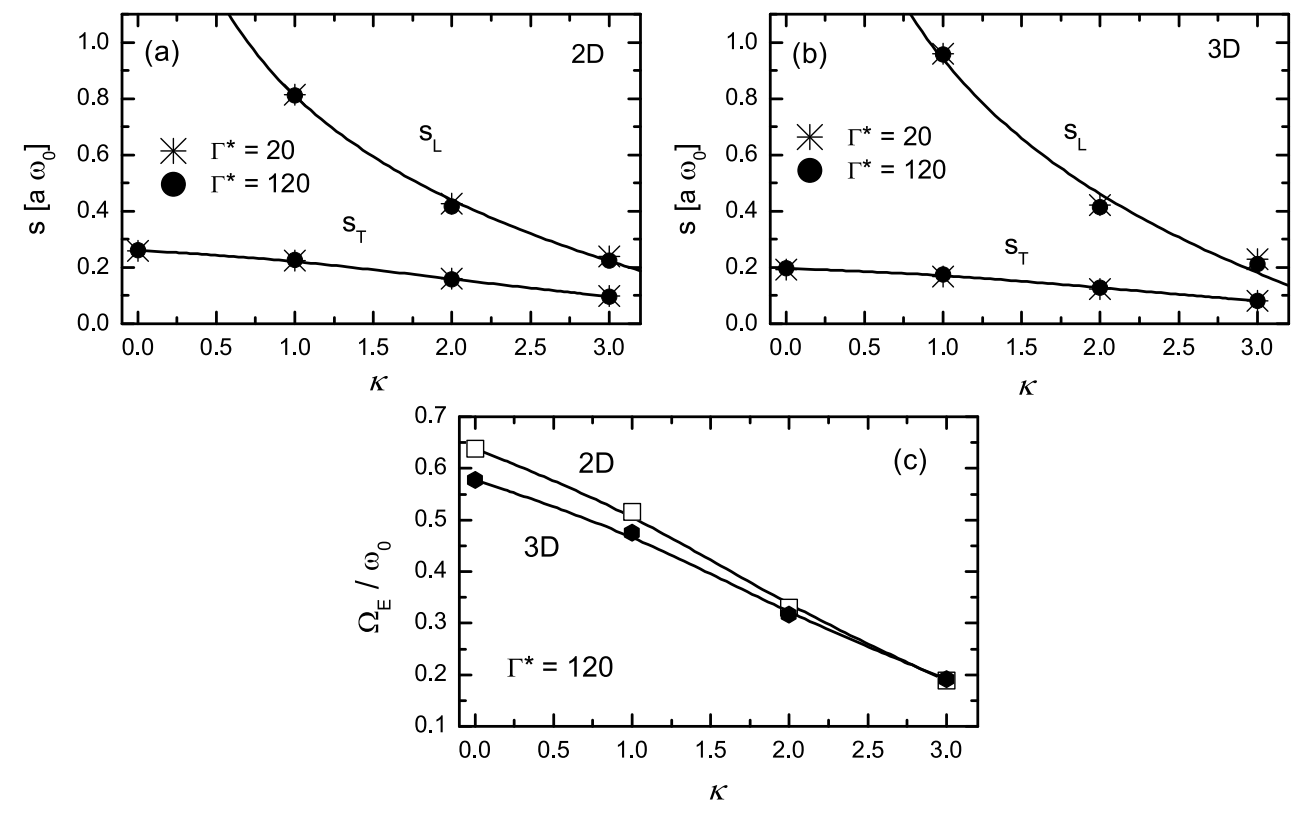

Figure 3. 2D and 3D Yukawa and Coulomb liquids: (a,b) QLCA sound velocities and (c) Einstein frequencies.

where

$$
\Theta^{2 \mathrm{D}}(x, y)=2 \frac{e^{-y}}{x^{2}}\left(1+y+y^{2}\right)\left[1-J_{0}(x)\right]-\Lambda^{2 \mathrm{D}}(x, y) .
$$

The last step in (30) follows from a simple algebraic identity and it reflects the absence of a mean transverse field in the medium.

The longitudinal and transverse dispersion curves for selected $\kappa$ and $\Gamma$ values are displayed in figure 1. The $k \rightarrow 0$ behavior of the transverse mode is also acoustic, but the correlation maintained acoustic speed is substantially below its longitudinal counterpart. However, the result that the transverse mode extends all the way to $k=0$ is spurious: the liquid is unable to support a shear wave in the uniform limit. The reason for this flaw is well understood: it has to be sought in the neglect of the migrationaldiffusional damping. The introduction of a phenomenological collision frequency [70] or of a semi-phenomenological extension of the QLCA (studied sofar only for the Coulomb case - see below) [68] provide an acceptable remedy.

For $k \rightarrow \infty$ the transverse mode also approaches the same Einstein frequency $\Omega_{\mathrm{E}}$ as the longitudinal mode, as dictated by the isotropy of the liquid.

Also shown are in figure 3 the $\kappa$ and $\Gamma$ dependences of the acoustic speeds and of the Einstein frequency. For moderate $\kappa$ values the sound velocities can also be obtained from the semi-analytic formulae [71]:

$$
s_{\mathrm{L}}^{2}=\frac{\omega_{0,2 \mathrm{D}}^{2} a^{2}}{\kappa}\left[1-\frac{\kappa}{2}\left(\frac{5}{8}-\frac{\kappa^{2}}{2} \frac{\partial}{\partial \kappa^{2}}+\frac{3 \kappa^{4}}{2} \frac{\partial^{2}}{\partial \kappa^{4}}\right) \frac{\beta\left|E_{\mathrm{c}}\right|}{\Gamma}\right]
$$




$$
s_{\mathrm{T}}^{2}=\frac{\omega_{0,2 \mathrm{D}}^{2} a^{2}}{2}\left(\frac{1}{8}-\frac{\kappa^{2}}{2} \frac{\partial}{\partial \kappa^{2}}-\frac{\kappa^{4}}{2} \frac{\partial^{2}}{\partial \kappa^{4}}\right) \frac{\beta\left|E_{\mathrm{c}}\right|}{\Gamma},
$$

where $E_{\mathrm{c}}=(n / 2) \int \phi(r)[g(r)-1] \mathrm{d} r^{2}$ is the correlation energy per particle.

Turning now to the 3D case, the formal results of the previous derivation can, mutatis mutandis, be taken over, with the understanding that the explicit forms of the RPA frequency $\Omega_{0}^{2}(\mathbf{k})$ and the kernels $\Lambda(\bar{k} \bar{r}, \kappa \bar{r})$ and $\Theta(\bar{k} \bar{r}, \kappa \bar{r})$ are different from their $2 \mathrm{D}$ counterparts.

$$
\begin{aligned}
\Omega_{0}^{2}(\mathbf{k})=\omega_{0,3 \mathrm{D}}^{2} & \frac{\bar{k}^{2}}{\bar{k}^{2}+\kappa^{2}} \quad 3 \mathrm{D} \\
\Lambda^{3 \mathrm{D}}(x, y)= & -2 \frac{\mathrm{e}^{-y}}{x}\left[\left(1+y+y^{2}\right)\left(\frac{\sin (x)}{x}+3 \frac{\cos (x)}{x^{2}}-3 \frac{\sin (x)}{x^{3}}\right)\right. \\
& \left.-\frac{y^{2}}{6}\left(1+3 \frac{\sin (x)}{x}+12 \frac{\cos (x)}{x^{2}}-12 \frac{\sin (x)}{x^{3}}\right)\right]
\end{aligned}
$$

and

$$
\Theta^{3 \mathrm{D}}(x, y)=\frac{1}{2}\left[\frac{\mathrm{e}^{-y}}{x} y^{2}\left(1-\frac{\sin (x)}{x}\right)-\Lambda^{3 \mathrm{D}}(x, y)\right] .
$$

There are now 2 degenerate transverse modes.

The expression for the Einstein frequency is also modified:

$$
\Omega_{\mathrm{E}}^{2}=\frac{n}{m} \int \mathrm{d}^{3} r M_{\mathrm{L}}(r) g(r)=\omega_{0,3 \mathrm{D}}^{2} \frac{\kappa^{2}}{3} \int_{0}^{\infty} \mathrm{d} \bar{r} \bar{r} \mathrm{e}^{-\kappa \bar{r}} g(\bar{r}) . \quad 3 \mathrm{D}
$$

The 3D longitudinal and transverse dispersion curves for selected $\kappa$ and $\Gamma$ values are displayed in figure 2. The $\kappa$ and $\Gamma$ dependences of the 3D acoustic speeds and of the Einstein frequency are shown together with their 2D counterparts in figure 3. Finally, for moderate $\kappa$ values the sound velocities can again be obtained from the semi-analytic formulae [70, 72 ]

$$
s_{\mathrm{L}}^{2}=\omega_{0,3 \mathrm{D}}^{2} a^{2}\left\{\frac{1}{\kappa^{2}}+\frac{2}{15} \int_{0}^{\infty} \bar{r} \mathrm{e}^{-\kappa \bar{r}}\left[1+\kappa \bar{r}+\frac{3}{4}(\kappa \bar{r})^{2}\right][g(\bar{r})-1] \mathrm{d} \bar{r}\right\}
$$

and

$$
s_{\mathrm{T}}^{2}=\omega_{0,3 \mathrm{D}}^{2} a^{2}\left\{-\frac{1}{15} \int_{0}^{\infty} \bar{r} \mathrm{e}^{-\kappa \bar{r}}\left[1+\kappa \bar{r}-\frac{1}{2}(\kappa \bar{r})^{2}\right][g(\bar{r})-1] \mathrm{d} \bar{r}\right\} .
$$

The results of a comparison between the dispersion properties of the collective modes in the $2 \mathrm{D}$ and $3 \mathrm{D}$ systems (assuming the same interparticle distance) may be summarized as follows.

(i) While for finite $\kappa$ values the qualitative behaviors of the two systems are very much the same, there is the well known fundamental difference in the $\kappa=0$ Coulomb limit between the 2D and 3D systems as to the small $k$ dispersion of the longitudinal mode: $\omega(k \rightarrow 0) \propto \sqrt{k}$ for $2 \mathrm{D}$, but in the $3 \mathrm{D}$ case $\omega(k=0)=\omega_{0,3 \mathrm{D}}$, the $3 \mathrm{D}$ plasma frequency. 
(ii) Not unrelated to this difference is the behavior of the longitudinal acoustic speeds at finite $\kappa$ values: since in $2 \mathrm{D} s \propto 1 / \sqrt{\kappa}$ and in $3 \mathrm{D} s \propto 1 / \kappa$, for small $\kappa$ the latter exceeds the former by the factor $\sqrt{3 / 2 \kappa}$.

(iii) In contrast, the transverse acoustic speeds exhibit only a mild $\kappa$ dependence and it is the $2 \mathrm{D}$ speed that is slightly higher than its $3 \mathrm{D}$ counterpart.

(iv) A similar 2D dominance prevails for the respective Einstein frequencies that govern the $k \rightarrow \infty$ behavior of the modes: in 2D the Einstein frequency assumes, for any $\kappa$, a somewhat higher value than in $3 \mathrm{D}$.

While (i) and (ii) are effects originating from the basic difference caused by the long range behavior of the Coulomb potential in a $2 \mathrm{D}$ vs. a $3 \mathrm{D}$ geometry and are already reflected in the RPA description, (iii) and (iv) are correlational phenomena and they point at the more important role the correlations play in $2 \mathrm{D}$ than in $3 \mathrm{D}$.

As a closing comment, it should be re-emphasized that the QLCA ignores possible damping mechanisms and Doppler shift, phase mixing, etc., due to the migrationaldiffusive motion of the particles and of velocity dispersion. A method has been recently proposed [73, 74, 75, 76] for the extension of the QLCA to take some of the neglected effects into account by combining the $D_{\mathrm{L}}(k), D_{\mathrm{T}}(k)$ as local field factors with the Vlasov density-density response. In this approximation

$$
\varepsilon_{\mathrm{L}, \mathrm{T}}=1-\frac{\varphi(k) \chi_{0, \mathrm{~L}, \mathrm{~T}}(\mathbf{k}, \omega)}{1-\varphi(k) \chi_{0, \mathrm{~L}, \mathrm{~T}}(\mathbf{k}, \omega)\left[D(\mathbf{k}) / \Omega_{0}^{2}(\mathbf{k})\right]},
$$

where $\chi_{0, \mathrm{~L}, \mathrm{~T}}(\mathbf{k}, \omega)$ is the longitudinal (transverse) Vlasov density response function of non-interacting particles. The application of this formalism to Yukawa systems has not been done yet, but in an early work [68] it was shown that in a 2D Coulomb system the combined effect of phase mixing and Landau-damping leads to the elimination of oscillations in the dispersion curve. The effect of Landau damping, which is not expected to play a major role at high $\Gamma$ values, is probably overestimated in this work.

\subsection{Lattice phonons}

With the caveat that the present Review addresses primarily the strongly coupled liquid state, it will be still useful to provide an overview of the phonon dispersion in a $2 \mathrm{D}$ or 3D Yukawa crystal. Such an overview will help to understand the structure of the liquid state in terms of a model resembling a disordered lattice and to view the collective modes in the liquid as being akin to the phonon excitations in the lattice.

The phonon dispersion is traditionally calculated in terms of the lattice dynamical matrix defined as

$$
C_{\mu \nu}(\mathbf{k})=-\frac{1}{m} \sum_{i, j} M_{\mu \nu}\left(\mathbf{r}_{i}-\mathbf{r}_{j}\right)\left[\mathrm{e}^{-i \mathbf{k} \cdot\left(\mathbf{r}_{i}-\mathbf{r}_{j}\right)}-1\right],
$$

with a summation over all the lattice sites $j$, keeping $i$ fixed $\left(\mathbf{r}_{i}=0\right)$. The resemblance to the extended QLCA dynamical matrix is not accidental. In contrast to the QLCA equivalent, however, the lattice dynamical matrix reflects the symmetry of the 
underlying lattice and not the rotational invariance of the isotropic liquid. Nevertheless, a dielectric tensor can be constructed along the same line in terms of the matrix $D_{\mu, \nu}(\mathbf{k})$, which is defined now as $C_{\mu, \nu}(\mathbf{k})$ with its mean field contribution removed

$$
D_{\mu \nu}(\mathbf{k})=C_{\mu \nu}(\mathbf{k})+\frac{1}{m} \int \mathrm{d}^{D} r M_{\mu \nu}(\mathbf{r})\left[\mathrm{e}^{-i \mathbf{k} \cdot \mathbf{r}}-1\right] .
$$

This leads to a structure analogous to (25):

$$
\varepsilon_{\mu \nu}(\mathbf{k}, \omega)=\delta_{\mu \nu}-\left[\frac{\Omega_{0}^{2}(\mathbf{k})}{\mathbf{1}-\mathbf{D}(\mathbf{k})}\right]_{\mu \nu} .
$$

The diagonalization of $\varepsilon_{\mu \nu}$ (or of $C_{\mu \nu}$ ) is now possible in the coordinate system of the eigenvectors, whose orientations, in general, do not coincide either with the direction of $\mathbf{k}$ or with the crystallographic axes.

To find the eigenmodes one can follow the traditional method (see e. g. [77, 78]) of solving the secular equation

$$
\left\|\omega^{2}-C_{\mu \nu}(\mathbf{k})\right\|=0
$$

or continue to follow the path of working with the dielectric tensor. This latter approach ensures that continuity with the liquid and RPA formalism is maintained. The dispersion relation in terms of $\varepsilon_{\mu, \nu}$ becomes

$$
k_{\mu} \varepsilon_{\mu \nu} k_{\nu}=0
$$

an obvious generalization of $(17 \mathrm{a})$. In fact, except in the degenerate isotropic case, it also includes the transverse relation (17b).

The 2D Yukawa system crystallizes in a triangular (hexagonal) lattice. The phonon spectrum was first calculated by Peeters and $\mathrm{Wu}$ [80], followed by Wang et al. [81]; a definitive calculations of the dispersion and the polarization for all propagation angles are given in [79, 82]. These results are shown in figure 4. The mode polarizations are purely longitudinal or transverse for propagation along the crystallographic axes $\left(\varphi=0^{\circ}\right.$ and $30^{\circ}$ ) only, otherwise they are mixed as shown in the figure. $\varphi$ is the propagation angle measured from the axis pointing towards the nearest neighbor. The angle $\Theta$ indicated in figure 4 is the polarization angle measured with respect to the propagation vector $\mathbf{k}$. The dispersion curves are periodic in $k$, but the period is simply the reciprocal lattice constant only along $0^{\circ}$ and $30^{\circ}$; for intermediate angles it is much longer, given by the formula

$$
\bar{k}_{0}=\frac{4 \pi}{\sqrt{3}} \sqrt{p^{2}+p q+q^{2}}
$$

where $p$ and $q$ are minimal integers satisfying

$$
\tan \left(\frac{\pi}{6}-\varphi\right)=\sqrt{\pi} \frac{p}{p+2 q}
$$

The dispersion curves of the lattice and those of the strongly coupled liquid do not show much resemblance. Yet, if one views the liquid as an aggregate of locally ordered domains whose symmetry axes are randomly distributed, then the similarity to 

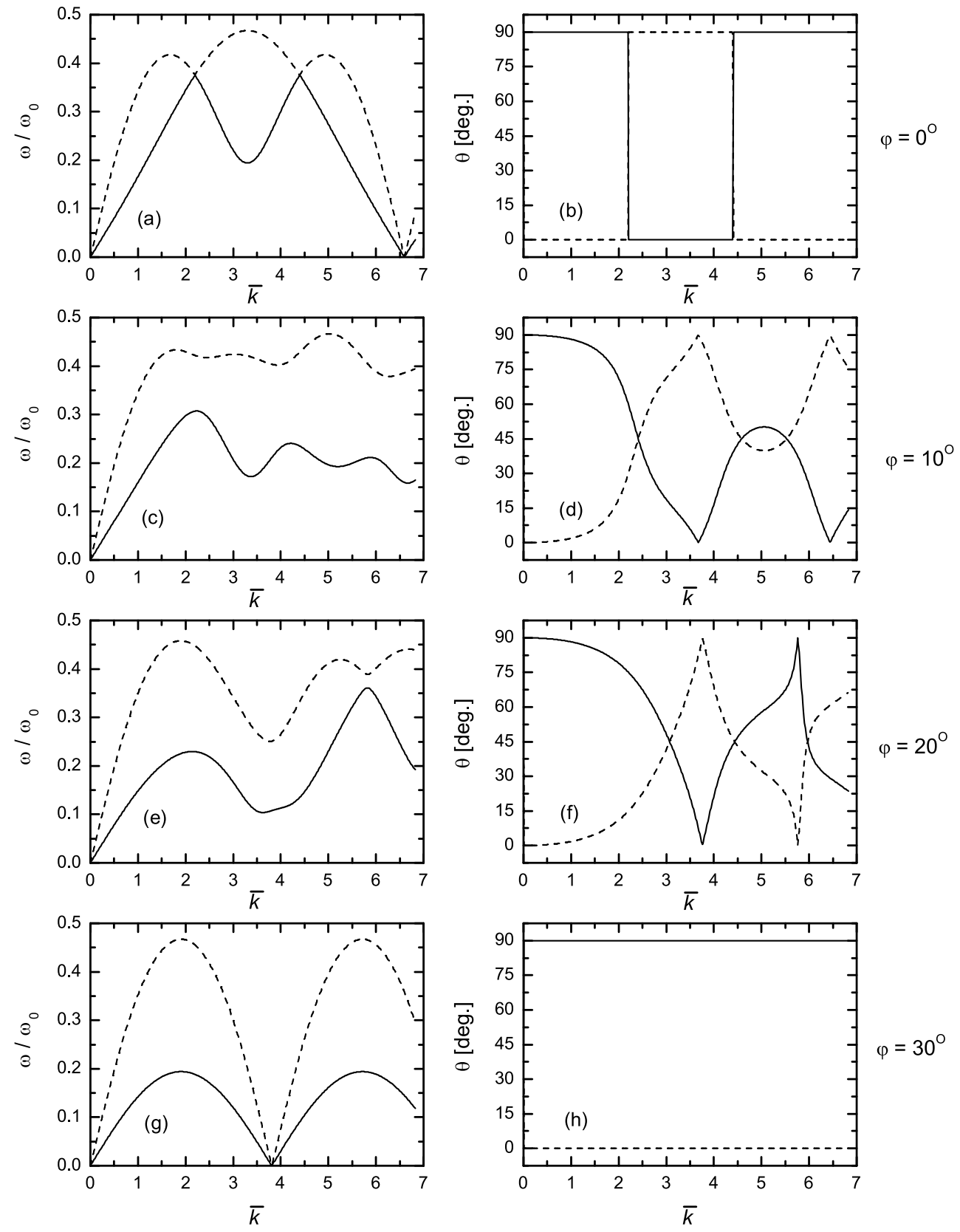

Figure 4. 2D Yukawa system: lattice dispersion curves and polarizations $(\kappa=2)$ for different angles of propagation. $\varphi$ is measured from the axis pointing towards the nearest neighbor, $\Theta$ is the polarization angle measured with respect to the propagation vector k. Partly reproduced from Ref. [79], copyright (2006) by Institute of Physics Publishing.

the liquid dispersion should be sought in a suitably averaged dispersion of the lattice. The strong angular dependence of the period $k_{0}$ suggests that an angular average should generate through phase mixing a smooth dispersion. This was carried out by projecting out the longitudinal and transverse components of the eigenmodes and comparing their respective angular averages with the longitudinal and transverse liquid modes [82]. 


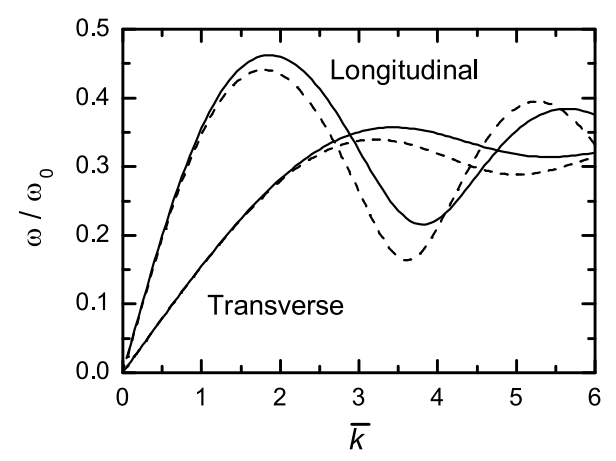

Figure 5. 2D Yukawa system: angularly averaged lattice (dashed lines) and QLCA (solid lines) dispersions of longitudinal and transverse modes using pair-correlation $[g(r)]$ data from MD simulation at $\Gamma=360$ and $\kappa=2$. Reproduced from Ref. [79, copyright (2006) by Institute of Physics Publishing.

Figure 5 shows that the agreement is quite reassuring. In principle, of course, one has to distinguish between the spectrum of an average of configurations and the average of the spectra of each of the configurations: that this observation notwithstanding the similarity persists can be taken as an indication that the sizes of the ordered domains in the liquid state are sufficiently large to diminish the effect of interaction between the domains.

The 3D Yukawa system crystallizes in a bcc or a fcc lattice (depending on the value of $\kappa$ ). A phase diagram has been given by Hamaguchi et al. [28]. Due to the existence of 3 rather than 2 eigenmodes and to their dependence both on the azimuthal and the polar angles of propagation a much more complex phonon spectrum is expected than in 2D. So far no systematic published study of this spectrum seems to exist; in an unpublished work, however, Sullivan, Kalman and Kyrkos [83] have generated a series of dispersion and polarization diagrams. A sample of these, for a number of $\varphi$ and $\psi$ angles, including propagations along the principal crystallographic axes is given in figure 6. ( $\Theta$ is the polarization angle measured with respect to the propagation vector $\mathbf{k}, \varphi$ is the polar angle in the $(x, y)$ plane and $\psi$ is the azimuthal angle measured from the $z$ axis.) Since no averaging has been performed, their comparison with the liquid spectrum at the present time is difficult. 

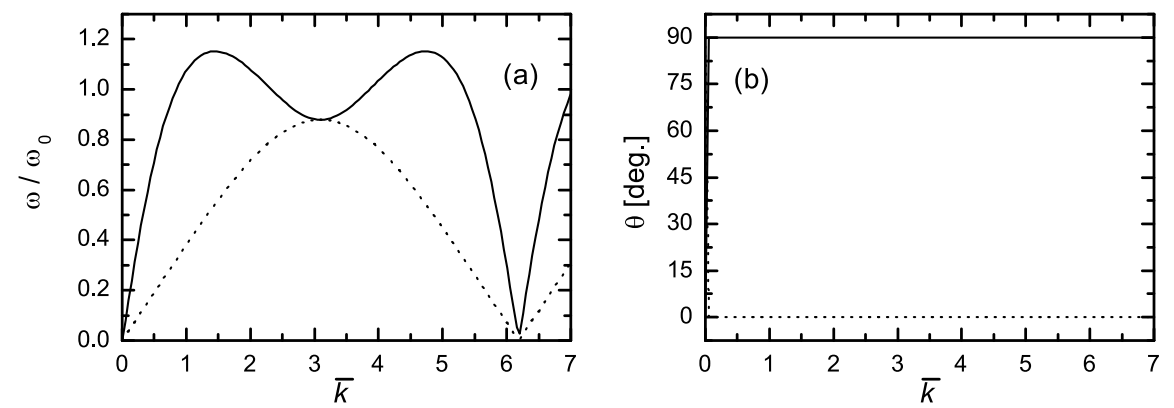

$x$ axis
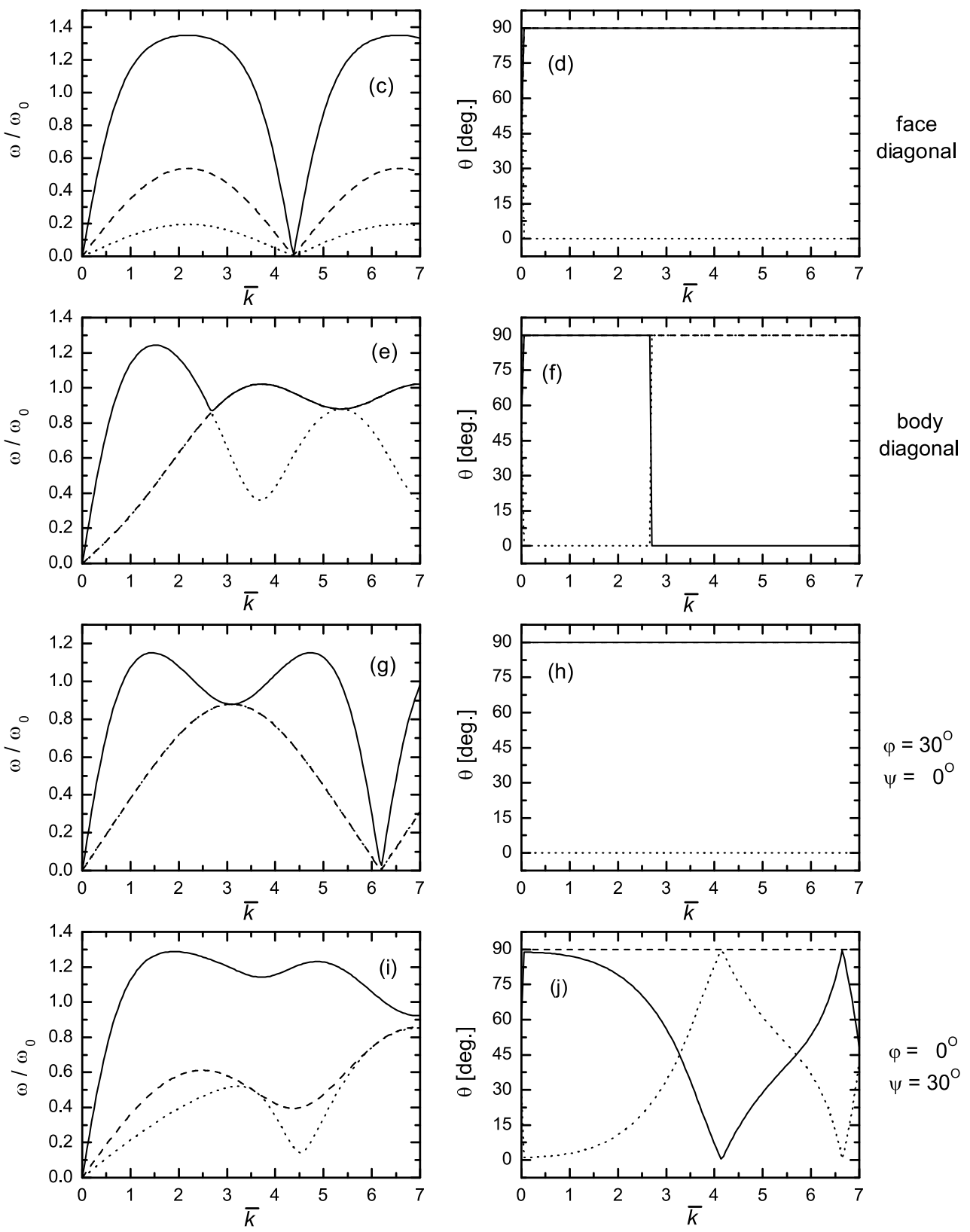

Figure 6. 3D Yukawa system at $\kappa=1$ : bcc lattice dispersion curves (left column) and polarizations (right column). $\Theta$ is the polarization angle measured with respect to the propagation vector $\mathbf{k}$. Continuous line: longitudinal; dashed line: first transverse; dotted line: second transverse polarizations. Note that the two transverse polarizations may be, but in general are not degenerate. $\varphi$ is the polar angle in the $(x, y)$ plane and $\psi$ is the azimuthal angle measured from the $z$ axis. 


\subsection{Einstein frequencies}

In addition to the collective excitations, Einstein frequencies represent a dynamical manifestation of the strong interaction in Yukawa systems. Einstein frequencies, as noted above, are the frequencies of oscillation of a single particle of the system (the "test particle") around its equilibrium position in the immobilized frozen environment of the other particles of the system. For obvious reasons, from the experimental point of view the "freezing" of the system but one particle is not a realistic proposition.

Thus until the advent of dusty plasma experiments Einstein frequencies were considered more of a theoretical construct than an observable quantity. The realization, however, that in the strongly coupled liquid state (but not in the crystalline solid) they represent the asymptotic $k \rightarrow \infty$ limit of the mode dispersion has promoted the Einstein frequency to the rank of observable quantities [84, 85].

In the crystalline solid state, where the test particle occupies a lattice site, the assumption that the potential experienced by the test particle is a quadratic function of the coordinates with a positive definite second derivative is in accord with the basic model of the harmonic theory of phonons. The maximum number of eigenfrequencies of oscillation is equal to the dimensionality of the system $(\mathcal{D}=\in$ or $\mathcal{D}=\ni)$; because of the lattice symmetry induced degeneracy the actual number may be less than $\mathcal{D}$. In a disordered lattice the degeneracy is removed and the frequencies depend on the actual realization of the disorder. In this case one has to distinguish between the "microscopic" Einstein frequencies $\left(\omega_{\mathrm{E}}\right)$ each of which is generated by a particular realization of the disorder and characterized by a distribution over the ensemble, and their ensemble average $\Omega_{\mathrm{E}}=\sqrt{\left\langle\omega_{\mathrm{E}}^{2}\right\rangle}$. It is this latter that will be continued to be referred to as "Einstein frequency" in the rest of this paper.

In addition, it is useful to consider the quantity

$$
\bar{\omega}_{\mathrm{E}}^{2}=\sum_{i=1}^{\mathcal{D}} \omega_{\mathrm{E}, i}^{2},
$$

i.e. the sum of the squared eigenfrequencies in a particular realization. Obviously, $\left\langle\omega_{\mathrm{E}}^{2}\right\rangle=\left\langle\bar{\omega}_{\mathrm{E}}^{2}\right\rangle$ but the distributions of $\omega_{\mathrm{E}}^{2}$ and $\bar{\omega}_{\mathrm{E}}^{2}$ can be quite different.

In a strongly coupled liquid the very notion of "equilibrium position" is questionable. Nevertheless, the "quasilocalization" condition, the basic tenet of the QLCA, is well satisfied for high $\Gamma$ values, as demonstrated by MD experiments [34] the details of which

will be discussed below. It is in this sense that the notion of the Einstein frequency and its distribution can be extended to the case of the strongly coupled liquid.

In a 3D Coulomb crystal the Einstein frequency is determined solely by the background, unaffected by the distribution of the (frozen) particles. This is the consequence of Gauss Theorem which, in turn, follows from the Poisson Equation that the $3 \mathrm{D}$ Coulomb potential satisfies. In this case

$$
\omega_{\mathrm{E}}^{2}=\frac{1}{3 \varepsilon_{0}} \frac{Q^{2} n}{m}=\frac{1}{3} \omega_{0,3 \mathrm{D}}^{2}
$$


In a disordered lattice or in a liquid (48) is not valid anymore; it is replaced by the weaker statement

$$
\bar{\omega}_{\mathrm{E}}^{2}=\frac{1}{3} \omega_{0,3 \mathrm{D}}^{2},
$$

the so-called Kohn Sum Rule [86], which also follows from the Poisson Equation.

Thus, in a disordered system while $\omega_{E}^{2}$ has a spread, $\bar{\omega}_{E}^{2}$ does not. As to the average, (49) is of course also tantamount to

$$
\Omega_{\mathrm{E}}^{2}=\frac{1}{3} \omega_{0,3 \mathrm{D}}^{2}
$$

For a genuine Yukawa potential the situation is quite different. The Yukawa potential satisfies the screened Poisson Equation rather than the Poisson Equation. A useful statement can be made now only for $\Omega_{\mathrm{E}}$, which now can be expressed in terms the average of the Yukawa potential $\langle\phi\rangle$ as experienced by the test particle at $\mathbf{r}=0$ [33]:

$$
\begin{aligned}
\Omega_{\mathrm{E}}^{2} & =\frac{\kappa^{2}}{3 m}\langle\phi\rangle \\
& =\omega_{0,3 \mathrm{D}}^{2} \frac{\kappa^{2}}{3} \int_{0}^{\infty} \mathrm{d} \bar{r} \bar{r} \mathrm{e}^{-\kappa r} g(\bar{r}) \\
& =\omega_{0,3 \mathrm{D}}^{2} \frac{1}{3}\left[1+\kappa^{2} \int_{0}^{\infty} \mathrm{d} \bar{r} \bar{r} \mathrm{e}^{-\kappa r} h(\bar{r})\right] .
\end{aligned}
$$

(51) is in agreement with (36), the result obtained from the QLCA. The third line clearly shows that, remarkably, in the $\kappa \rightarrow 0$ Coulomb limit the Yukawa Einstein frequency reduces to the background induced (50), even though the Yukawa system exists without any background. It can also be noted that $\frac{1}{2}\langle\phi\rangle=E_{\text {int }}$ is the interaction energy density of the system (with [positive] Hartree plus [negative] correlation contributions). Since the energy is the lowest in the ordered state, the Einstein frequency must increase with increasing disorder. According to the known phase diagram of the 3D Yukawa system [28] - as already mentioned - the system crystallizes in a bcc or a fcc lattice. The corresponding Einstein frequencies [87]

$$
\begin{aligned}
& \Omega_{\mathrm{E}}^{2}(\kappa=0)=0.33333 \omega_{0,3 \mathrm{D}}^{2} \\
& \Omega_{\mathrm{E}}^{2}(\kappa=1)=0.22293 \omega_{0,3 \mathrm{D}}^{2} \\
& \Omega_{\mathrm{E}}^{2}(\kappa=2)=0.09416 \omega_{0,3 \mathrm{D}}^{2}
\end{aligned}
$$

constitute an absolute lower bound.

In the 2D Coulomb system Gauss Theorem does not apply, the background plays no role and neither the Poisson Equation nor its screened variant is satisfied. Consequently, the Einstein frequency is determined by the distribution of the surrounding particles, both for Yukawa and Coulomb systems. In general

$$
\Omega_{\mathrm{E}}^{2}=\frac{1}{m}\left\langle M_{\mu, \nu}(r=0)\right\rangle=\omega_{0,2 \mathrm{D}}^{2} \int_{0}^{\infty} \frac{\mathrm{d} \bar{r}}{\bar{r}^{2}} \mathrm{e}^{-\kappa \bar{r}}\left[\frac{1}{2}(1+\kappa \bar{r})+(\kappa \bar{r})^{2}\right] g(\bar{r})
$$

in agreement with the QLCA result (29). 
An argument similar to the one discussed in relation to the 3D case leads to the conclusion that here also the ordered state exhibits the lowest Einstein frequency. The lattice structure is now hexagonal, for which

$$
\begin{aligned}
& \Omega_{\mathrm{E}}^{2}(\kappa=0)=0.39925 \omega_{0,2 \mathrm{D}}^{2} \\
& \Omega_{\mathrm{E}}^{2}(\kappa=1)=0.34433 \omega_{0,2 \mathrm{D}}^{2} \\
& \Omega_{\mathrm{E}}^{2}(\kappa=2)=0.24347 \omega_{0,2 \mathrm{D}}^{2}
\end{aligned}
$$

These values constitute then the lowest bound for the 2D Einstein frequencies.

In addition to the frequencies, the Einstein oscillations are also characterized by their eigenpolarizations. It is the distribution of the polarization angles which is of interest; this question has been investigated, however, only for the 2D case [82. In the perfect hexagonal lattice the degeneracy of the eigenmodes renders this distribution isotropic. It is also isotropic in the liquid phase. However, in the intermediate range where the lattice disorder develops the degeneracy for the microscopic eigenmodes is removed and the rotational invariance of the distribution is reduced to the sixfold symmetry of the underlying lattice. More will be shown about this remarkable effect in Section 4.2 .

\section{Simulation results}

In this Section we review the results of the extensive MD simulation work carried out since the beginning of this decade on the dynamical properties of Yukawa liquids. Most of the work was motivated by the QLCA theory and accordingly a great portion of the results pertaining to the areas where QLCA predictions are available are accompanied by comparisons with the theoretical predictions. However, the information generated by the simulations goes well beyond those predictions: this is eminently true for the frequency spectra of the dynamical density-density and current-current correlation functions [dynamical structure functions $S(k, \omega), L(k, \omega), T(k, \omega)$ ]. Beyond predictions pertaining to the peak positions of the spectra, identified as the frequencies of the collective excitations, the QLCA does not provide, apart from some qualitative estimates, any basis for comparison in this respect. While other works, based mostly on the memory function formalism [51, 80, 81, 88], have presented theoretical descriptions of some of the features of the structure functions, we have made no attempt to relate to these, rather scant, results for the purpose of comparison with simulations.

As noted in Section 3.3, the basic hypotheses (i)-(iv) of the QLCA theory have undergone careful testing by a series of MD simulation experiments both for Coulomb and Yukawa systems and both for 2D and 3D configurations [34, 35]. With increasing $\Gamma$ values, a visual inspection of the potential landscape clearly indicates the formation of potential wells [35]. Examination of the phase space trajectories reveals a clear morphological difference between low $\Gamma$ and high $\Gamma$ situations: in the first case the trajectories are open, interrupted by propagating oscillatory portions, while in the second case the trajectories are mostly closed and exhibit a loop structure characteristic 
of localized oscillatory motion [34. An example of this behavior is illustrated for a 3D Coulomb liquid in figures $7(\mathrm{a})$ and (b) for $\Gamma=2.5$ and $\Gamma=160$, respectively.
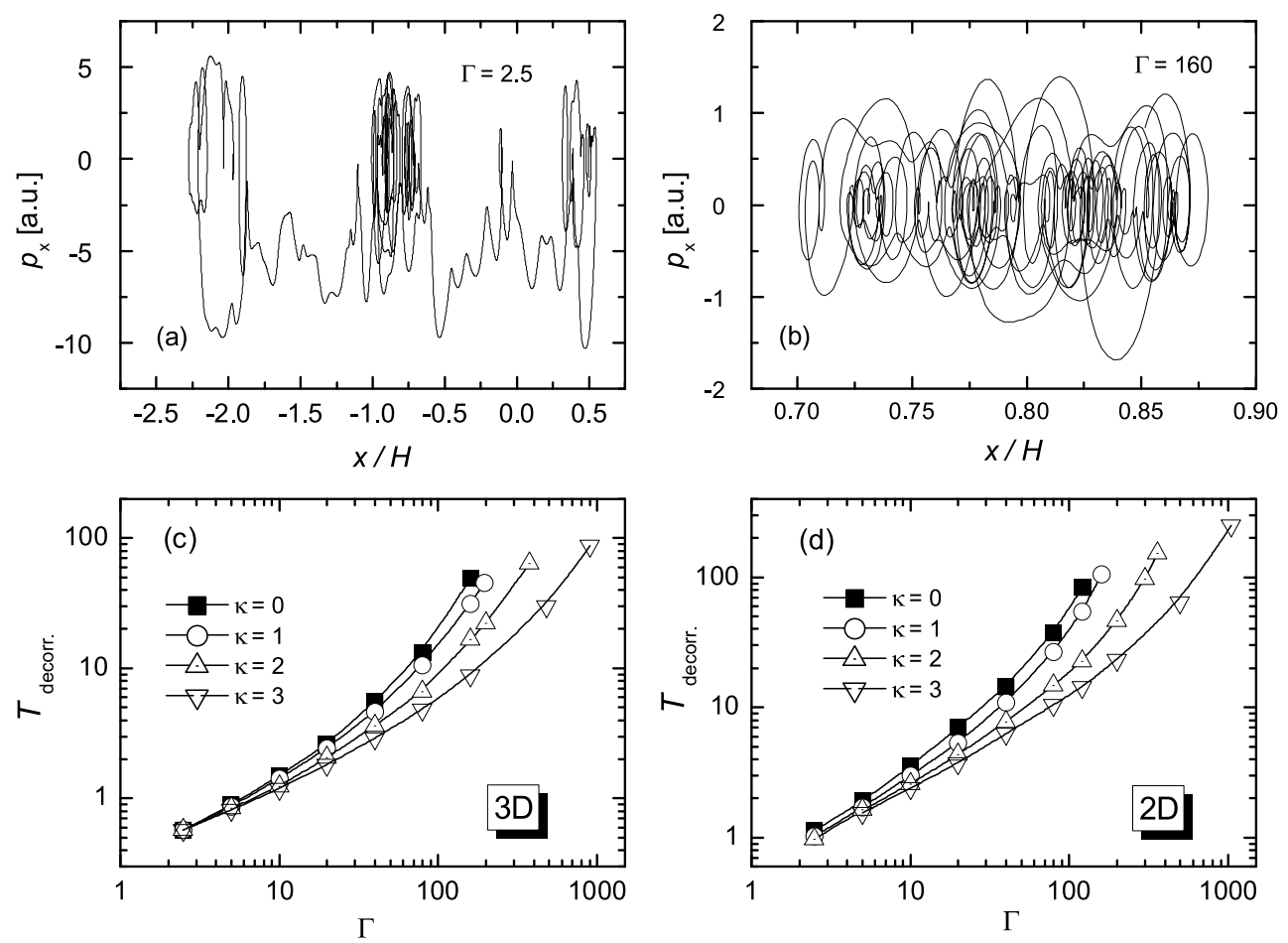

Figure 7. Phase space trajectory segments of a test particle in a 3D Coulomb liquid at (a) $\Gamma=2.5$ and (b) $\Gamma=160 . H$ is the edge length of the simulation box. Decorrelation time of the cages $\left(T_{\text {decorr }}=\omega_{0} t_{\text {decorr }} / 2 \pi\right)$ as a function of $\Gamma$ for the (c) $3 \mathrm{D}$ and (d) 2D systems, for a series of $\kappa$ values, obtained from MD simulations. (a,b) Reproduced from Ref. 34. Copyright (2002) by the American Physical Society. (c,d) Reprinted with permission from [Z. Donkó, P. Hartmann, and G. J. Kalman, Phys. Plasmas 10, (5), 1563 (2003)]. Copyright (2003) by the American Institute of Physics, Ref. 35].

The quantification of the relationship between localization and the strength of the coupling has been carried out by invoking a technique due to Rabani et al. [89]. Here a "cage correlation function" was introduced to characterize the gradual disintegration of the cage of the nearest neighbors and the escape of the caged particle. The main results shown in figures 7 (c) and (d) for 3D and 2D Coulomb and Yukawa systems illustrate the duration (in terms of plasma oscillation cycles) of the caging (decorrelation time, $\left.T_{\text {decorr }}\right)$ as a function of $\Gamma$ and $\kappa$. In the case of the $3 \mathrm{D}$ system, at $\kappa=0$ and $\Gamma=160$ the cages decorrelate during $\approx 50$ plasma oscillation cycles. The decorrelation time is reduced to a single cycle at $\Gamma \approx 7$. In the case of the $2 \mathrm{D}$ system it takes about 100 cycles for the cages to decorrelate at $\kappa=0$ and $\Gamma=120$, and we reach $T_{\text {decorr }}=1$ at $\Gamma \approx 2.5$. In the high- $\Gamma$ domain we observe a strong dependence of the decorrelation time on $\kappa$, both in $3 \mathrm{D}$ and $2 \mathrm{D}$ systems. At low values of $\Gamma$, however, $T_{\text {decorr }}$ depends only slightly on $\kappa$. The decrease of the decorrelation time for increasing $\kappa$ can be compensated by increasing $\Gamma$, as it can be seen in figure 7(c) and (d) [35. It is noted that the data 

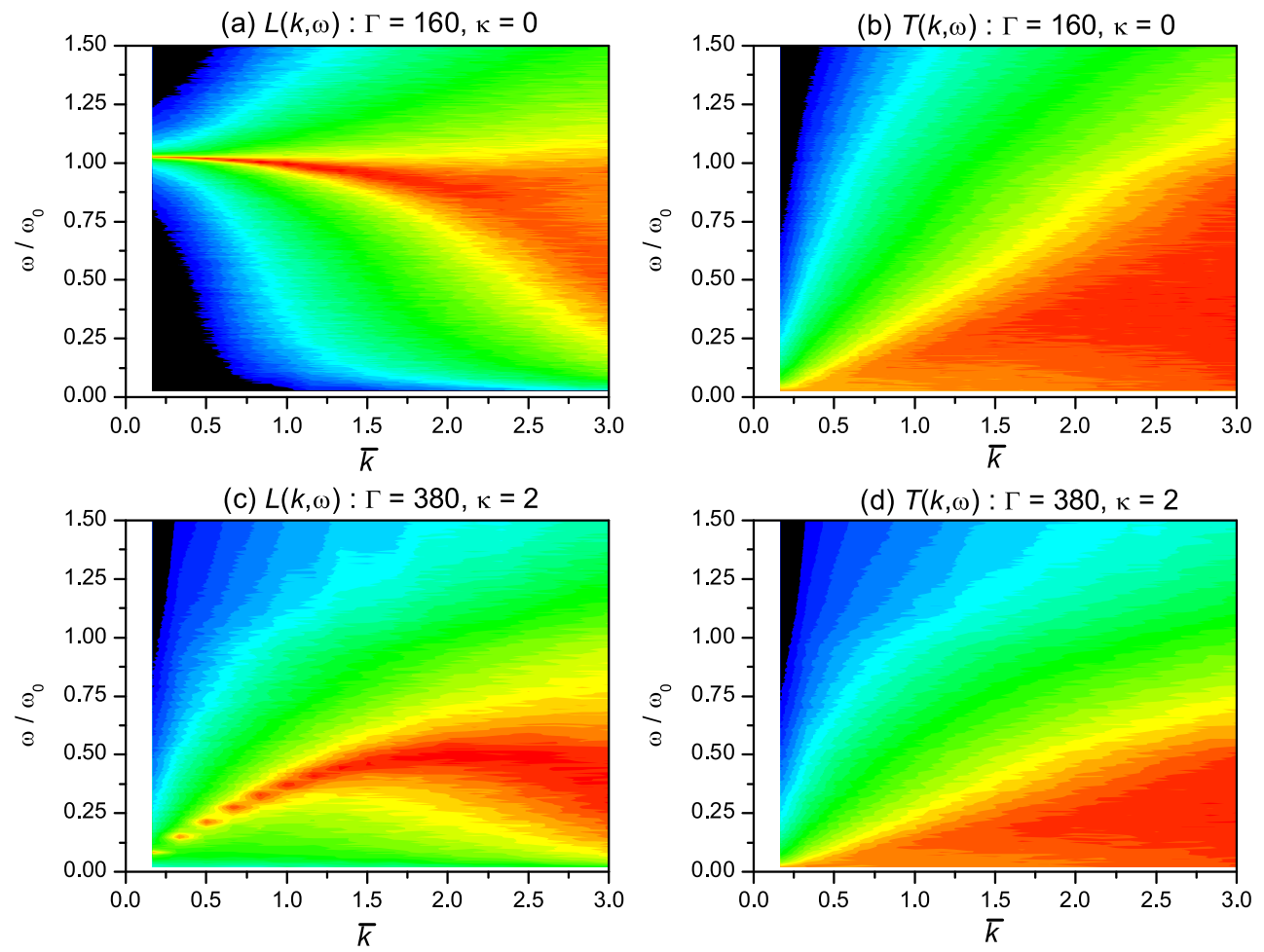

Figure 8. (Color online) 3D Yukawa and Coulomb liquids: spectral decomposition of the longitudinal and transverse current fluctuations in Coulomb $(\mathrm{a}, \mathrm{b})$ and Yukawa (c,d) plasmas, at $\Gamma=160, \kappa=0$, and $\Gamma=380, \kappa=2$, respectively. (The color coding of the amplitude is logarithmic, it only intends to illustrate qualitative features.)

shown in figure 7 convey information about the "average behavior" of the particles, it is however, recognized [35] that the surrounding of individual particles may change in a different way, due to e.g. avalanche type excitation and migration 90]. Finally we note that the caging of the particles at high $\Gamma$ values determines many of the systems properties as it has been discussed by Daligault for 3D Coulomb liquids [91.

\subsection{Three-dimensional Yukawa liquids}

The first molecular dynamics simulations on the wave dispersion relations in the fluid phase of 3D Yukawa systems were reported by Hamaguchi and Ohta [92, 93]. Their results confirmed the earlier theoretical predictions of Rosenberg and Kalman [72] on the longitudinal wave dispersion and were mostly in agreement with the simultaneously published full QLCA calculations of Kalman et al. [70]. They also demonstrated that the transverse wave dispersion has a cutoff at a long wavelength even in the case of weak screening.

This work was followed by a series of MD simulation for the collective excitations in 3D Yukawa liquids to provide further comparison with the predictions of the QLCA theory. The simulations - of which the results are presented here for the first time have been carried out using $N=12800-15625$ particles. 
To illustrate qualitatively the features of the behavior of the collectives excitations the spectral decomposition of the longitudinal and transverse current fluctuations is plotted in figure 8 for 3 -dimensional Coulomb and Yukawa liquids. In the case of the Coulomb plasma, at low wave numbers the frequency of the longitudinal $(\mathcal{L})$ mode is concentrated within a narrow frequency range [see figure 8(a)] near the plasma frequency. With increasing wave number the frequency of the mode gradually spreads over a wider domain and shows a slightly decreasing tendency. In sharp contrast with this behavior the $\mathcal{T}$ mode frequency is spread over a wide domain, as illustrated in figure $\mathbb{8}$ (b). The $\mathcal{L}$ mode of the Yukawa system is quite different from that in the Coulomb case, the wave frequency approaches zero at $\bar{k} \rightarrow 0$ wave number. The frequency increases with increasing wave number up to about $\bar{k}=2.0$, and then starts to decrease slightly. Meanwhile the frequency distribution gets gradually wider. The $\mathcal{T}$ mode in the Yukawa case appears to be similar to the corresponding mode in the Coulomb system, although the frequency is lower, due to the weaker interaction of the particles, as a consequence of the screened potential.

For a better quantitative analysis representative dynamical structure functions (density fluctuation spectra) $S(k, \omega)$ and spectra of the longitudinal and transverse current fluctuations, $L(k, \omega)$ and $T(k, \omega)$, are plotted in figures 9 and 10, respectively, for a high- $\Gamma$ and a medium- $\Gamma$ case. The $S(k, \omega)$ obtained for the Coulomb case $(\Gamma=$ $160, \kappa=0$, see figure 9(a)) peaks at nearly the same frequency for the different values of the wave numbers plotted, which are multiples of $\bar{k}_{\min }=0.167$ (determined by the size of the simulation box). In the presence of screening (Yukawa potential), as shown in figure 9 (d), the behavior of $S(k, \omega)$ changes significantly: at $\bar{k} \rightarrow 0$ the wave frequency $\omega / \omega_{0} \rightarrow 0\left[\omega_{0}\right.$ is defined by (6) $]$. The contrast between the $\kappa=0$ and the $\kappa>0$ cases is also well seen in figure 11(a), where the dispersion curves derived from the fluctuation spectra are displayed. The dispersion curves for $\kappa>0$ are quasi-acoustic $\left(\omega / \omega_{0} \propto \bar{k}^{1 / 2}\right)$, with a linear portion near $k=0$, which gradually extends when $\kappa$ is increased. The $(\Gamma, \kappa)$ pairs for which the dispersion graphs are plotted in figure 11 have been selected to represent a constant effective coupling $\Gamma^{*}=160$. This definition of $\Gamma^{*}$ relies on the constancy of the first peak amplitude of the pair correlation function $g(\bar{r})$, similarly to the case of 2D Yukawa liquids [32].

Peaks in the spectra of the compressional $\mathcal{L}$ mode [plotted in panels (b) and (e) of figures 9 and [10] appear at the same frequency as those in the corresponding $S(k, \omega)$ functions, as these functions are linked via the relation

$$
L(k, \omega)=\frac{\omega^{2}}{k^{2}} S(k, \omega) .
$$

Compared to those characterizing the $\mathcal{L}$ mode, peaks in the $\mathcal{T}$ mode spectra are rather broad, as it can be seen in panels (c) and (f) of figures 9 and 10. In the case of this mode there is no dramatic change between the behavior when $\kappa$ changes from zero to a nonzero value, only the mode frequency decreases, as can be observed in figure 11(b).

Comparison of the dispersion relations obtained from the MD simulations [via $S(k, \omega)$ ] and QLCA calculations [see equations (33)-(35)] is presented in figure 11. Here, 

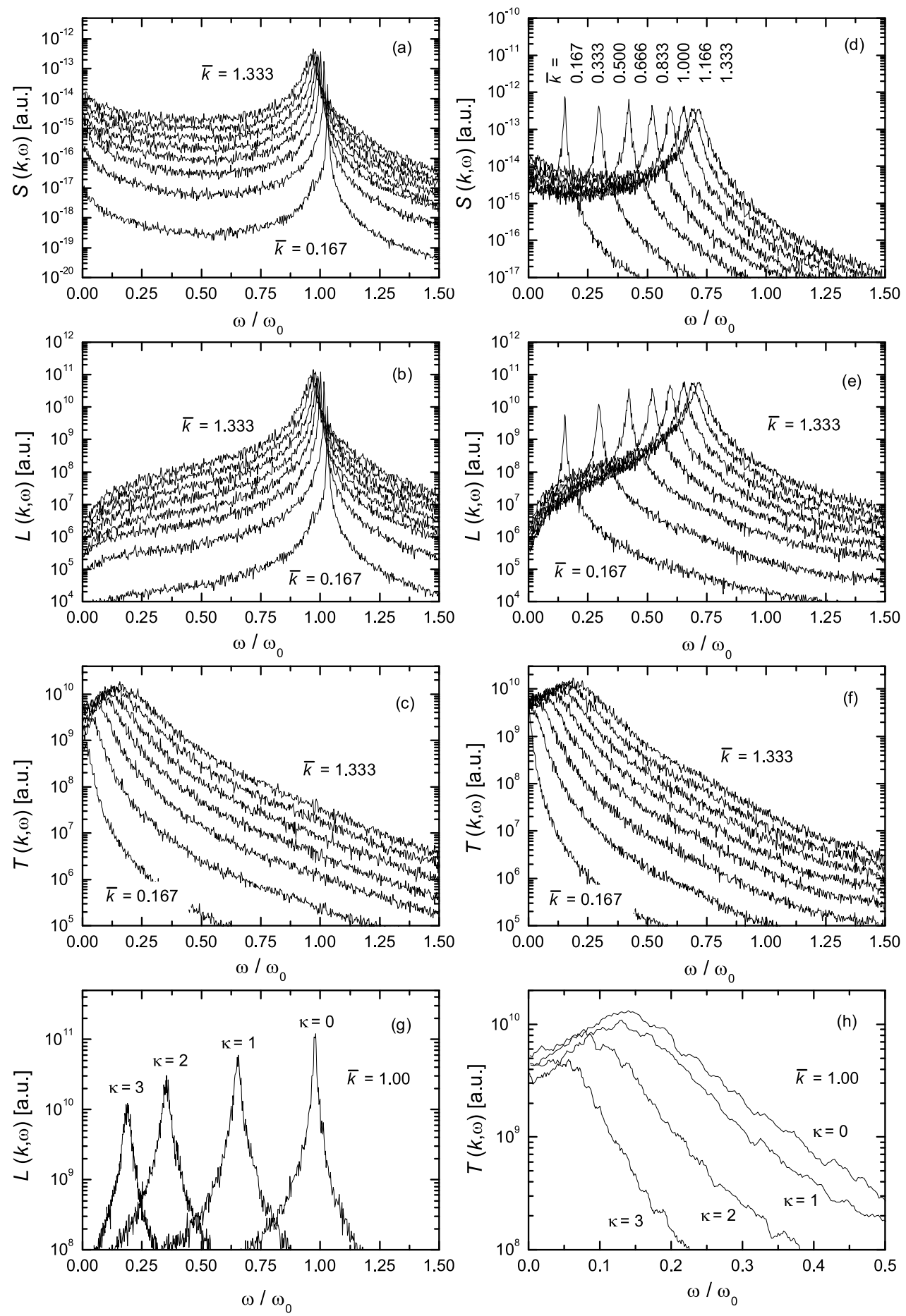

Figure 9. 3D Yukawa and Coulomb liquids: density $[S(k, \omega)]$ and current $[L(k, \omega)$ and $T(k, \omega)]$ fluctuation spectra of Coulomb $\Gamma=160, \kappa=0(\mathrm{a}, \mathrm{b}, \mathrm{c})$ and Yukawa $\Gamma=200$, $\kappa=1$ (d,e,f) systems. The curves are plotted for multiples of the smallest accessible wave number $\bar{k}_{\min }=0.167$. (g) and (h) show the dependence of $L(k, \omega)$ and $T(k, \omega)$, respectively, on $\kappa$ at fixed wave number $\bar{k}=1.00$. ( $\Gamma=360$ for $\kappa=2$, and $\Gamma=1050$ for $\kappa=3)$. 

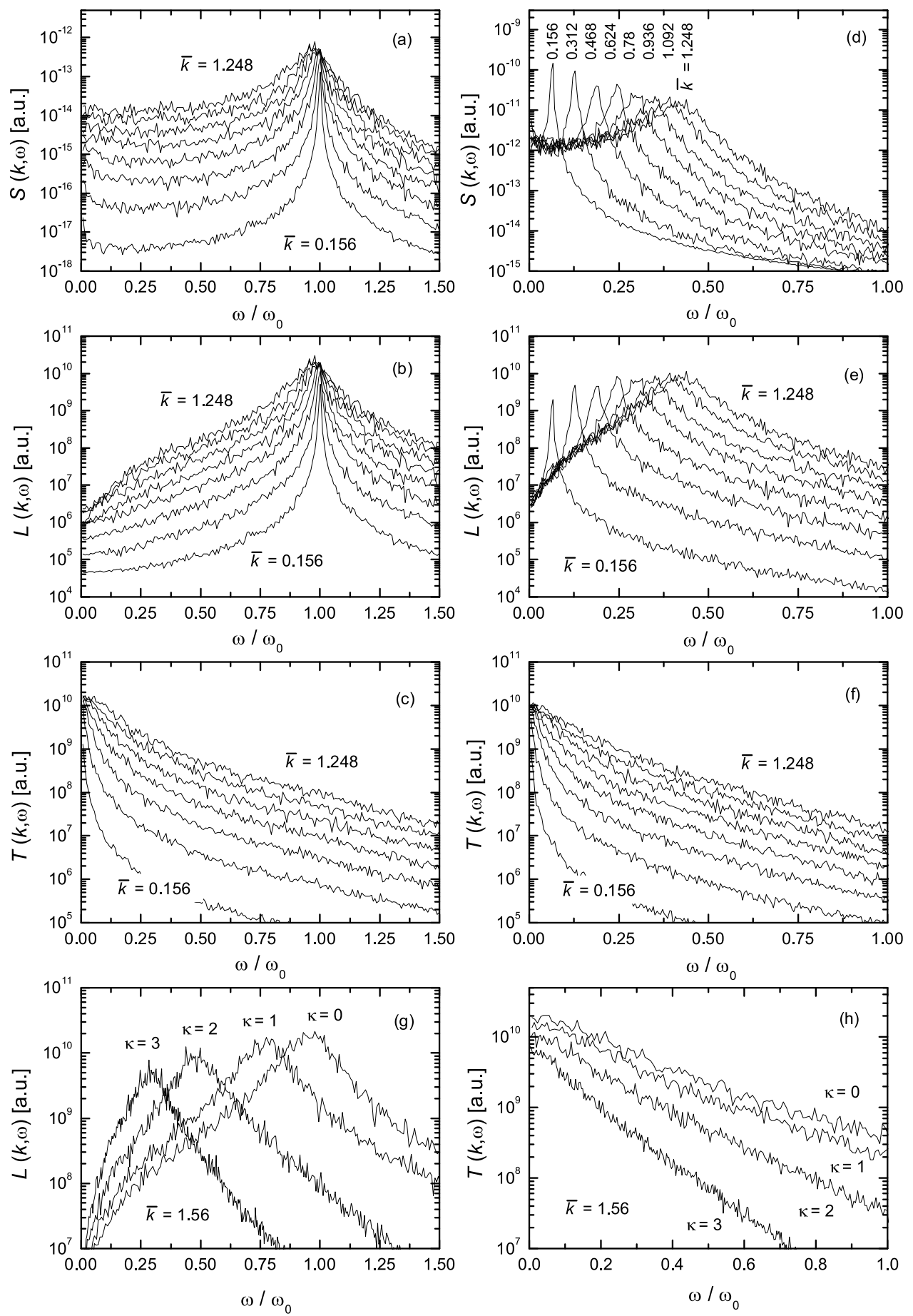

Figure 10. 3D Yukawa and Coulomb liquids: density $[S(k, \omega)]$ and current $[L(k, \omega)$ and $T(k, \omega)]$ fluctuation spectra of Coulomb $\Gamma=20, \kappa=0$ (a,b,c) and Yukawa $\Gamma=48$, $\kappa=2(\mathrm{~d}, \mathrm{e}, \mathrm{f})$ systems. The curves are plotted for multiples of the smallest accessible wave number $\bar{k}_{\min }=0.156$. (g) and (h) show the dependence of $L(k, \omega)$ and $T(k, \omega)$, respectively, on $\kappa$, at fixed wave number $\bar{k}=1.56$. $(\Gamma=25$ for $\kappa=1$, and $\Gamma=114$ for $\kappa=3)$. 

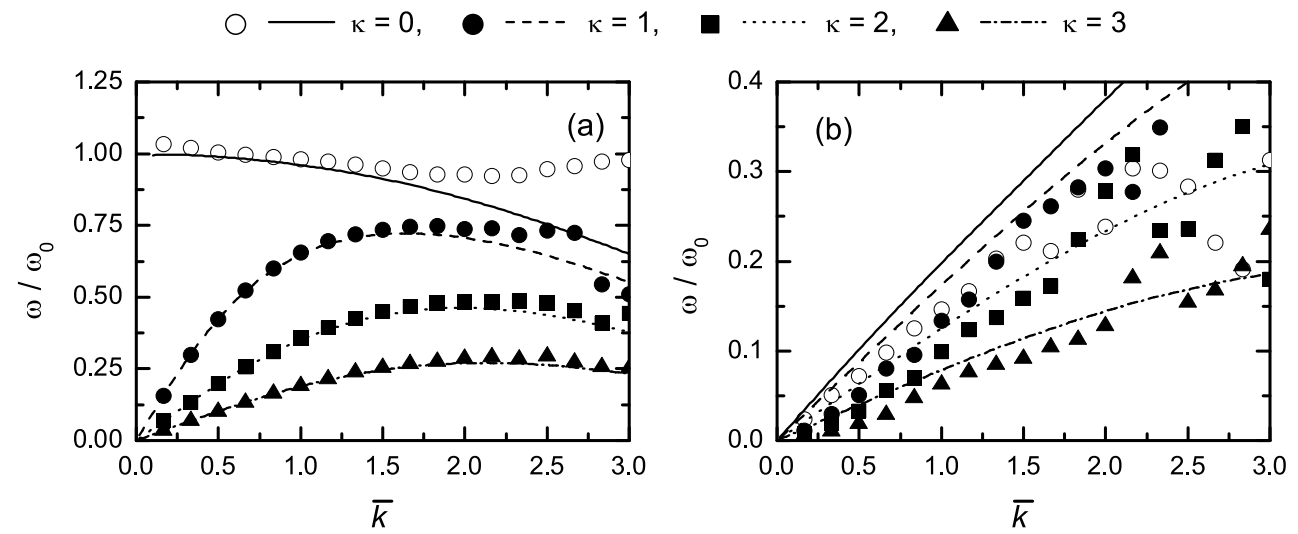

Figure 11. 3D Yukawa and Coulomb liquids: dispersion relations for the (a) longitudinal and (b) transverse modes. $O: \Gamma=160, \kappa=0$ (Coulomb case),

$: \Gamma=200, \kappa=1, \boldsymbol{\square}: \Gamma=380, \kappa=2$, and $\boldsymbol{\Delta}: \Gamma=910, \kappa=3$. Symbols represent molecular dynamics results, while the lines correspond to the predictions of the QLCA theory.
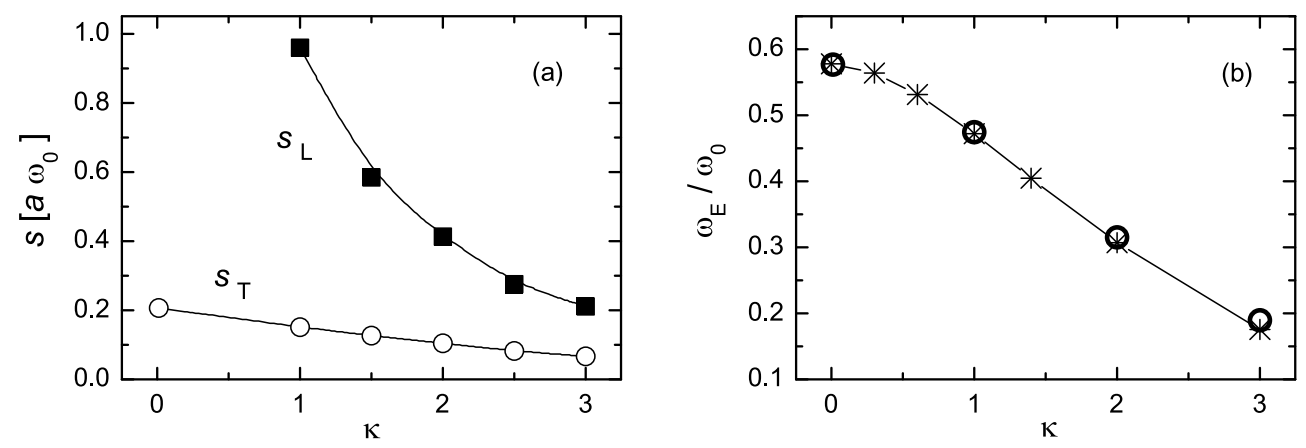

Figure 12. 3D Yukawa and Coulomb liquids: (a) sound velocities and (b) Einstein frequency as derived from the QLCA theory (circles) and Einstein frequencies of fcc lattice (stars) 94.

in the calculations of the QLCA results, we have made use of the $g(r)$ functions obtained from the MD simulation. The agreement between the two sets of data is excellent for the $\mathcal{L}$ mode, while some difference in the frequency of the $\mathcal{T}$ waves can be seen in figure 11(b). This latter may originate from the inaccurate determination of the peak positions of the rather broad $T(k, \omega)$ spectra. It should be noted though that while the theoretical calculations provide an oscillatory dispersion curve for $\bar{k}>3$ (see figure 2), simulations provide reliable results (for collective excitations) for typical liquid-phase conditions for $\bar{k} \lesssim 3$. (At higher $\bar{k}$ values the thermal contribution in $S(k, \omega)$ apparently masks the collective mode peak.). The simulation results here resemble the measured 2D dispersion curves in the liquid phase [15]. Another difference is the cutoff of the $\mathcal{T}$ mode dispersion curve at finite wave numbers. This disappearance of the shear modes for $\bar{k} \rightarrow 0$ is a well known feature of the liquid state [50, 95, 96], and the sharp cut-off $\omega \rightarrow 0$ for a finite $k$ has also been observed in simulations of Yukawa systems [53, 93]. 
It has been already noted that this cutoff is not accounted for by the QLCA, as it does not include damping effects.

The sound velocities, derived in (37), are plotted in figure 12(a), while figure 12(b) displays the Einstein frequency, which is defined in (36). In the $\kappa \rightarrow 0$ limit (36) gives $\omega_{\mathrm{E}}=\omega_{0} / \sqrt{3}$, and $\omega_{\mathrm{E}}$ decreases with increasing $\kappa$. For comparison, the Einstein frequency data of Ohta and Hamaguchi for a fcc lattice [94] are also plotted in figure 12(b). We find an excellent agreement between the two sets of data.
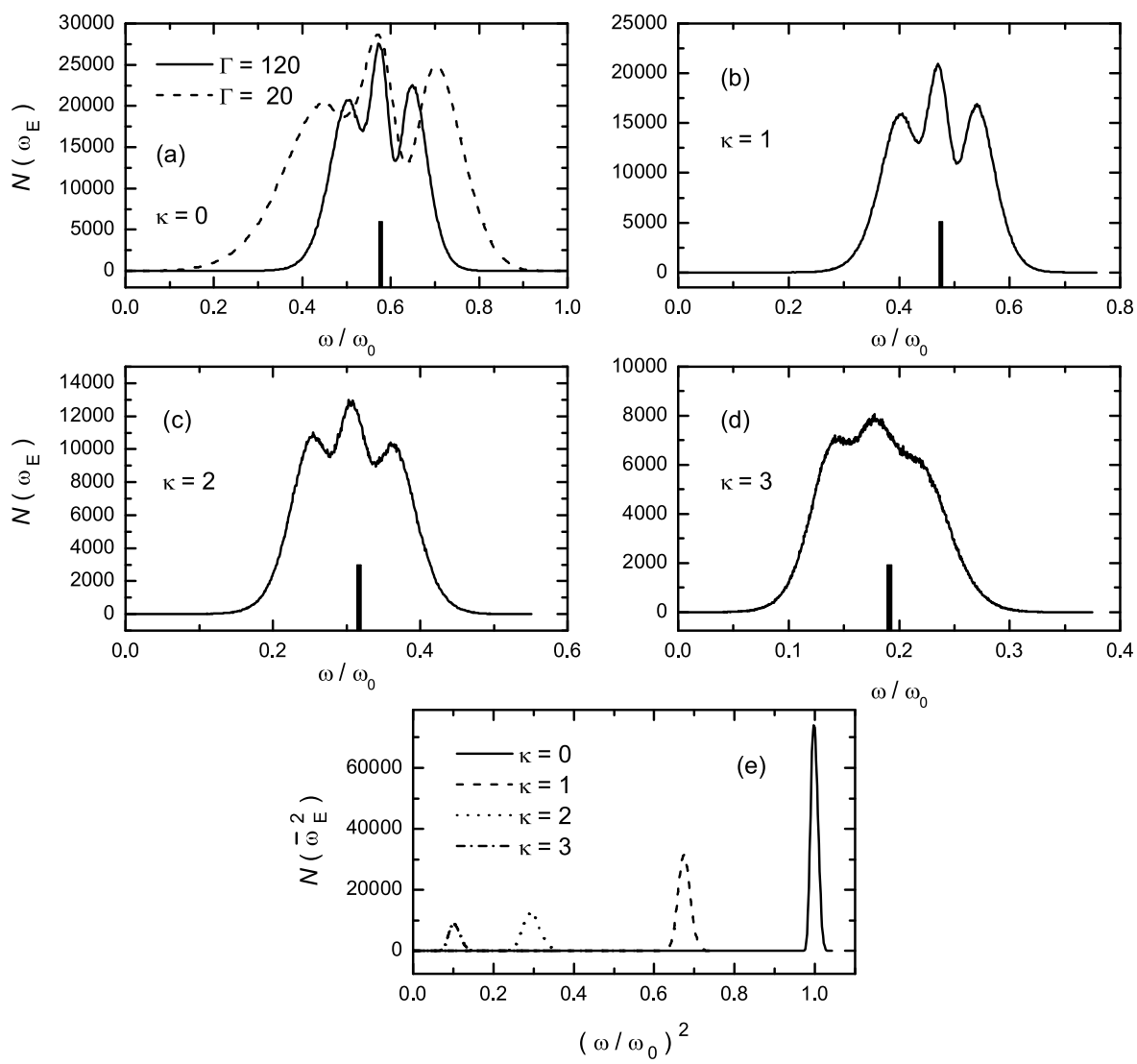

Figure 13. 3D Yukawa and Coulomb liquids: Einstein frequency distributions for $\Gamma^{\star}=120$ : (a) $\Gamma=120, \kappa=0$; (b) $\Gamma=150, \kappa=1$; (c) $\Gamma=300, \kappa=2$; (d) $\Gamma=725$, $\kappa=3$; the vertical bars indicate values obtained by the QLCA theory. (a) also shows the distribution of frequencies at the lower coupling value $\Gamma=20$. (e) Distribution of $\bar{\omega}_{\mathrm{E}}^{2}$ for the same systems.

Numerical experiments were also performed to determine the distribution of the microscopic Einstein frequency $\omega_{\mathrm{E}}$. To accomplish this, frequency histograms based on a few hundred, temporally uncorrelated particle configurations have been constructed. For the raw (particle position) data the harmonic matrix for every particle has to be generated:

$$
H_{\alpha \beta}^{(i)}=\sum_{j \neq i}^{N} \frac{\partial^{2} \phi\left(\left|\mathbf{r}_{i}^{\mathrm{eq}}-\mathbf{r}_{j}\right|\right)}{\partial r_{i, \alpha} \partial r_{i, \beta}}
$$


where $\mathbf{r}_{i}^{\mathrm{eq}}$ is the equilibrium position of the $i$-th particle (local minimum of the potential surface), $\phi(r)$ is the interaction potential, $\alpha$ and $\beta$ represent the Cartesian coordinates. The eigenvalues of $H_{\alpha \beta} / m$ are the squared Einstein frequencies (3 for every particle), while the eigenvectors provide the polarization of the oscillation.

A series of frequency histograms for an effective coupling parameter $\Gamma^{\star}=120$ and different values of $\kappa$ are shown in figures 13(a)-(d). The frequency distributions exhibit three peaks (although this is less visible in the $\kappa=3$ case). With increasing screening the distribution of frequencies becomes wider and its mean value is shifted towards lower frequency. The QLCA results for the Einstein frequency [obtained from Eq.(36) using pair correlation functions generated in the MD simulation], corresponding to the different values of $\kappa$ are also indicated in figures 13(a)-(d). The values are in good agreement with the simulation results. The effect of $\Gamma$ at fixed $(\kappa=0)$ screening is illustrated in figure 13(a). A six time decrease of the coupling parameter results in approximately doubled width of the Einstein frequency distribution.

Figure 13(e) shows the histograms for $\bar{\omega}_{\mathrm{E}}^{2}$, sums of the 3 microscopic squared Einstein frequencies, for different values of $\kappa$ : there is a qualitative difference between the $\kappa=0$ Coulomb case where there is only a single frequency (a narrow peak) and the $\kappa>0$ cases, where a distribution of frequencies is apparent. The reason for this difference has been discussed in Section 3 ,

Further information on the collective behavior is contained in the velocity autocorrelation function (VACF)

$$
Z(t)=\frac{\langle\mathbf{v}(t) \mathbf{v}(0)\rangle}{\left\langle|\mathbf{v}(0)|^{2}\right\rangle},
$$

where the average is taken over the $N$ particles and different initial times.

The behavior of the velocity autocorrelation functions of 3D Yukawa liquids obtained at several values of the $\Gamma$ and $\kappa$ parameters is illustrated in figure 14, Analyzing the behavior of $Z(t)$ at constant $\kappa$, we find a transition from monotonically decreasing $Z(t)$ into an oscillating type when $\Gamma$ is increased [figure 14(a)]. Similarly, the shape of $Z(t)$ changes drastically when $\kappa$ is varied at constant $\Gamma$, as shown in figure 14(b). For more detailed analysis see [94].

Using the Einstein frequency $\omega_{\mathrm{E}}$ for the normalization of time, instead of the plasma frequency $\omega_{0}$ (as in figure 14) the $Z(t)$ functions belonging to the same $\Gamma^{\star}=160$ for a series of $\kappa$ values are displayed in figure 15(a). Using this normalization of the timescale the $Z(t)$ curves exhibit a nearly universal behavior, where at least the first few peaks of $Z(t)$ nearly overlap. This observation emphasizes the importance of the Einstein frequency in the dynamical behavior of the system.

The marked oscillations of the $Z(t)$ function in the Coulomb case is indeed expected on the basis of the possible coupling between the single particle motion and longwavelength plasmons, whose frequencies are almost independent of $k$. For $\kappa>0$, however, even though $\omega(k \rightarrow 0) \propto k$, the oscillations persist; this can be explained by the fact that the $\omega(k)$ dispersion curve flattens at higher wave numbers, a corresponding peak in the frequency distribution develops. 

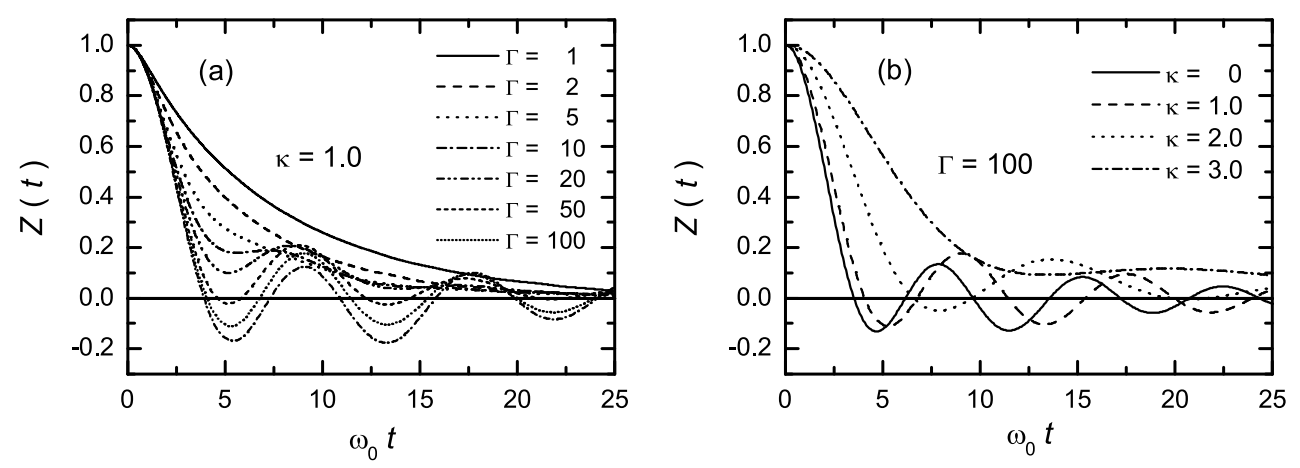

Figure 14. 3D Yukawa and Coulomb liquids: (a) velocity autocorrelation functions at $\kappa=1.0$ and a series of $\Gamma$ values; (b) at constant $\Gamma=100$, for a series of $\kappa$ values.
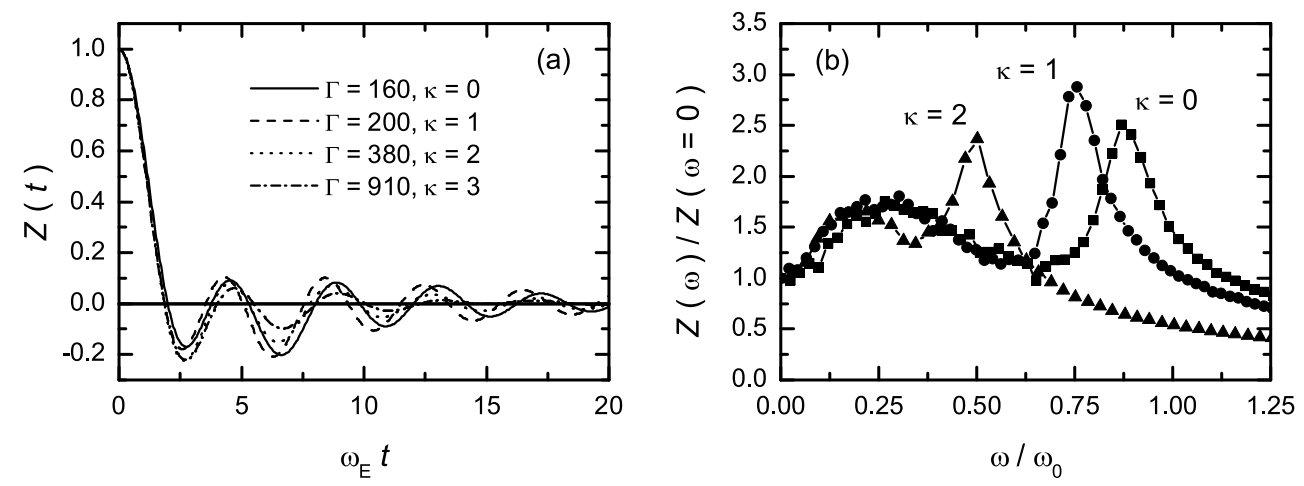

Figure 15. 3D Yukawa and Coulomb liquids: (a) velocity autocorrelation functions for a series of $\kappa$ values; the time is normalized by the Einstein frequency $\omega_{\mathrm{E}}$. (b) corresponding Fourier transforms $Z(\omega)$, for $(\Gamma, \kappa)$ pairs as indicated in (a).

The Fourier transforms $Z(\omega)$ of the VACF functions (obtained at different $\kappa$ values but for constant effective coupling $\Gamma^{\star}=160$ ) are portrayed in figure 15(b). The dominant peaks in the spectra - shifting towards lower frequencies with increasing $\kappa$ - correspond to the high frequency oscillations of the $Z(t)$ functions (easily observed visually). As discussed in previous studies (see e.g. [94, 96]) these peaks are related to longitudinal current fluctuations, while the broad features at low frequencies are connected to the transverse current fluctuations and are related to diffusion properties of the system. Taking the case of $\kappa=2$ as an example, figure 8 (c) indicates that most of the energy of the $\mathcal{L}$ mode is concentrated around frequencies $\omega / \omega_{0} \cong 0.5$, in correspondence with the peak of the $Z(\omega)$ function shown in figure 15(b). The $T(\bar{k}, \omega)$ spectra [see figure 8 (c)] for any $\bar{k}$ are broader, compared to the $L(\bar{k}, \omega)$ spectra: the fluctuations in the transverse currents are distributed over a rather broad frequency domain, again in agreement with the behavior of the corresponding $Z(\omega)$ function. The observed features of $Z(\omega)$ indicate an appreciable coupling between single particle motion and collective excitations in the $2 \mathrm{D}$ system.

In addition to the peaks in the frequency spectrum of the dynamical structure 

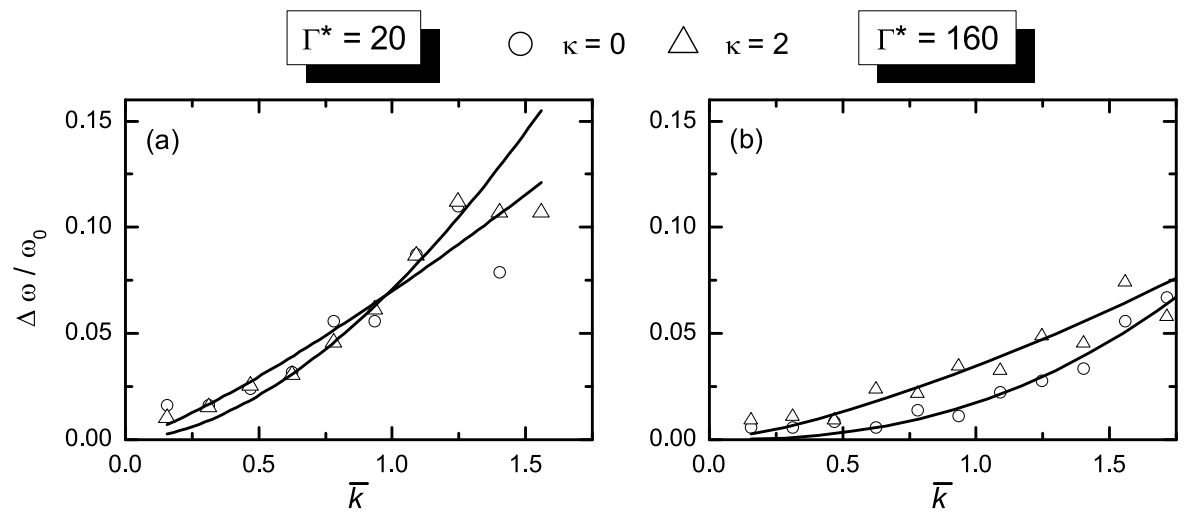

S]
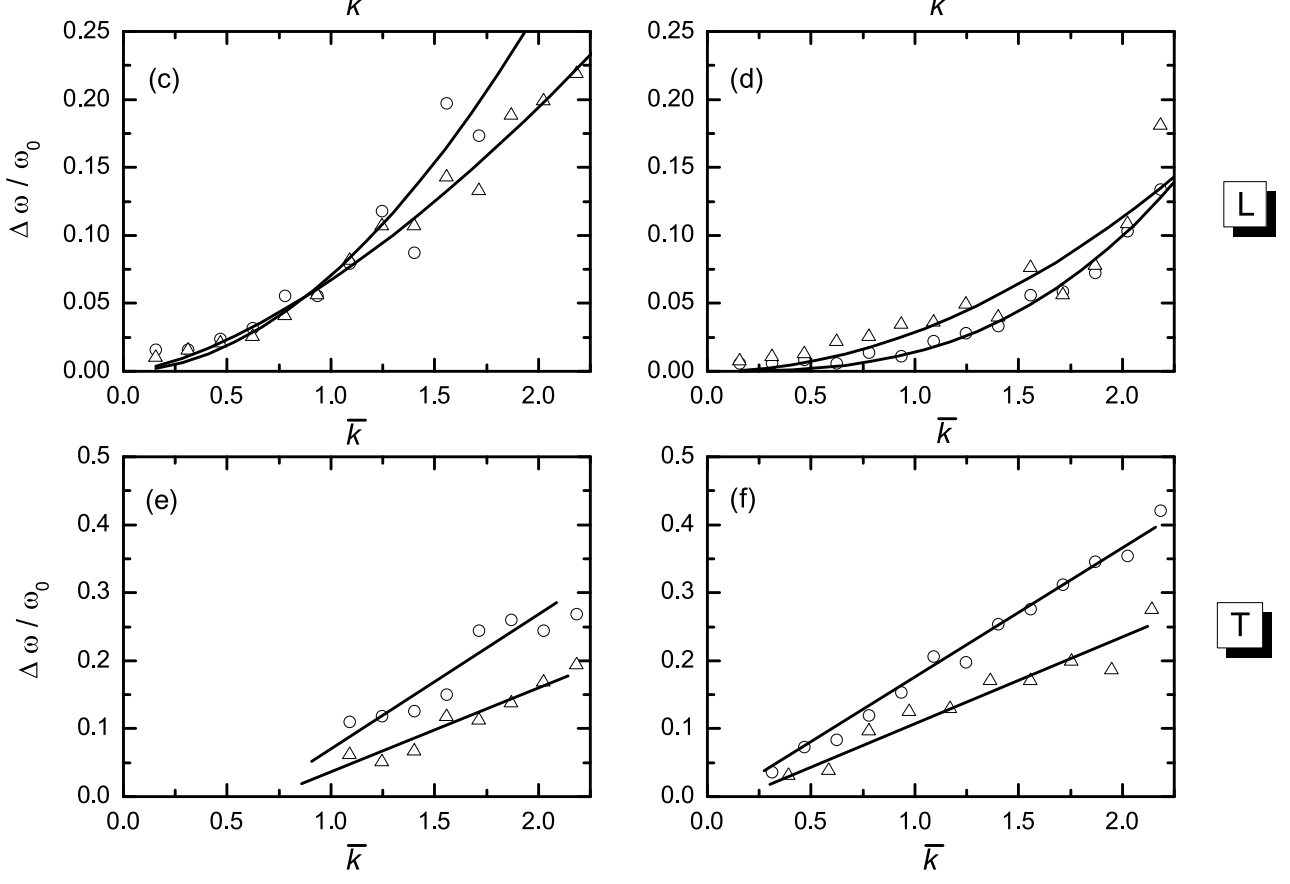

Figure 16. 3D Yukawa and Coulomb liquids: spectral line width of functions $S(k, \omega)$ $(\mathrm{a}, \mathrm{b}), L(k, \omega)(\mathrm{c}, \mathrm{d})$ and $T(k, \omega)(\mathrm{e}, \mathrm{f})$ for $\Gamma^{\star}=20$ and $\Gamma^{\star}=160$ effective coupling at $\kappa=0$ and $\kappa=2$.

functions a wealth of further physical information is contained in the detailed structures of these quantities. Of great importance would be the understanding of the evolution of the width of the frequency spectra as functions of $k$ and $\Gamma$, since it is related to the damping of the collective modes. To illustrate the behavior of the dynamical structure function figure 16 shows the widths of the collective mode peaks as a function of wave number, for effective coupling values $\Gamma^{\star}=20$ and 120 , for $\kappa=0$ and 2 . It is noted that Murillo has provided a formula for the width of the transverse current spectrum [53]. 


\subsection{Two-Dimensional Yukawa liquids}

Most of the available experimental evidence on waves in complex plasmas relates to 2D systems (see section 5); much less information can be culled from observations on 3D systems. Thus the understanding of the collective mode structure in the different phases of the 2D Yukawa system has been of great current interest: over the past few years a substantial amount of simulation work has been performed on 2D Yukawa liquids [71, 97]. In the following we present these MD simulation results on the dispersion properties of the liquid state and compare them with the theoretical predictions of the QLCA analysis of the collective modes.

Representative density fluctuation spectra, as well as longitudinal and transverse current fluctuation spectra of the 2D Yukawa liquid are displayed in figure 17. The dispersion curves derived from the simulation spectra $S(k, \omega)$ for both modes are displayed in figure 18. The results shown in the latter figure at $\kappa=0$ reproduce the known 2D Coulomb dispersion [95, 98. With increasing $\kappa$ the mode frequencies rapidly diminish and the dispersion deviates more substantially from its RPA value. In the $k \rightarrow 0$ limit both modes exhibit an acoustic behavior, with longitudinal and transverse sound velocities $s_{\mathrm{L}}$ and $s_{\mathrm{T}}$ [68, 98, see Eq. (32). For the longitudinal mode the simulation data well corroborate the theoretical predictions with the proviso already noted in relation to the 3D liquid: that while the theoretical calculations provide an oscillatory dispersion curve for $\bar{k}>3$ (see figure 11), simulations provide reliable results (for collective excitations) for the given conditions for $\bar{k} \lesssim 3$. In the case of the transverse mode, the agreement between theory and MD data for moderately high $k$ values is fairly good; for $k \rightarrow 0$ the agreement is marred by the QLCA's inability to account for diffusional and other damping effects [98] that preclude the existence of long wavelength shear waves in the liquid state. As a result of this damping, a cutoff at a finite $k_{\mathrm{c}}$ and zero frequency develops (a similar phenomenon was observed in the $3 \mathrm{D}$ case [53, 93]). The $k_{\mathrm{c}}$ value is related to the diffusional-migrational time [98] through $\tau_{\mathrm{DM}}=1 / k_{\mathrm{c}} s_{\mathrm{T}}$, where $s_{\mathrm{T}}$ is the transverse sound velocity. Incorporating $\tau_{\mathrm{DM}}$, calculated with the aid of the theoretically predicted $s_{\mathrm{T}}$ values, in the QLCA equations as a phenomenological damping $\nu=1 / \tau_{\mathrm{DM}}$ (by the $\omega \rightarrow \omega+i \nu$ replacement), good agreement between the theory and the MD data was restored [71]. The simulations show that the longitudinal mode is not affected by this damping mechanism: this may indicate that its characteristic damping time is substantially longer.

The sound velocities and the Einstein frequency - given as the $k \rightarrow \infty$ limit of

Eqs. (26) or (28) - are shown in figure 19. For comparison, also displayed is the thermodynamic sound velocity generated from the equation of state of a Yukawa liquid [32. The sound velocities obtained here are extremely close to those of the hexagonal crystal [80]. The Einstein frequency diminishes rapidly with increasing $\kappa$, similarly to the $3 \mathrm{D}$ case $[70,94]$.

It is of interest to follow the evolution of the mode structure across the liquidsolid phase boundary, as the isotropic liquid dispersion transits into the anisotropic 

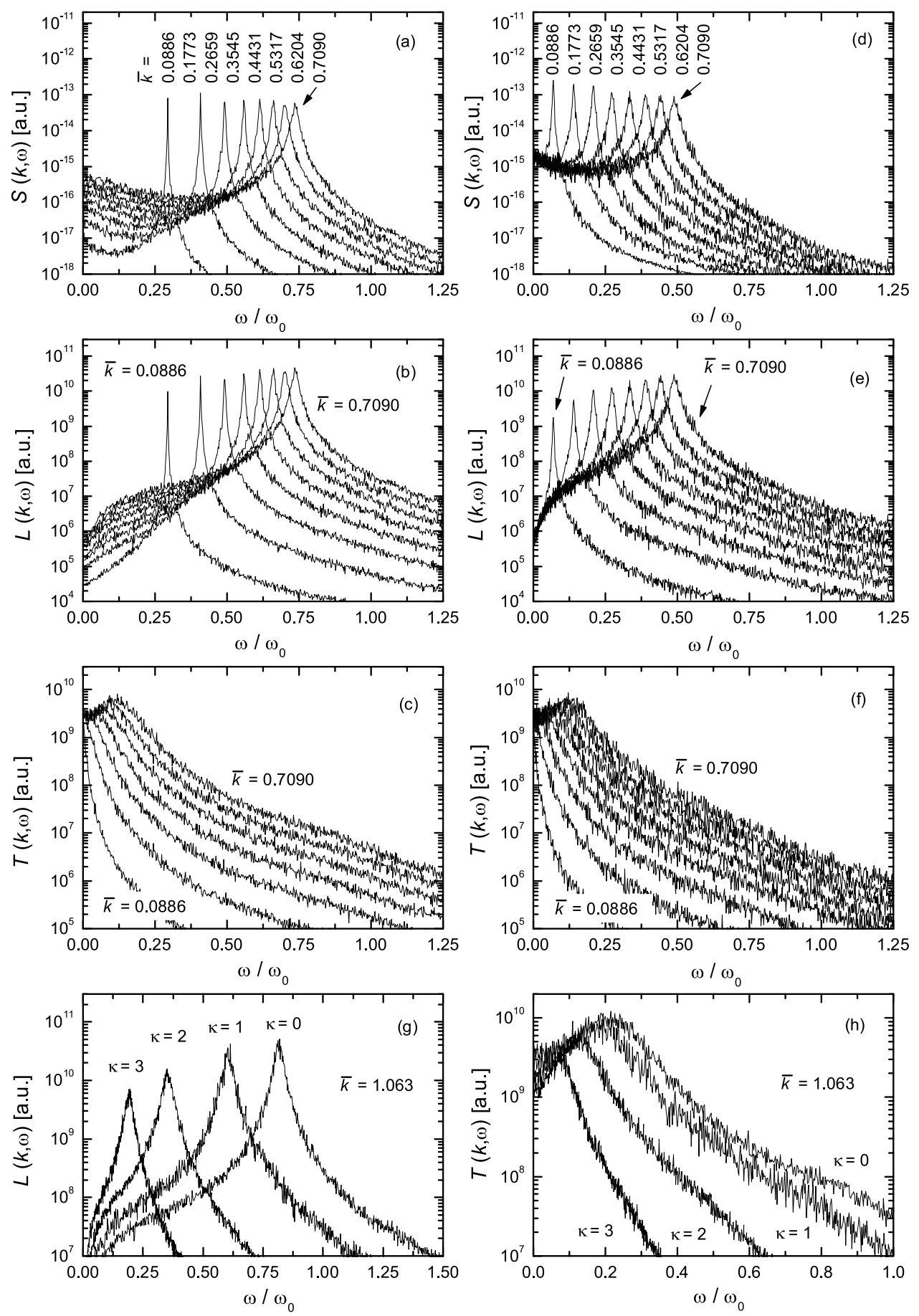

Figure 17. 2D Yukawa and Coulomb liquids: density $[S(k, \omega)]$ and current $[L(k, \omega)$ and $T(k, \omega)$ ] fluctuation spectra of Coulomb $\Gamma=120, \kappa=0(\mathrm{a}, \mathrm{b}, \mathrm{c})$ and Yukawa $\Gamma$ $=160, \kappa=1(\mathrm{~d}, \mathrm{e}, \mathrm{f})$ systems. The curves are plotted for multiples of the smallest accessible wave number $\bar{k}_{\text {min }}=0.0886$. (g) and (h) show the dependence of $L(k, \omega)$ and $T(k, \omega)$, respectively, on $\kappa$, at fixed wave number $\bar{k}=1.063$. ( $\Gamma=360$ for $\kappa=2$, and $\Gamma=1050$ for $\kappa=3$ ). 

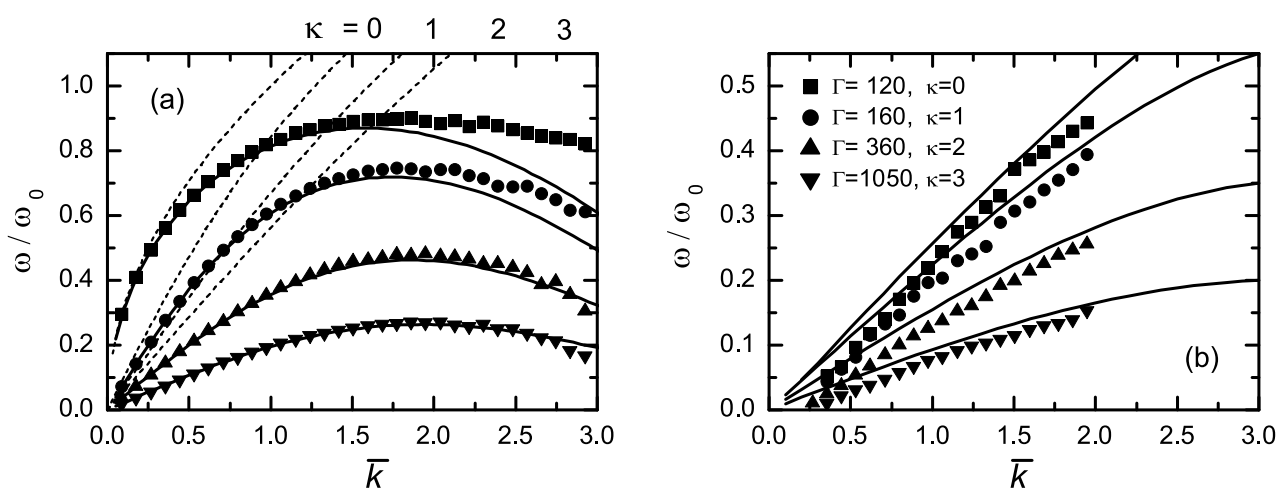

Figure 18. 2D Yukawa and Coulomb liquids: dispersion curves for (a) the longitudinal $(\mathcal{L})$ and $(\mathrm{b})$ transverse $(\mathcal{T})$ modes at $\Gamma^{\star}=120$ and $\kappa=0,1,2$, 3. Continuous curves: QLCA calculations; symbols: MD simulation; dashed lines: RPA dispersions. Reproduced from Ref. 71]. Copyright (2004) by the American Physical Society.
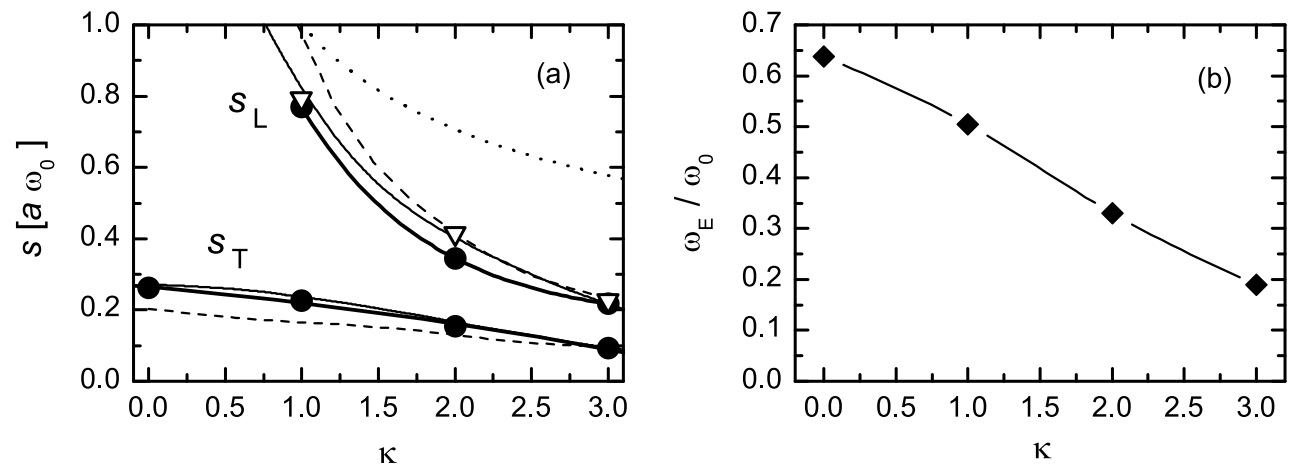

Figure 19. 2D Yukawa and Coulomb liquids: (a) sound velocities (heavy lines with circles: calculated from QLCA at $\Gamma^{\star}=120$, thin lines: hexagonal crystal lattice [80, dotted line: RPA values for $s_{\mathrm{L}}$, and dashed lines: $3 \mathrm{D}$ values at $\Gamma=160$, open triangles: thermodynamic sound velocity) [70, 71]; (b) calculated Einstein frequency as obtained from the QLCA formula for $\Gamma^{\star}=120$. Reproduced from Ref. [71. Copyright (2004) by the American Physical Society.

dispersion of the solid state. This is illustrated for the $\kappa=2$ case in figure 20, $\Gamma=500$ represents a relatively high temperature solid, where lattice defects may already show up, but the overall behavior (sharp separation of the mode frequencies along the $x$ and $y$ directions; compare e.g. the curves labeled Tx and Ty) reflects the conservation of the triangular crystalline structure. The $\Gamma=405$ case corresponds to a temperature slightly higher than the melting temperature (our results indicate that the transition occurs at $\Gamma \cong 415$ for $\kappa=2$ [82]), where all long range order in the system has already been extinguished, but locally most of the particles sit in the somewhat distorted hexagonal environment. The "oscillatory" feature in the $T$ mode around $\bar{k}=2.5$ can be taken as an indication for the transition from the ordered lattice to the disordered liquid state through the formation of disoriented domains of local hexagonal order. The orientation of these domains becomes more uncorrelated with increasing temperature. The $\Gamma=200$ 

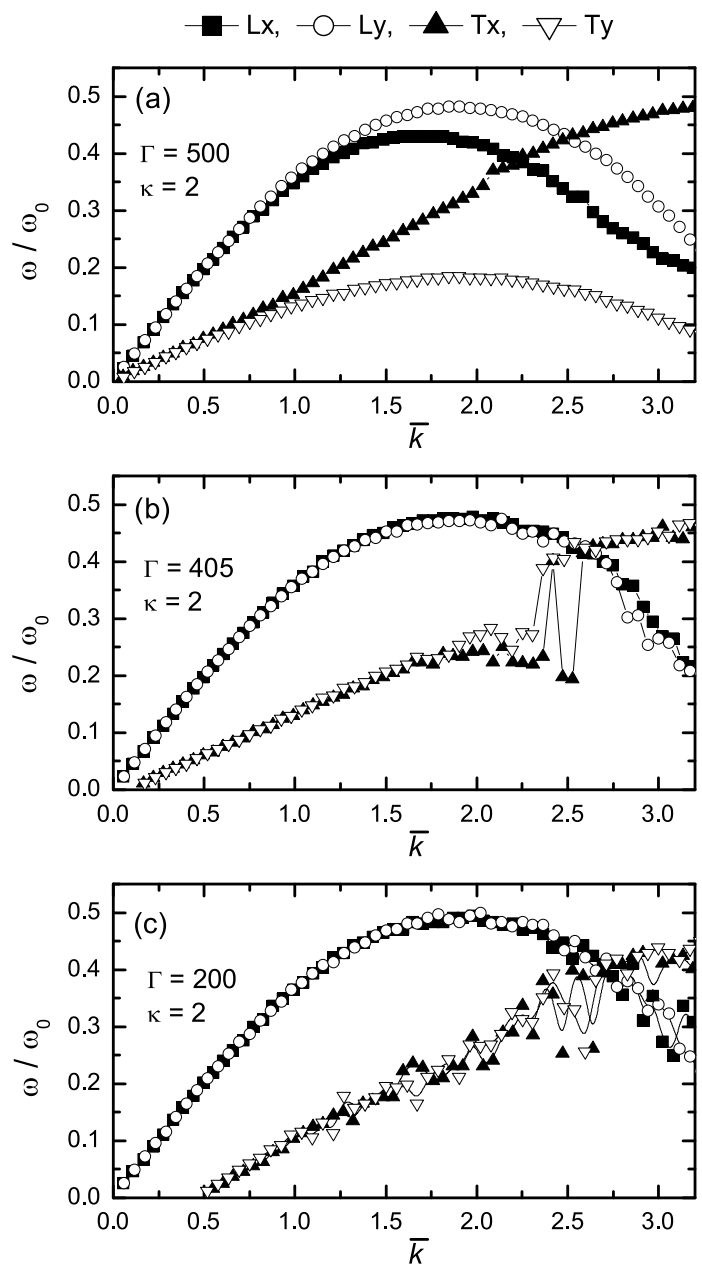

Figure 20. 2D Yukawa systems: Comparison of MD ( $\mathrm{L}$ and $\mathrm{T}$ ) dispersions in the solid phase $(\Gamma=500)$, just below the melting transition $(\Gamma=405)$ and in the liquid phase $(\Gamma=200)$ for $\kappa=2$. Shown are both $x$ and $y$ polarizations, where $x$ is in the direction to the nearest neighbor in the hexagonal lattice. Reproduced from Ref. [82. Copyright (2007) by the Institute of Electrical and Electronics Engineers.

system is a typical strongly coupled liquid. Most prominent features are the isotropy of the dispersion ( $x$ and $y$ directions are equivalent), and the appearance of a finite wavenumber cut-off for the $T$ mode.

The behavior of the velocity autocorrelation function $Z(t)$ of the $2 \mathrm{D}$ liquid is very similar to its $3 \mathrm{D}$ counterpart, at least for short times. Representative $Z(t)$ functions obtained at different screening parameter values are shown in figure 21(a). These functions, when plotted against $\omega_{\mathrm{E}} t$ exhibit nearly universal behavior [see figure 21(b)], indicating the relevance of the Einstein frequency in determining the single particle properties [32. While the in-depth analysis of the long-time behavior of the velocity autocorrelation function is beyond the scope of this paper, it is noted that in lowdimensional systems $Z(t)$ may exhibit a slow power law decay, which makes it nonintegrable [99]. As a consequence the diffusion coefficient may not exist for some 2D 

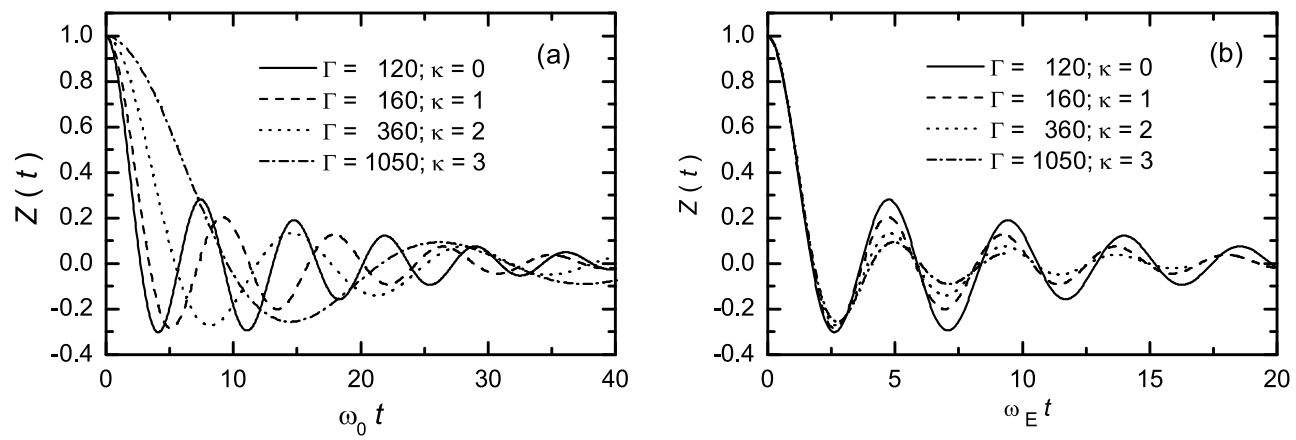

Figure 21. 2D Yukawa and Coulomb liquids: (a) velocity autocorrelation functions for a series of $\kappa$ values. (b) The same data as a function of $\omega_{\mathrm{E}} t$. The $(\Gamma, \kappa)$ pairs correspond to the same effective coupling $\Gamma^{\star}$. Reproduced from Ref. 32. Copyright (2005) by the American Physical Society.
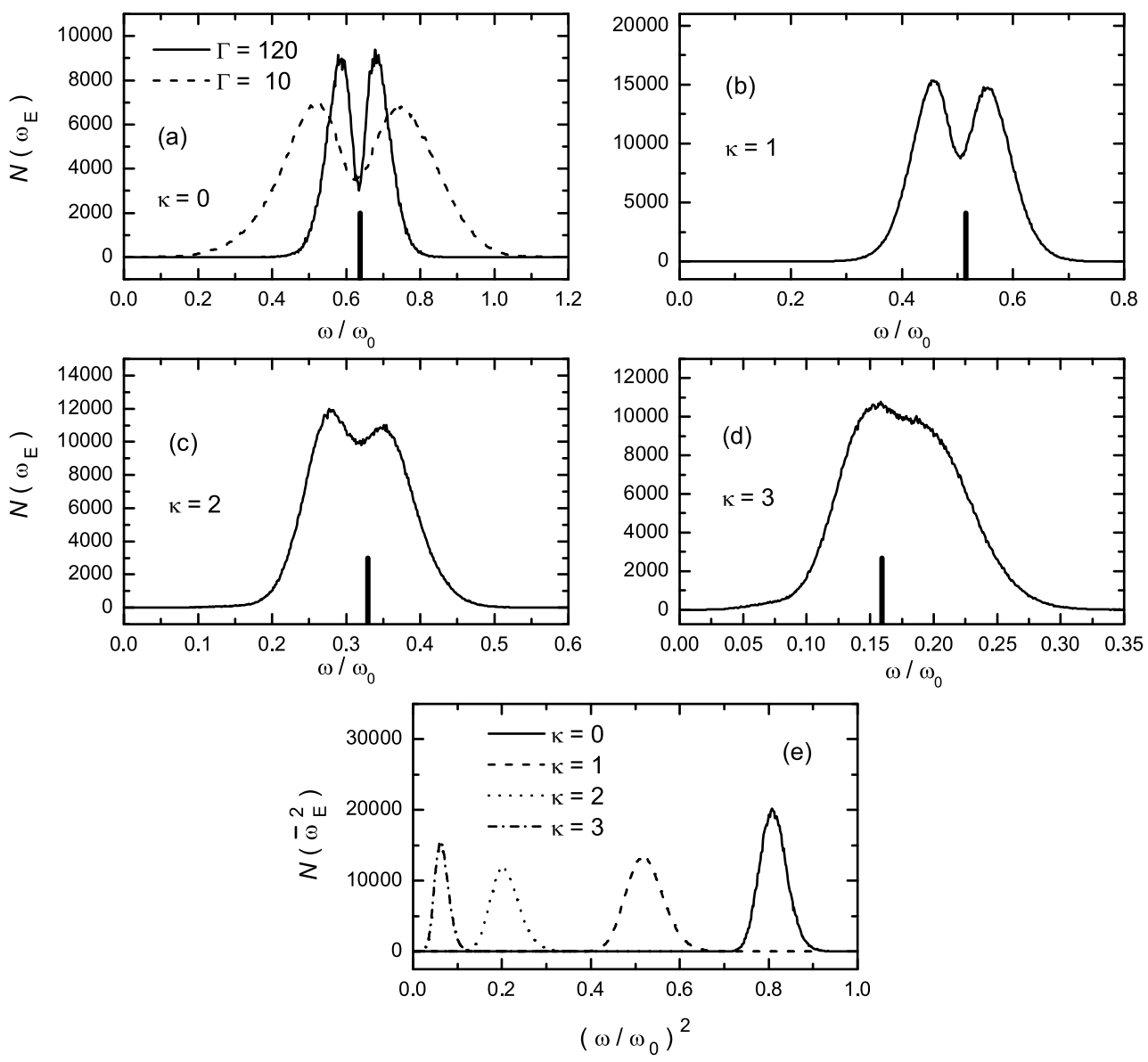

Figure 22. 2D Yukawa and Coulomb liquids: Einstein frequency distributions for $\Gamma^{\star}=120$ : (a) $\Gamma=120, \kappa=0$; (b) $\Gamma=160, \kappa=1$; (c) $\Gamma=360, \kappa=2$; (d) $\Gamma=1050$, $\kappa=3$; the vertical bars indicate values obtained by the QLCA theory. (a) also shows the frequency distribution obtained at a lower coupling value $\Gamma=10$. (e) Distribution of $\bar{\omega}_{\mathrm{E}}^{2}$ for the same systems. 


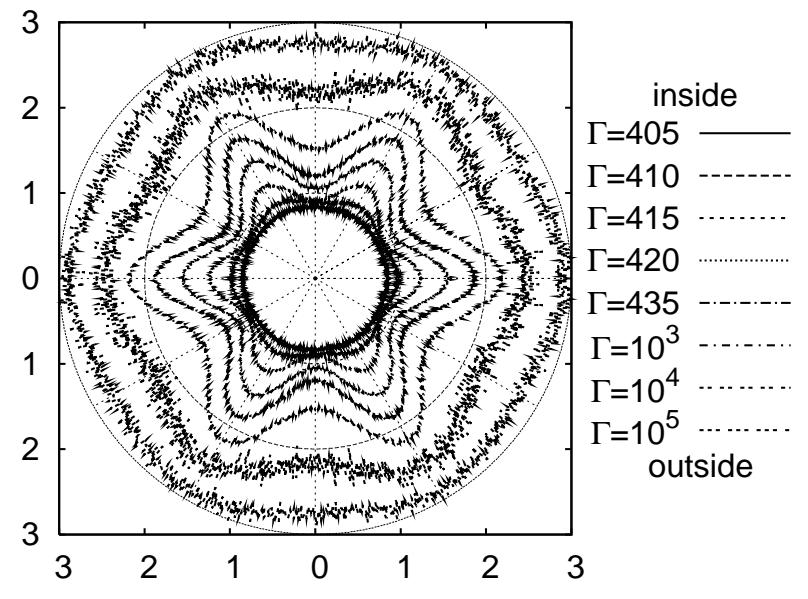

Figure 23. 2D Yukawa systems: distribution of the polarization angle for the higher frequency normal mode for different values of the coupling parameter $\Gamma$ across the crystallization boundary. $\kappa=2$. Reproduced from Ref. [104. Copyright (2007) by the Institute of Electrical and Electronics Engineers.

systems. The case of 2D Yukawa liquids has attracted considerable attention during the last years [100, 101, 102, 103]. These studies have found very nearly $Z(t) \propto t^{-1}$ decay of the velocity autocorrelation function and superdiffusion to exist for some conditions.

Similarly to the 3D case, numerical experiments were also performed for the 2D case to determine the distribution of the microscopic Einstein frequencies. A series of frequency histograms for $\Gamma^{\star}=120$ at different values of $\kappa$ are shown in figures 22(a)-(d). We observe two peaks in the distributions, which gradually get wider with increasing $\kappa$. The QLCA results are again in very good agreement with the mean values of the distributions. A wider frequency distribution appears when $\Gamma$ is lowered [see figure 22(e)]. The distributions of the sums of the 2 microscopic squared Einstein frequencies $\bar{\omega}_{\mathrm{E}}^{2}$ - as shown in figure 22(e) - are in contrast with the 3D situation. Here we do not find qualitative difference between the $\kappa=0$ Coulomb and the $\kappa \neq 0$ Yukawa cases for reasons discussed in section 3 .

The angular distribution of the polarization vector of the higher frequency normal mode oscillation has also been analyzed, as an indicator of the prevailing disorder [104]. Figure 23 shows the distribution of the polarization angle for the higher frequency normal mode for different values across the crystallization boundary (at $\Gamma \cong 415$ ). As discussed in section 3, both the liquid (away from the phase transition boundary) and the perfect lattice (extremely high $\Gamma$ values) exhibit a full rotational symmetry, while in between the sixfold symmetry of the lattice prevails.

The diagram showing the widths of the spectra of the dynamical structure functions is displayed in figure 24. Comparison with data for the 3D Yukawa liquid reveals that the trends and orders-of-magnitudes in the two cases are not substantially different. The dependence of the width of the peaks in the $S(k, \omega)$ spectra as a function of the reduced coupling parameter follows the form $\Delta \omega / \omega_{0} \cong 0.76\left(\Gamma^{\star}\right)^{-2 / 3}$, as it is shown in figure 25. 

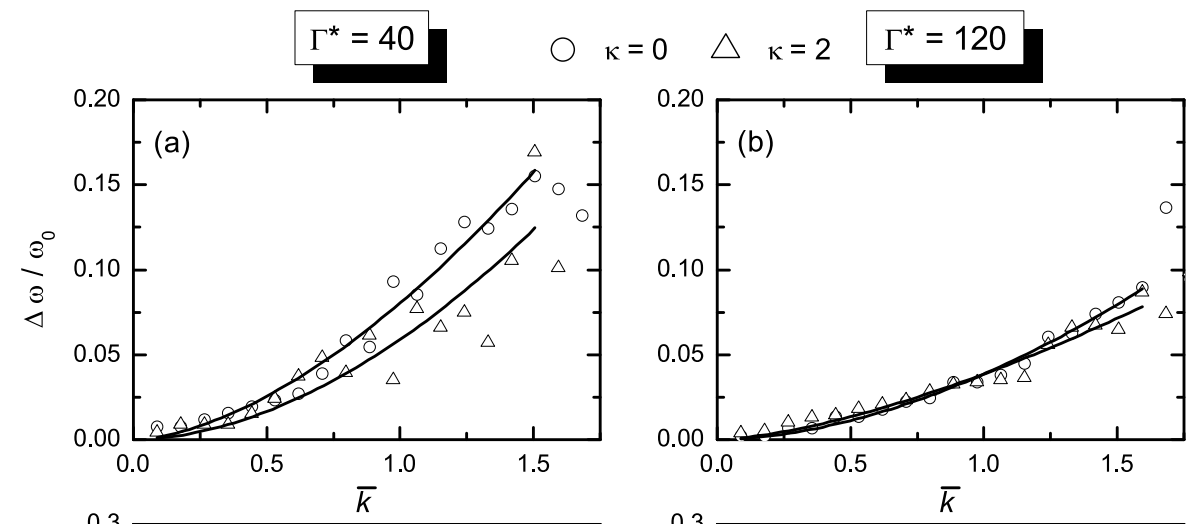

S
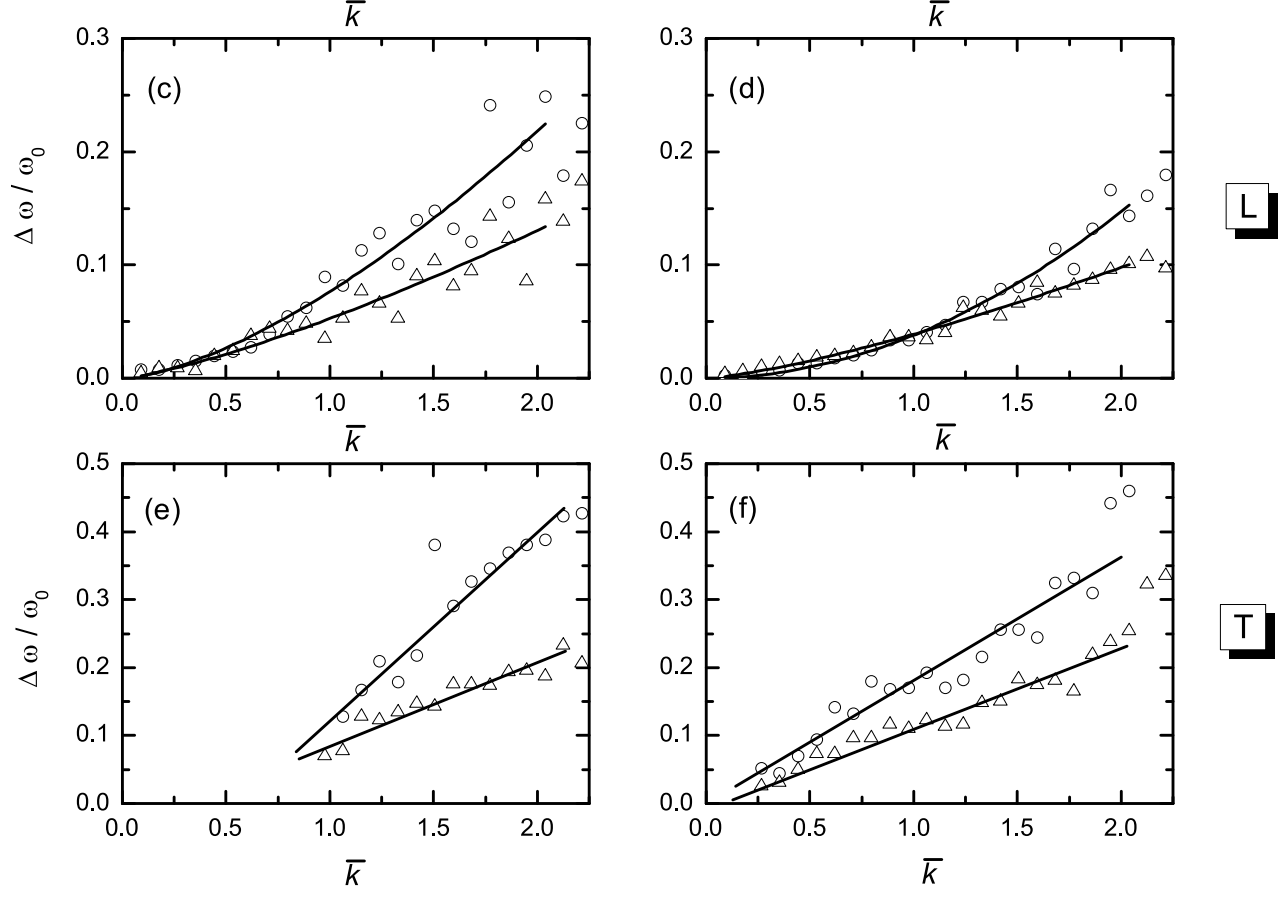

Figure 24. 2D Yukawa and Coulomb liquids: spectral line width for $\kappa=0$ and $\kappa=2$ at $\Gamma^{\star}=40(\mathrm{a}, \mathrm{c}, \mathrm{e})$ and $\Gamma^{\star}=120(\mathrm{~b}, \mathrm{~d}, \mathrm{f})$. Shown are FWHM (full-width at halfmaximum) values for the most prominent peaks in the $S(k, \omega), L(k, \omega)$ and $T(k, \omega)$ spectra.

This monotonically decreasing function of the coupling may change character at lower coupling values, for which data are at present not available. This is expected on the basis of the prediction by Hansen et al. [50] (for the 3D case): for weak coupling the width is expected to increase from its Vlasov value where $S(k, \omega)$ should be extremely sharp, since higher coupling leads to higher collision frequency and thus to stronger damping. Once, however, localization sets on, further increase in the coupling is expected to create better localization and thus a reduction in the collision frequency and in the width. Thus, generation of data for lower $\Gamma$ values would be desirable, to see whether a turnaround point really exists. 


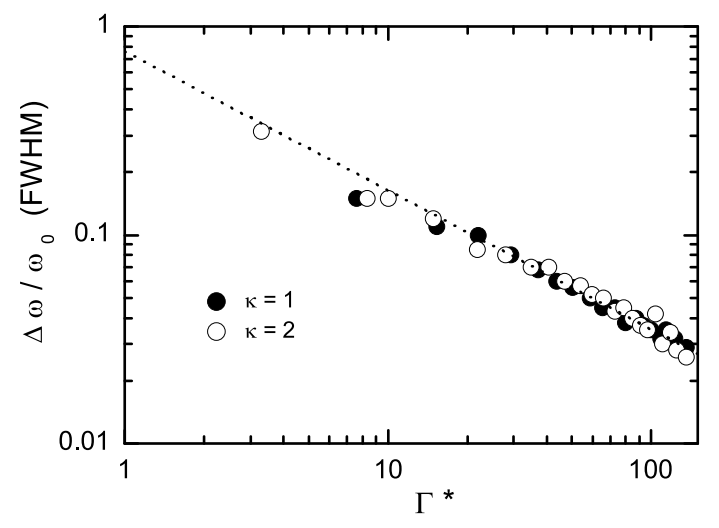

Figure 25. 2D Yukawa and Coulomb liquids: line widths of the $S(k, \omega)$ spectra as a function of the reduced coupling parameter $\Gamma^{\star}$, at a fixed wave number $\bar{k}=1.0$.

\subsection{Quasi-two-dimensional Yukawa liquids confined by a parabolic potential}

The model adopted for the 2D Yukawa system, which assumes that the particles are constrained to move entirely within an ideal plane can be extended to describe more accurately the situation found in physical systems, by allowing small amplitude displacements of the particles perpendicular to the plane. In this extended model one applies a parabolic potential along the direction perpendicular to the plane, which then results in a quasi-two-dimensional confinement. Such confinement gives rise to a particle layer with finite width, or - at weaker confinement - to a sequence of multilayer structures, when the confinement or interaction potential is varied. The structural phase transitions (a change in the number of layers and in the accompanying crystal structures), relevant to particle traps, have theoretically been studied by Dubin [105], while Totsuji et al. [106], Bystrenko [107] as well as Qiao and Hyde [108] investigated the formation of layers in Coulomb and Yukawa systems in confined quasi-2D configurations.

The number of layers formed in the liquid phase depends on the strength of the confinement. In contrast to the idealized 2D systems the layers have a finite width. Here we deal with the domain of parameters when a single layer is formed. At higher number of layers the mode structure is expected to be more complex [109, 110, 111, but the study of these modes is not within the scope of the present analysis. In a single-layer configuration the third degree of freedom of the particles, the displacement perpendicular to the plane, gives rise to an additional collective excitation, the "out-of-plane" $\mathcal{P}$ mode, besides the "in-plane" $\mathcal{L}$ and $\mathcal{T}$ modes found in (ideal) 2D layers. The out-of-plane mode in the crystallized state has been studied through simulations by Qiao and Hyde [112]. Results pertaining the strongly coupled liquid phase were analyzed in [113], and will be summarized below. It should also be noted that a somewhat similar physical situation arises when a 1D chain of particles is confined in the transverse direction by a parabolic potential: indeed, there is a similarity between the modes that represent excursions along the direction of the confining force in the 1D and 2D systems.

In the quasi-2D liquid system the particles can freely move in the $(x, y)$ plane while 
a confinement potential $V_{c}(z) \propto z^{2}$ acts upon them when they are displaced from the $z$ $=0$ plane. The confinement force is linear with respect to the "vertical" displacement,

$$
F_{z}=-f_{0} \frac{Q^{2}}{4 \pi \varepsilon_{0} a^{3}} z,
$$

where the strength $f_{0}$ (besides $\Gamma$ and $\kappa$ ) is the third characteristic parameter of the system. At $f_{0}=1$ the confinement force at a vertical displacement $z=a$ is equal to the magnitude of the force between two particles separated by $a$ [defined by (5)], interacting via Coulomb potential. Information about the (thermally excited) collective modes and their dispersion is obtained from the analysis of the correlation spectra of the longitudinal and (in-plane as well as out-of-plane) transverse current fluctuations. For the "in-plane" $\mathcal{L}$ and $\mathcal{T}$ modes we use eq. (11), while for the out-of-plane mode the corresponding microscopic current $\pi(k, t)$ (which characterizes the $\mathcal{P}$ mode) is obtained as:

$$
\pi(k, t)=k \sum_{j} v_{j z}(t) \exp \left[i k x_{j}(t)\right] .
$$

Representative current fluctuation spectra for the three $(\mathcal{L}, \mathcal{P}$, and $\mathcal{T})$ modes are displayed in figure 26, for $\Gamma=100, f_{0}=2.0$ and two different values of the screening parameter $\kappa=0.27$ and $\kappa=1.33$. The frequency is normalized according to (7). We observe sharp peaks in the $L(k, \omega)$ spectra, similarly to the case of (ideal) 2D Coulomb and Yukawa liquids [52, 71], characteristic of long-lifetime collective excitations [114]. Peaks in the $\mathcal{T}$ mode spectra [see figure 26(g,h)] show up only above a certain (cutoff) wave number, similarly to the case of 2D and 3D Yukawa systems, as discussed in the previous sections.

The $\mathcal{P}$ mode possesses a finite frequency at $k=0$, which is, in general, characteristic of an optical mode. The first identification of this pseudo-optical behavior in a confined $2 \mathrm{D}$ system is due to [114]. At small wave numbers the peaks of the spectra shift to lower $\omega$ as $\bar{k}$ is increased. The width of the peaks of the $P(k, \omega)$ spectra become gradually broader when $\kappa$ is increased, as it can be seen in figure 26(e) and (f). It is noted that, on the other hand, the peaks become narrower as the strength of the confining potential, $f_{0}$, is increased, which is an indication of an increasing lifetime of this collective excitation.

The dispersion relations derived from the spectra are displayed in figure 27 for different values of $f_{0}$ and $\kappa$ for $\Gamma=100$. At constant $\kappa$, as shown in figure 27(a), the frequency of the out-of-plane mode changes significantly as the strength of the confinement force, $f_{0}$, is varied. The $\mathcal{L}$ and $\mathcal{T}$ modes are only slightly affected by the value of $f_{0}$. The frequency of these modes is somewhat smaller at $f_{0}=1.4$, which is near the lower bound of $f_{0}$ for the formation of a single layer [113]. It is noted that at lower $f_{0}$ values, when two layers are formed, two longitudinal and two in-plane transverse modes appear, similarly to those identified in the classical (ideal) bilayer system [109]. Additionally, two out-of-plane transverse modes also emerge in the two-layered system, which are also believed to be in-phase and out-of-phase modes (when particles in the two layers oscillate in phase or with a phase difference of $180^{\circ}$ in the two layers). The 

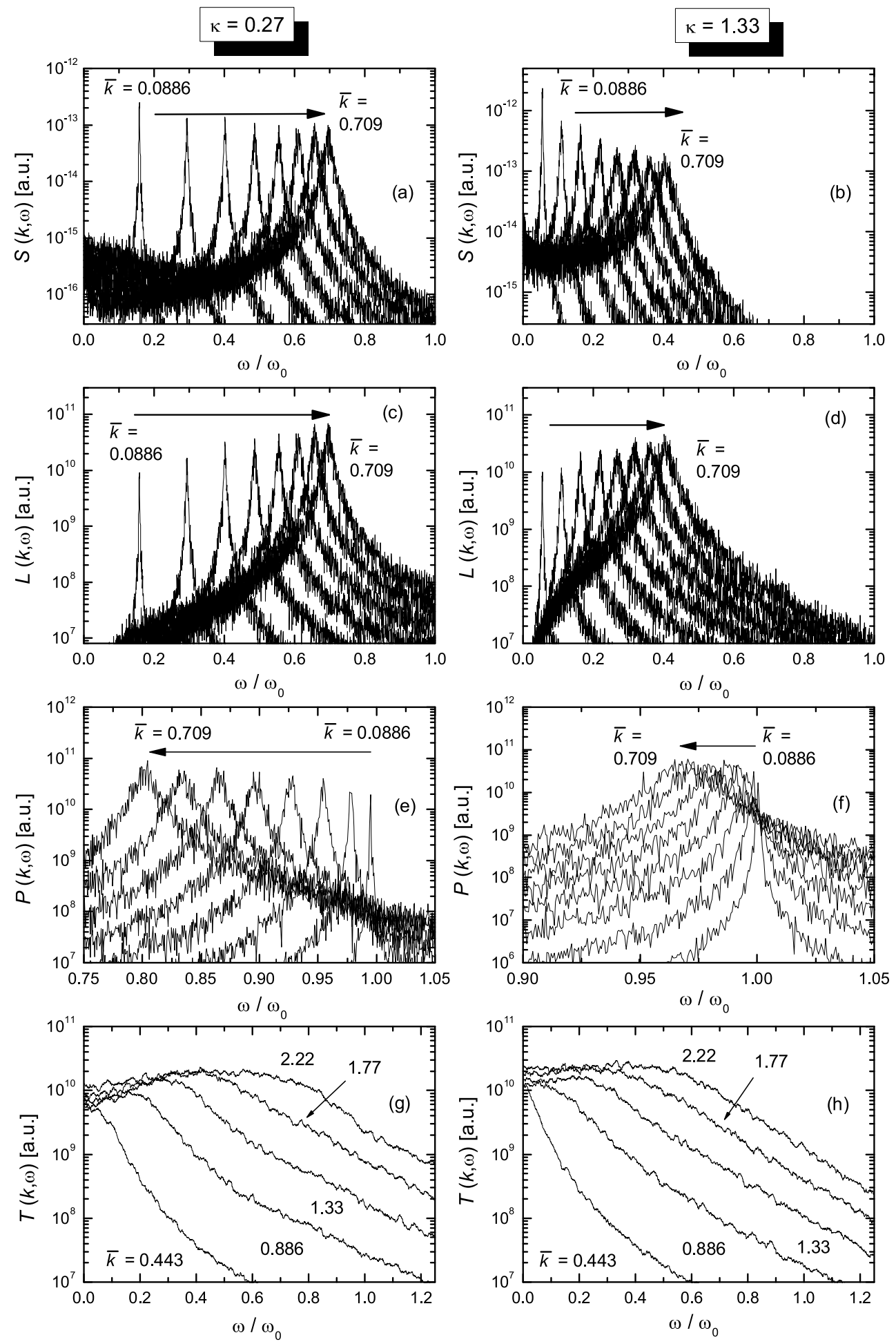

Figure 26. Quasi-2D Yukawa liquids: dynamical structure function $[S(k, \omega)]$, longitudinal $[L(k, \omega)]$ and out-of-plane as well as in-plane transverse $[P(k, \omega)$ and $T(k, \omega)]$ current fluctuation spectra for $\kappa=0.27$ (a,c,e,g) and $\kappa=1.33$ (b,d,f,h). The $S$, $L$, and $P$ spectra are plotted for the multiples of the smallest accessible wave number $\bar{k}_{\text {min }}=0.0886$, while the $T$ spectra are shown for higher wave numbers indicated by the labels in (g) and (h). The arrows in (a)-(f) indicate increasing wave numbers. Confining force: $f_{0}=2$. Reproduced from Ref. [113]. Copyright (2004) by the American Physical Society. 

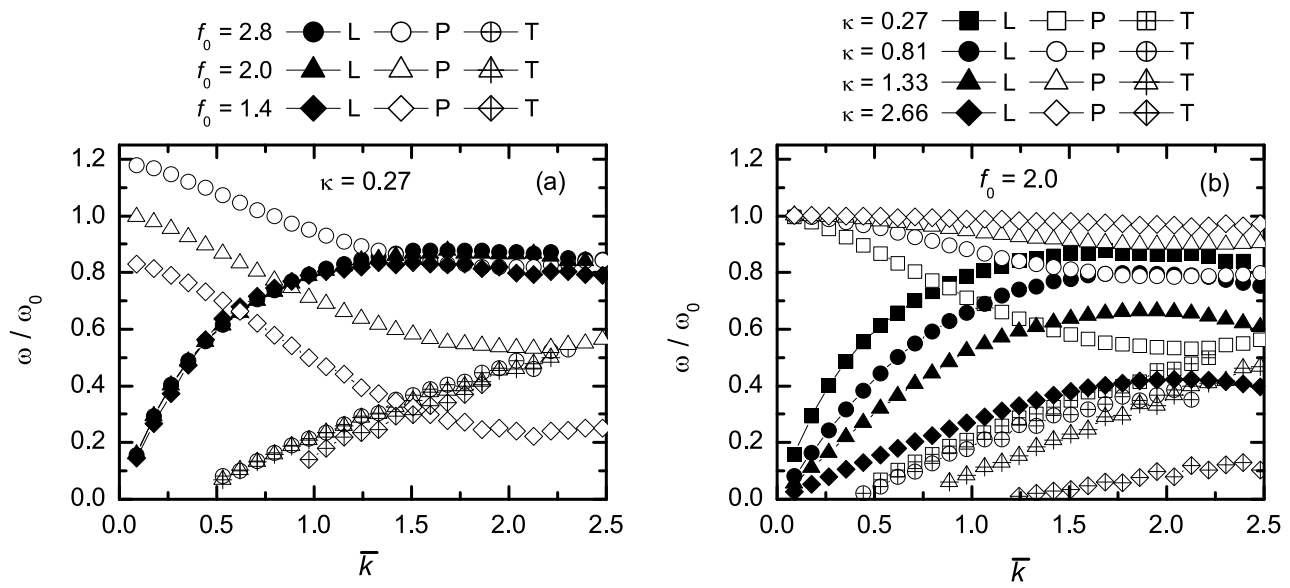

Figure 27. Quasi-2D Yukawa liquids: dispersion relations for (a) $\kappa=0.27$ and different values of the amplitude $f_{0}$ of the confining potential, and (b) for fixed $f_{0}$ $=2$ and different values of the screening parameter. Reproduced from Ref. [113]. Copyright (2004) by the American Physical Society.

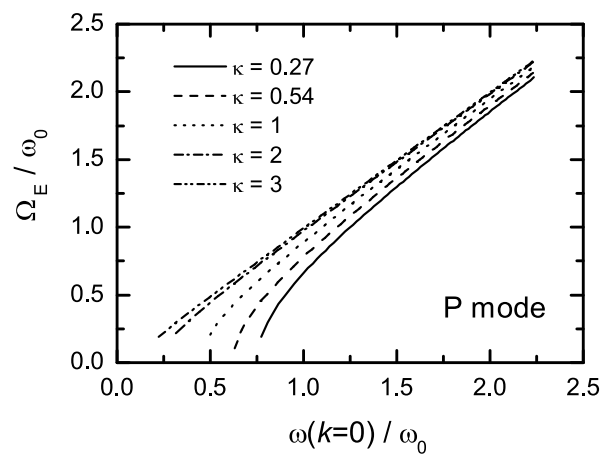

Figure 28. Quasi-2D Yukawa liquids: the relation between the frequency $\omega(k=0)$ and the Einstein frequency of the $\mathcal{P}$ mode for different values of the screening parameter $\kappa$. Reproduced from Ref. [113. Copyright (2004) by the American Physical Society.

$\mathcal{L}$ mode exhibits a quasi acoustic behavior, with a linear portion of the dispersion curve around $k=0$, which widens with increasing $\kappa$, as it can be seen in figure 27(b). The $\mathcal{T}$ mode shows an acoustic, $\omega \sim k$ dispersion at small $k$, with a cutoff at a finite wave number.

For the $\mathcal{P}$ mode $\mathrm{d} \omega / \mathrm{d} k<0$ in the $\bar{k} \lesssim 2.1$ domain. At higher wave numbers, the frequency of the mode slightly increases with $k$. This observation on the liquid system agrees well with that on the crystallized system [112], where the same behavior was found, except that in the latter system the critical wave number (at which the group velocity $\mathrm{d} \omega / \mathrm{d} k$ changes from negative to positive) also depends on the direction of the propagation.

At $k=0$ the whole layer oscillates in unison in the potential well with a frequency:

$$
\frac{\omega(k=0)}{\omega_{0}}=\sqrt{f_{0} / 2} .
$$


A smaller confinement force results in a smaller $\omega(k=0)$ and $\omega(k \rightarrow \infty)$. At a constant $f_{0}$ the value of $\omega(k=0)$ does not change when $\kappa$ is varied, but - as shown in figure 27(b) $-\omega(k>0)$ increases with decreasing $\kappa$. This is explained by the decreased interparticle force (at an average particle separation) at higher $\kappa$.

The frequency of the out-of-plane mode at the $k \rightarrow \infty$ limit (i.e. the Einstein frequency [35, 33]) can be calculated by considering the forces acting upon a single particle displaced in the $z$-direction, while all other particles are in rest in the $z=0$ plane. The force is the sum of the confining force and the force due to repulsion by the other particles,

$$
F(z)=-f_{0} \frac{Q^{2}}{4 \pi \varepsilon_{0} a^{3}} z+F_{\mathrm{r}}(z) .
$$

The $F_{\mathrm{r}}(z)$ contribution can be calculated as $F_{\mathrm{r}}(z)=-\partial V_{\mathrm{r}} / \partial z$, where $V_{\mathrm{r}}(z)$ is the potential distribution due to a charge distribution $\rho(x, y)$ in the $z=0$ plane. To obtain $\rho(x, y)$ one may either use the radial (2D) pair correlation function (PCF) or consider the particles occupying hexagonal lattice sites in the $z=0$ plane. The $F_{\mathrm{r}}(z)$ force is found to be a nearly linear function of the displacement $z$, in the $|z|<0.3 a$ domain, where the particle displacement is expected to fall. The resulting (Einstein) frequency (when the particles in the $z=0$ plane are situated at lattice sites) is [113]:

$$
\frac{\Omega_{\mathrm{E}}}{\omega_{0}}=\frac{\omega(k \rightarrow \infty)}{\omega_{0}} \cong \sqrt{\frac{f_{0}-1.63 \exp (-1.37 \kappa)}{2}} .
$$

In the case of using the disordered configuration in the $z=0$ plane instead of lattice sites (through PCFs obtained in the liquid state simulations), a frequency very close to that given by (62) is obtained. At low values of $\kappa$ the Einstein frequency $\omega_{\mathrm{E}}$ is significantly lower than $\omega(k=0)$, as illustrated in figure 28, In the high $\kappa$ limit the two frequencies are equal, as the screening becomes very strong and the particles interact very weakly. In this case the frequency of the $\mathcal{P}$ mode becomes nearly independent of $\bar{k}$.

\section{Experimental results}

Experimental results on wave propagation and collective excitations in Yukawa systems have been accumulating in complex (dusty) plasma experiments since the mid 1990-s. Experiments have been carried out both on spontaneously generated and on externally excited waves. An early laboratory observation of longitudinal modes was reported by Barkan et al. [115] in 1995, followed by a more detailed study in 1997 [116]: these authors observed spontaneously generated waves in dust that filled a volume with a cylindrical geometry. These waves grew as the result of the dust-acoustic instability, which was driven by an ion flow, and the experimenters were able to measure the wavelength and propagation speed. An early effort to excite waves by manipulation using an electrically-biased wire was reported by Pieper and Goree [117, who also introduced a method of data analysis that yields the real and imaginary parts of the wave number, for the applied frequency. Repeating the measurements at various frequencies yielded a 
dispersion relation. In some of these early experiments the ambient pressure was kept high in order to avoid instabilities. As a result, as pointed out by Rosenberg and Kalman [72], the waves were strongly damped, primarily by grain-neutral collisions. Thus, even though the experiments were conducted under strongly coupled conditions in the liquid state, the strong collisional damping washed away the difference between weakly coupled and strongly coupled dispersions (see [64, 72] for a more detailed discussion). Two recent experiments have further corroborated this picture. Bandyopadhyay et al. [118] investigated the acoustic dispersion of the longitudinal mode in the strong coupling regime over a wide range of the neutral pressure values and found $\partial \omega / \partial k<0$ behavior of the dispersion curve that in the low collisional domain could be attributed to correlational effects. On the other hand, the experiment reported by Annibaldi et al. [119], in the high collisional regime confirmed that in this domain the strong coupling effects were washed away completely.

The first experiments where strong coupling effects were clearly displayed were done on a 1D complex plasma in the crystalline state, realized as a chain of grains held together in the transverse direction by a confining potential. Longitudinal waves (along the direction of the chain) excited by the radiation pressure of a laser beam [120, 121] in a parallel plate radio frequency discharge were observed: the analysis led to the conclusion that the weakly coupled theory of the longitudinal waves (referred to as "dust acoustic waves" [122]) was inadequate, while the description in terms of harmonic phonons of a system with short range interaction (referred to as "dust lattice waves" [123]) provided a more satisfactory agreement with experiments.

Generation of 3D complex plasmas in the laboratory under strong coupling conditions and at sufficiently low pressure, so that strong coupling effects become manifest turned out to be difficult. A good summary of the state of affairs as of 2000 is given by [64]. This paper and a later work [124] also discuss the spontaneous excitation of shear wave-like structures in a strongly coupled liquid at a low pressure. The plasma originally was in a 3D configuration, but assumed a layer structure in the course of the experiment, with particle excursions in the direction perpendicular to the layers. Thus it seems difficult to judge whether the observed waves were indeed shear waves or some more intricate excitation in the coupled layer system.

The presence of the ion beam traversing the dust plasma generated in lowpressure gas discharges can create issues that can not be approached within the Yukawa model. Because of the anisotropy introduced by the ion beam, the Yukawa interaction will be modified in the vertical direction (along the beam), but probably not too much in the horizontal plane [125, 126]. This scenario was supported by experiment [127, 128, 129, 130]. A further major problem due to the ion beam in the 3D geometry was identified by Joyce et al. [131, that in the low pressure domain, where collective modes could be observable, ion-dust instability may lead to melting. To avoid these problems, most of the subsequent laboratory experiments were to favor 2D geometries over three-dimensional ones: in a 2D system these problems should be absent. Since 1998 substantial progress in the understanding of the excitation and propagation of 
waves in 2D Yukawa systems has ensued. In the strong coupling regime the constituent grains are, in principle, either in the crystalline solid or in the liquid state. In fact, in addition to the formation of large scale ordered lattice structures a more common configuration is an aggregate of micro-crystals whose prevailing disorder is expected to make the behavior of the aggregate quite similar to that of the liquid state.

Longitudinal waves in a 2D dust plasma crystal were first observed experimentally in a parallel plate radio frequency discharge by Homann et al. [132]. The observation of transverse (in-plane) shear waves, the hallmark of strong coupling, excited by a chopped laser beam was reported by Nunomura et al. [85]. Their measurements of the dispersion relation revealed an acoustic, i.e., non-dispersive, character over the entire range of wave numbers measured, $(0.3<\bar{k}<1.2)$, at $\kappa \approx 0.74$, with transverse sound speed and Einstein frequency values in agreement with theory [79, 80].

A series of beautiful experiments on the generation of Mach cones in the wake of an object moving through a 2D dusty plasma crystal was also crucial albeit in an indirect way, in determining the strong coupling characteristics of these systems. It was unambiguously shown [133, 134, 135] that Mach cones appear when the velocity of the moving object (particle or laser spot) exceeds the longitudinal sound speed $s_{\mathrm{L}}$ in the medium. Subsequent observations [136] with object velocities below this limit, but above the transverse shear sound speed $s_{\mathrm{T}}$ (the ratio of the two speeds had the value $s_{\mathrm{L}} / s_{\mathrm{T}}=4.48$ in the experiment) also demonstrated the excitation of a small angle Mach cone sustained by the transverse mode.

More recent experiments done both on the 2D solid and liquid phases have been able to determine plasma parameters with sufficient accuracy and to perform measurements of great number of observables, so that detailed quantitative comparisons with the theoretical predictions have become possible. Experiments by Nunomura et al. 137. introduced laser manipulation, which avoided technical problems caused earlier by the electrical wires, and the dispersion relations were measured with greater accuracy. Subsequently Nunomura et al. [138] detected the spectra of self-generated longitudinal and transverse excitations along the two principal axes of a triangular lattice. The energy was concentrated along a well defined $\omega(k)$ curve, representing the measured dispersion relation. The data covered the $0<\bar{k}<3.3$ domain with $\kappa \approx 0.74$; our comparison with the theoretical dispersion curves calculated by Peeters and Wu [80] and by Sullivan et al. [83] shows excellent agreement with these data. A partial frequency spectrum (i.e. density of states), based on the kinetic energy contents of the 4 selected modes was also generated: while comparison with the calculated spectrum (see section 3) is possible, agreement beyond what is visible in figure 29 is not expected, since the theoretical spectrum includes all propagating modes [139]. In a subsequent work [140] the spectrum of waves was measured also for a number of directions in between the principal axes and over a much broader domain of wave number values: $0<\bar{k}<6.6$. In addition to the frequencies, the polarization angles of the modes were also determined (the mode polarizations can be described as "longitudinal" and "transverse" for propagation along the principal directions only). All these data show excellent agreement with theory 
[80, 83], see figure 30.

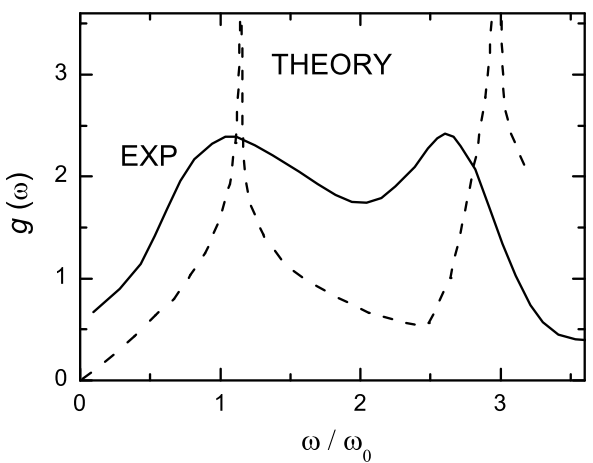

Figure 29. 2D system: density of states as obtained from the experiment of Nunomura et al. 138 and by theory.
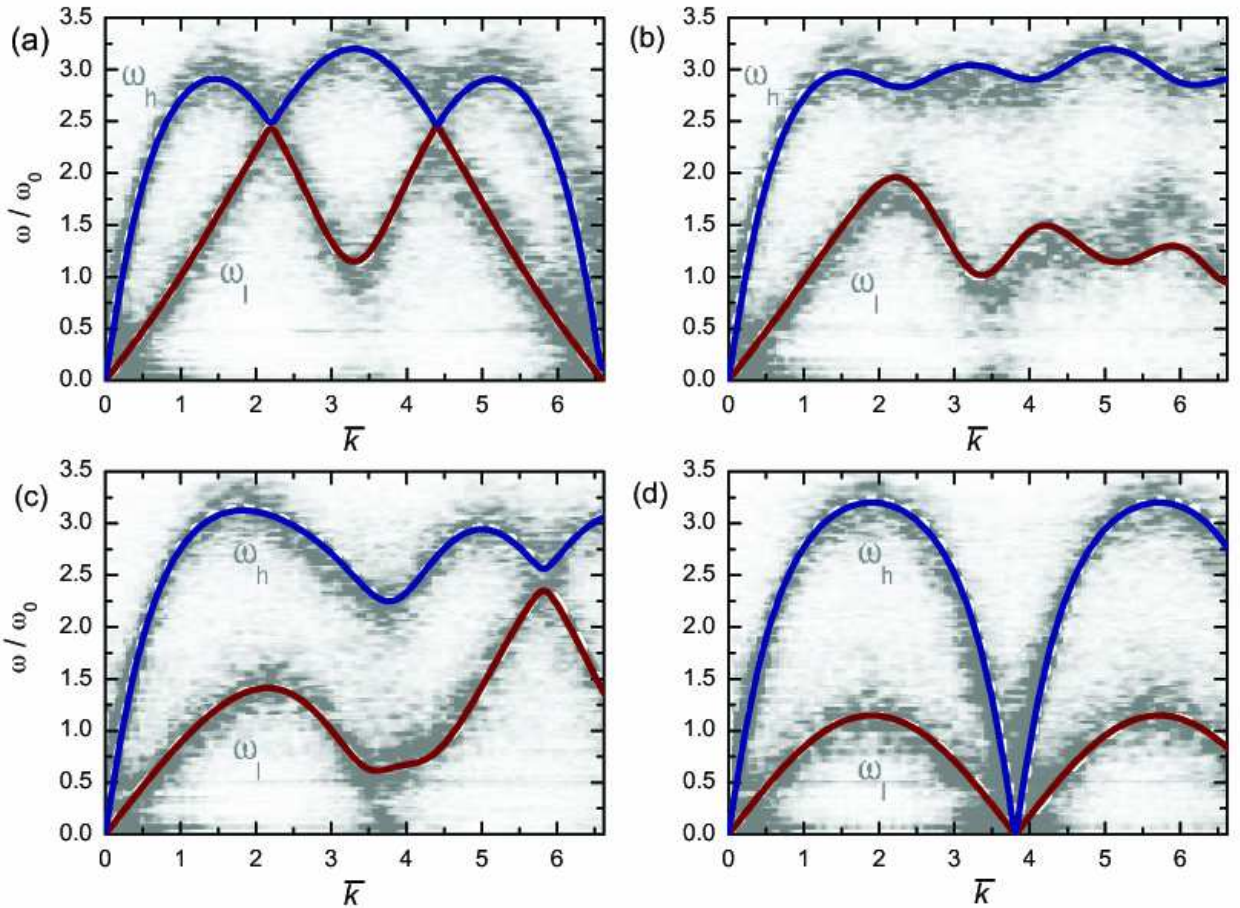

Figure 30. (color online) 2D system: wave dispersion in directions (a) 0, (b) 10, (c) 20, and (d) 30 degrees (measured from the nearest neighbor direction) as obtained in the experiment of Zhdanov et al. [140] and calculated from lattice summation (heavy lines). Experimental data reproduced from Ref. [140] with kind permission of the authors. Copyright (2003) by the American Physical Society.

Following a different line of approach Melzer [141] studied the normal modes of small 2D clusters of grains; what is of interest in the present context is the transition from the mode spectrum of a finite number of particles into that of an "infinite" 
system. With a somewhat arbitrary assignment of labels for the normal modes, it was found that the average frequency as a function of $k$ provides a fair resemblance to the $\omega(k)$ dispersion of the infinite lattice, already for a cluster as small as consisting of 34 particles. The scatter of frequencies around the $\omega(k)$ curve is of course substantially higher than in the experiments quoted above. (For somewhat related results see 34]). The theoretical understanding of the distribution of dynamical frequencies (as a function of temperature and particle number) presents a major theoretical challenge, with a very limited body of antecedents available in the literature [66, 142]. The possibility of generating experimentally observable scenarios from which information on the frequency distribution can be extracted should provide stimulus for new theoretical efforts.

As to the liquid state, observational data available at the present time are quite recent and still rather limited. Nunomura et al. [15] studied the change of the thermally excited mode structure as the crystal lattice was melted and the system transited to the strongly coupled liquid state. The melting was achieved by directed laser heating. The theoretically predicted trends, such as the development of a cut-off wave number for the shear mode and the shift of the longitudinal mode frequency towards higher values are in fair agreement with MD data. On the other hand, the widths of the spectra seem to be higher than expected. It is difficult to relate the results of the MD studies of the break-up and isotropization of the lattice modes [82 to this experiment, since the $\Gamma$ values where the observations of the liquid state were done are quite far from the phase transition point.

A careful study of the transverse modes in the strongly coupled liquid state, in the vicinity of the melting point is due to Piel et al. [16]. These authors analyzed the propagation of externally excited shear waves, through a sophisticated data analysis technique that made it possible to collect information from a high noise environment. The experimental situation corresponded to $\kappa \approx 0.4$, which allowed the comparison with the QLCA data for $\kappa=0$ and $\kappa \approx 1$ as lower and upper bounds. The authors found that within the wave number domain investigated $(0<\bar{k}<2.5)$ the overall agreement between experiment and the QLCA model is quite satisfying. They note that even in the solid state the waves assume characteristics resembling those in the liquid state (angularly averaged dispersion), because the plasma crystal consists of domains of different orientations. For this reason there does not seem to be too much change in the dispersion, as one passes from the solid phase to the liquid phase. On the other hand, the damping is substantially higher on the liquid side and becomes stronger for low $k$ values.

Since the homogeneous liquid cannot sustain shear, the shear mode must vanish below some finite $k_{\mathrm{c}}$ value. The value of this cut-off wave number was recently studied by Nosenko et al. [17] in a low pressure experiment. At the $\kappa \approx 0.43$ of the experiment $\bar{k}_{\mathrm{c}}$ ranges between 0.16 and 0.31 , as the coupling strength $\Gamma$ is varied from the melting value $\Gamma=155$ down to $\Gamma=60$. These values compare favorably with the values obtained by the MD simulations reported in [71] (see fig. [31). This can be taken as an indication that the cut-off is attributable to the intrinsic dynamics of the grains. Thus one can 
conclude that at low pressures the contribution of the grain-neutral collisions to the generation of the cut-off is quite negligible.

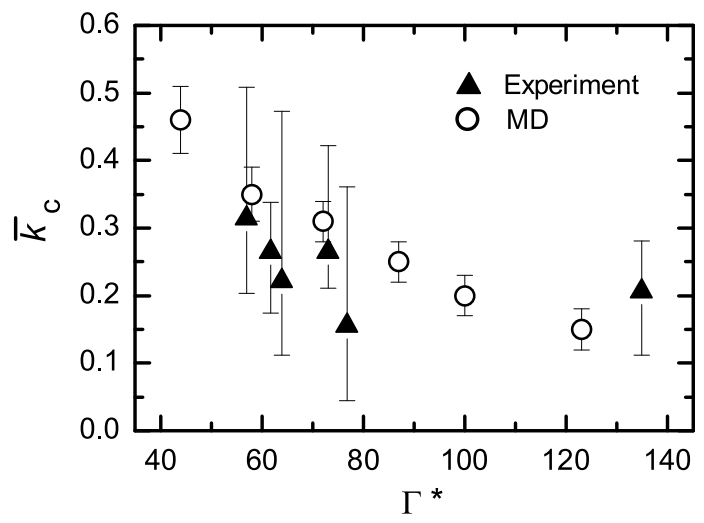

Figure 31. 2D Yukawa liquids: Transverse mode cutoff wavenumber $\bar{k}_{\mathrm{c}}$. Experimental values are taken from [17 and are compared with 2D Yukawa molecular dynamics results.

There are experiments on 1D chains of grains held together in the transverse direction by a confining potential which reveal (in addition to the observation of longitudinal waves on such systems quoted above [120, 121]) excitations in the direction perpendicular to the axis of the chain. These modes bear physical features similar to the shear-waves in 2D systems. In particular, they exhibit the benchmark pseudooptic behavior and the ensuing negative dispersion predicted by theory for the latter [108, 112, 113. Experimentally, the mode dispersion was determined by analyzing spontaneously excited waves by Misawa et al. [143] and by creating the transverse waves through the manipulation of a single particle by Liu et al. [144, 145] confirming these predicted features. In the 2D geometry, self-excited out-of-plane oscillations of particles were identified first by Nunomura [146]. Samsonov et al. [147] investigated the propagation of wave packets in the vertical (i.e. along the confinement) direction (see Section 4.3 for more details) and confirmed the predicted dispersion characteristics $[108,112$, 113] of the out-of-plane $(\mathcal{P}$, pseudo-optic) mode.

\section{Summary}

The objective of this review has been to summarize the huge body of information that has been gathered since 1990-s through theoretical analysis, computer simulations and laboratory experiments on the collective excitations of dusty (complex) plasmas and from this to determine the collective behavior of two- and three-dimensional strongly coupled Yukawa systems. The Yukawa model allows the mathematical analysis of an idealized system that represents a variety of actual many-particle physical systems (dusty plasmas, charged colloids, mesoscopic particles, etc.), which are characterized by (i) a significant ratio of the potential energy (originating from the high charge value 
of the particles) to the kinetic energy in the system, as expressed through the plasma coupling parameter $\Gamma$, and (ii) by a particle-particle interaction that is strongly affected by a polarizable background coexisting with the main plasma.

Two techniques have been used for the mathematical modeling: molecular dynamics, as a computational simulation method and the Quasi-Localized Charge Approximation as a theoretical scheme. The results generated by the two independent approaches have been found to be in excellent agreement with each other, and have been convincingly supported by the findings of laboratory experiments. Thus all these assembled data converge into a coherent and fairly complete physical picture, which has been presented in this Review. Nevertheless, there are quite a few areas that the reader may have expected to see in this paper, but which have been excluded from consideration. Thus some qualifying comments along this line are in order.

- We have considered infinitely large, unbounded 2- and 3-dimensional systems only: thus effects relating to 1D geometry, boundary conditions, inhomogeneities have been excluded. Some of these (Yukawa balls and disks e.g. [148, 149]) have been attracting much attention lately.

- A special configuration, familiar in semiconductor physics, is the bilayer geometry (consisting of two parallel 2D planes, separated from each other by a small distance d). While semiconductor devices are governed by Coulomb interaction, a similar configuration is of interest with systems where Yukawa interaction prevails [150]. The likelihood of the realization of such a geometry in laboratory dusty plasma experiments is not promising using identical grains, but may be more feasible in a microgravity environment. However, combining two species of differently sized microparticles in a conventional laboratory sheath geometry setup leads to the automatic formation of a bilayer configuration, due to the different $Z / m$ ratios, as recently pointed out by Matthews et al. [151] and demonstrated in a subsequent experiment by Smith et al. [152]. Bilayer systems posses rich variety of structural phases [150, 153, 154] and a rather complex collective mode structure whose details exhibit a remarkable sensitivity to the layer separation [109, 151, 155]. Further experimental investigation of this behavior would be of great interest.

- Only single component systems (the Yukawa equivalent of the OCP, one component plasma) have been treated; the crucially important extension to two- or multicomponent cases (different masses, different charges) is not here. Creation of such systems in the current laboratory set-ups is hampered by technical reasons (but again may become feasible in a microgravity environment) and serious theoretical studies are lacking.

- While we have presented simulation data of the dynamical fluctuation spectra in great detail, the evaluation and theoretical analysis of most of these data is still to be carried out. We have focused on the positions of the peaks in the spectra: the most important question amongst those whose analysis is incomplete is that of the widths of the peaks, which, in turn, are characteristic of the damping of the 
excitations. What is missing primarily is a solid theoretical foundation through which the different mechanisms that lead to the damping of the collective modes could be reconciled and against which the simulation data could be tested. The QLCA analysis points at the main physical effects where the source of the damping should be sought, but no reliable analytic tool has emerged that would predict how the damping depends on the coupling strength and on the wavelength of the mode.

- The theoretical tool (the QLCA) described in this Review is geared to strong coupling and it provides no linkage from the localization dominated strongly correlated behavior to the fluid-like weakly correlated behavior of the collective modes. Only more simulation work and a different theoretical approach would bridge this gap.

- There are both some experimental [140] and simulation (see figure 8] and [32]) results available on the effect of the disorder on the collective mode spectrum. Both these and theoretical considerations suggest that one should think in terms of frequency distributions, rather than in terms of well-defined collective mode dispersions.

- We have not discussed effects and phenomena relating to external or internally generated magnetic fields. These issues may become the topics of investigation for the next generation of complex plasma experiments. An externally imposed magnetic field could affect the polarizable medium (electrons and ions) and thus alter the effective interaction potential; at sufficient strength it may even change the orbits of the mesoscopic plasma particles and thus the prevailing mode structure [156, 157]. (As an example, consider a plasma with grains of $R=1$ micron, mass density $\rho \sim 1.5 \mathrm{~g} / \mathrm{cm}^{3}$ and $Z \sim 3000$; here a magnetic field of 2 Teslas would produce a dust cyclotron frequency $\omega_{\text {cd }} \approx 0.16 \mathrm{rad} / \mathrm{s}$, and with $T_{\mathrm{d}} \sim 0.03$ $\mathrm{eV}$ a gyroradius $r_{\text {gyro }} \approx 0.5 \mathrm{~cm}$. Thus, based on the criterion $r_{\text {gyro }}<$ confinement length, the creation of a magnetized plasma may become feasible. A more restrictive criterion may, however, emerge from the requirement $\omega_{\mathrm{cd}}>\nu_{\text {coll }}$, the grain-neutral collision frequency. In a different vein, systems containing magnetically polarizable plasma particles are expected to exhibit a series of novel physical phenomena, both in equilibrium [158] and in terms of collective excitations [159].

- Transport coefficients may have been a legitimate topic for consideration in this Review, but partly for reason of economy, partly because their treatment requires a different (theoretical and simulation) methodology from those appropriate for the study of wave phenomena, the subject has not been included. The transport coefficients of 3D Yukawa systems in the liquid phase are relative well known. The self-diffusion was studied in [94, estimates for the viscosity were given in [160]. Molecular dynamics simulations have proven to be invaluable tools for studies of transport phenomena and made possible the determination of shear viscosity and thermal conductivity [87, 161, 162, 163, 164, 165]. Recent theoretical work on this topic has focused on the mapping between Yukawa, Coulomb and hard-sphere systems [166, 167]. The effect of Langevin dynamics on the viscosity of 3D Yukawa 
systems has been studied in [168]. For recent experimental work on 3D systems see e.g. [169].

The realization of $2 \mathrm{D}$ complex plasma liquids and the development of modern experimental (perturbation and data acquisition) techniques resulted in renewed interest of transport properties (which are especially interesting due to the controversies about the very existence of transport coefficients in low-dimensional systems). During the last few years several experimental and simulation studies have been carried out on the shear viscosity [13, 14, 170, 171, 172, thermal conductivity [173, 174] and diffusion [100, 101] properties of 2D Yukawa liquids, and this topic is expected to attract further attention.

\section{Acknowledgments}

This work has been partially supported by OTKA-T-48389, MTA-NSF/102, OTKA-PD049991, OTKA-IN-69892, NSF PHY-0206695, NSF PHY-0715227, NSF PHY-0514619 and DE-FG02-03ER54716 grants. We thank Marlene Rosenberg, Stamatios Kyrkos, John Goree for numerous helpful contributions without which this Review would have been less complete, to Kenneth I. Golden for critically reading part of the manuscript and to Pradip Bakshi for discussions.

\section{References}

[1] Kalman G J, Rommel J M and Blagoev K 1998 Strongly coupled Coulomb systems (New York: Plenum Press)

[2] Löwen H, Allahyarov E, Likos C N, Blaak R, Dzubiella J, Jusufi A, Hoffmann N and Harreis H M 2003 J. Phys. A 365827

[3] Löwen H, Hansen J P and Roux J N 1991 Phys. Rev. A 441169

[4] Hynninen A P and Dijkstra M 2003 J. Phys. Condens. Matter 15 S3557

[5] Auer S and Frenkel D 2002 J. Phys. Condens. Matter 147667

[6] Konopka U, Morfill G E and Ratke L 2000 Phys. Rev. Lett. 84891

[7] Fortov V E, Ivlev A V, Khrapak S A, Khrapak A G and Morfill G E 2005 Physics Reports 4211

[8] Ishihara O 2007 J. Phys. D: Appl. Phys. 40 R121

[9] Thomas H, Morfill G E, Demmel V, Goree J, Feuerbacher B and Möhlmann D 1994 Phys. Rev. Lett. 73652

[10] Chu J H and I L 1994 Phys. Rev. Lett. 724009

[11] Quinn R A, Cui C, Goree J, Pieper J B, Thomas H and Morfill G E 1996 Phys. Rev. E 53 R2049

[12] Thoma M H, Kretschmer M, Rothermel H, Thomas H M and Morfill G E 2005 Am. J. Phys. 73 420

[13] Nosenko V and Goree J 2004 Phys. Rev. Lett. 93155004

[14] Liu B and Goree J 2005 Phys. Rev. Lett. 94185002

[15] Nunomura S, Zhdanov S, Samsonov D and Morfill G E 2005 Phys. Rev. Lett. 94045001

[16] Piel A, Nosenko V and Goree J 2006 Physics of Plasmas 13042104

[17] Nosenko V, Goree J and Piel A 2006 Phys. Rev. Lett. 97115001

[18] Chan C L, Lai Y J, Woon W Y, Chu H Y and I L 2005 Plasma Physics and Controlled Fusion 47 A273-A281

[19] Hamaguchi S 1999 Plasmas and Ions 257

[20] Slattery W L, Doolenn G D and De Witt H E 1980 Phys. Rev. A 212087 
[21] Slattery W L, Doolenn G D and De Witt H E 1982 Phys. Rev. A 262255

[22] Farouki R T and Hamaguchi S 1993 Phys. Rev. E 474330

[23] Brush S G, Sahlin H L and Teller E L 1966 J. Chem. Phys. 452102

[24] Stringfellow G S, DeWitt H E and Slattery W L 1990 Phys. Rev. A 411105

[25] Muto S and Aoki H 1999 Phys. Rev. B 5914911

[26] Gann R C, Chakravarty S and Chester G V 1979 Phys. Rev. B 20326

[27] Grimes C C and Adams G 1976 Phys. Rev. Lett. 36145

[28] Hamaguchi S, Farouki R T and Dubin D H E 1997 Phys. Rev. E 564671

[29] Vaulina O, Khrapak S and Morfill G 2002 Phys. Rev. E 66016404

[30] Vaulina O and Vladimirov S V 2002 Phys. Plasmas 9835

[31] Fortov V E, Vaulina O S, Petrov O F, Molotkov V I, Lipaev A M, Torchinsky V M, Thomas H M, Morfill G E, Khrapak S A, Semenov Y P, Ivanov A I, Krikalev S K, Kalery A Y, Zaletin S V and Gidzenko Y P 2003 Phys. Rev. Lett. 90245005

[32] Hartmann P, Kalman G J, Donkó Z and Kutasi K 2005 Phys. Rev. E 72026409

[33] Bakshi P, Donkó Z and Kalman G J 2003 Contrib. Plasma Phys. 43261

[34] Donkó Z, Kalman G J and Golden K I 2002 Phys. Rev. Lett. 88225001

[35] Donkó Z, Hartmann P and Kalman G J 2003 Phys. Plasmas 101563

[36] Golden K I and Kalman G J 2000 Phys. Plasmas 714

[37] Kalman G J, Golden K I, Donkó Z and Hartmann P 2005 J. Phys.: Conf. Ser. 11254

[38] Frenkel D and Smit B 1996 Understanding molecular simulation (San Diego: Academic Press)

[39] Baimbetov F B, Ramazanov T S, Dzhumagulova K N, Kadyrsizov E R, Petrov O F and Gavrikov A V 2006 J. Phys. A: Math. Gen. 394521

[40] Löwen H 1992 J. Phys.: Condens. Matter 410105

[41] Ewald P P 1921 Ann. Phys. 64253

[42] Sagui C and Darden T A 1999 Ann. Rev. Biophys. Biomol. Struct. 28155

[43] David N and Hooker S M 2003 Phys. Rev. E 68056401

[44] Gargallo R, Hünenberger P H, Avilés F X and Oliva B 2003 Protein Science 122161

[45] David N, Spence D J and Hooker S M 2004 Phys. Rev. E 70056411

[46] Eastwood J W, Hockney R W and Lawrence D N 1980 Comput. Phys. Commun. 19215

[47] Hockney R and Eastwood J 1981 Computer Simulation Using Particles (McGraw-Hill)

[48] Nosé S 1984 J. Chem. Phys 81511

[49] Hoover W G 1985 Phys. Rev. A 311695

[50] Hansen J P, McDonald I R and Pollock E L 1975 Phys. Rev. A 111025

[51] Barrat J L, Hansen J P and Totsuji H 1988 J. Phys. C: Solid State Phys. 214511

[52] Murillo M S and Gericke D O 2003 J. Phys. A: Math. Gen. 366273

[53] Murillo M S 2000 Phys. Rev. Lett. 852514

[54] Golden K I, Kalman G J and Silevitch M B 1992 J. Stat. Phys. 687

[55] Baus M and Hansen J P 1980 Physics Reports 591

[56] March N H 1984 Coulomb Liquids (London: Academic Press)

[57] Pines D and Nozieres P 1966 Theory of Quantum Liquids vol 1 (New York: W. A. Benjamin, Inc.)

[58] Murillo M S 1998 Phys. Plasmas 53116

[59] Murillo M S 2000 Phys. Plasmas 733

[60] Baus M 1977 Phys. Rev. A 15790

[61] Wallenborn J and Baus M 1978 Phys. Rev. A 181737

[62] Kaw P K and Sen A 1998 Physics of Plasmas 53552

[63] Ichimaru S, Iyetomi H and Tanaka S 1987 Physics Reports 14991

[64] Kaw P K 2001 Physics of Plasmas 81870

[65] Kalman G J and Golden K I 1990 Phys. Rev. A 415516

[66] Elliot R J, Krumhansl J A and Leath P L 1974 Rev. Mod. Phys. 46465

[67] Lado F 1978 Phys. Rev. B 172827 
[68] Golden K I, Kalman G J and Wyns P 1992 Phys. Rev. A 463463

[69] Golden K I, Kalman G J and Wyns P 1992 Phys. Rev. A 463454

[70] Kalman G J, Rosenberg M and DeWitt H E 2000 Phys. Rev. Lett. 846030

[71] Kalman G J, Hartmann P, Donkó Z and Rosenberg M 2004 Phys. Rev. Lett. 92065001

[72] Rosenberg M and Kalman G J 1997 Phys. Rev. E 567166

[73] Golden K I, Mahassen H and Kalman G J 2004 Phys. Rev. E 70026406

[74] Golden K I, Mahassen H, Kalman G J and Senatore G 2005 Phys. Rev. E 71036401

[75] Golden K I, Mahassen H, Senatore G and Rapisarda F 2006 Phys. Rev. E 74056405

[76] Kalman G J, Golden K I, Donkó Z and Hartmann P 2005 J. Phys.: Conf. Ser. 11254

[77] Maradudin A A 1966 in Solid State Physics; Theoretical and Experimental Aspects of the Effects of Point Defects and Disorder on the Vibrations of Crystals - I vol 18 (New York: Academic Press, eds. Seitz F and Turnbull D) pp 273-420

[78] Maradudin A A 1966 in Solid State Physics; Theoretical and Experimental Aspects of the Effects of Point Defects and Disorder on the Vibrations of Crystals - II vol 19 (New York: Academic Press, eds. Seitz F and Turnbull D) pp 1-134

[79] Sullivan T, Kalman G J, Kyrkos S, Bakshi P, Rosenberg M and Donkó Z 2006 J. Phys. A: Math. Gen. 394607

[80] Peeters F M and W X 1987 Phys. Rev. A 353109

[81] Wang X, Bhattacharjee A and Hu S 2001 Phys. Rev. Lett. 862569

[82] Hartmann P, Donkó Z, Kalman G J, Kyrkos S, Rosenberg M and Bakshi P 2007 IEEE Trans. Plasma Sci. 35337

[83] Sullivan T, Kalman G J and Kyrkos S /unpublished/

[84] Quinn R A and Goree J 2002 Phys. Rev. Lett. 88195001

[85] Nunomura S, Samsonov D and Goree J 2000 Phys. Rev. Lett. 845141

[86] Brout R 1959 Phys. Rev. 11343

[87] Saigo T and Hamaguchi S 2003 Phys. Plasmas 91210

[88] Lampe M, Joyce G, Ganguli G and Gavrishchaka V 2000 Phys. Plasmas 73851

[89] Rabani E, Gezelter J D and Berne B J 1997 J. Chem. Phys. 1076867

[90] Lai Y J and I L 2002 Phys. Rev. Lett. 89155002

[91] Daligault J 2006 Phys. Rev. Lett. 96065003

[92] Hamaguchi S and Ohta H 2000 J. Phys. IV 10 Pr5-19

[93] Ohta H and Hamaguchi S 2000 Phys. Rev. Lett. 846026

[94] Ohta H and Hamaguchi S 2000 Phys. Plasmas 74506

[95] Totsuji H and Kakeya N 1980 Phys. Rev. A 221220

[96] Schmidt P, Zwicknagel G, Reinhard P G and Toepffer C 1997 Phys. Rev. E 567310

[97] Donkó Z, Hartmann P, Kalman G J and Rosenberg M 2003 Contrib. Plasma Phys. 43282

[98] Golden K I, Kalman G J and Wyns P 1990 Phys. Rev. A 416940

[99] Alder B J and Wainwright T E 1970 Phys. Rev. A 118

[100] Liu B and Goree J 2007 Phys. Rev. E 75016405

[101] Liu B and Goree J 2008 Phys. Rev. Lett. 100055003

[102] Ott T, Donkó Z, Hartmann P and Bonitz M to appear in Phys. Rev. E

[103] Donkó Z, Goree J, Hartmann P and Liu B in preparation

[104] Hartmann P, Donkó Z, Bakshi P, Kalman G J and Kyrkos S 2007 IEEE Trans. Plasma Sci. 35 332

[105] Dubin D H E 1993 Phys. Rev. Lett. 712753

[106] Totsuji H, Kishimoto T, Inoue Y, Totsuji C and Nara S 1996 Phys. Lett. A 221215

[107] Bystrenko O 2003 Phys. Rev. E. 67025401

[108] Qiao K and Hyde T W 2005 Phys. Rev. E 71026406

[109] Donkó Z, Kalman G J, Hartmann P, Golden K I and Kutasi K 2003 Phys. Rev. Lett. 90226804

[110] Kalman G J, Donkó Z and Golden K I 2001 Contrib. Plasma. Phys. 41191

[111] Golden K I and Kalman G J 2003 J. Phys. A: Math. Gen. 365865 
[112] Qiao K and Hyde T W 2003 Phys. Rev. E 68046403

[113] Donkó Z, Hartmann P and Kalman G J 2004 Phys. Rev. E 69065401

[114] Vladimirov S V, Shevchenko P V and Cramer N F 1997 Phys. Rev. E 56 R74

[115] Barkan A, Merlino R L and D'Angelo N 1995 Physics of Plasmas 23563

[116] Thompson C, Barkan A, D'Angelo N and Merlino R L 1997 Physics of Plasmas 42331

[117] Pieper J B and Goree J 1996 Phys. Rev. Lett. 773137

[118] Bandyopadhyay P, Prasada G, Sena A and Kaw P K 2007 Phys. Lett. A 386491

[119] Annibaldi S V, Ivlev A V, Konopka U, Ratynskaia S, Thomas H M, Morfill G E, Lipaev A M, Molotkov V I, Petrov O F and Fortov V E 2007 New Journal of Physics 9327

[120] Homann A, Melzer A, Peters S and Piel A 1997 Phys. Rev. E 567138

[121] Peters S, Homann A, Melzer A and Piel A 1996 Physics Letters A 223389

[122] Rao N N, Shukla P K and Yu M Y 1990 Planetary and Space Sci. 38543

[123] Melandso F 1996 Physics of Plasmas 33890

[124] Pramanik J, Prasad G, Sen A and Kaw P K 2002 Phys. Rev. Lett. 88175001

[125] Lemons D S, Murillo M S, Daughton W and Winske D 2000 Physics of Plasmas 72306

[126] Lapenta G 2002 Phys. Rev. E 66026409

[127] Morfill G E, Thomas H M, Konopka U and Zuzic M 1999 Physics of Plasmas 61769

[128] Steinberg V, Sütterlin R, Ivlev A V and Morfill G 2001 Phys. Rev. Lett. 864540

[129] Hebner G A, Riley M E and Marder B M 2003 Phys. Rev. E 68016403

[130] Hebner G A and Riley M E 2004 Phys. Rev. E 69026405

[131] Joyce G, Lampe M and Ganguli G 2002 Phys. Rev. Lett. 88095006

[132] Homann A, Melzer A, Peters S, Madani R and Piel A 1998 Phys. Lett. A 242173

[133] Samsonov D, Goree J, Ma Z W, Bhattacharjee A, Thomas H M and Morfill G E 1999 Phys. Rev. Lett. 833649

[134] Samsonov D, Goree J, Thomas H M and Morfill G E 2000 Phys. Rev. E 615557

[135] Melzer A, Nunomura S, Samsonov D, Ma Z W and Goree J 2000 Phys. Rev. E 624162

[136] Nosenko V, Goree J, Ma Z W and Piel A 2002 Phys. Rev. Lett. 88135001

[137] Nunomura S, Goree J, Hu S, Wang X and Bhattacharjee A 2002 Phys. Rev. E 65066402

[138] Nunomura S, Goree J, Hu S, Wang X, Bhattacharjee A and Avinash K 2002 Phys. Rev. Lett. 89 035001

[139] Schwabe M, Rubin-Zuzic M, Zhdanov S, Thomas H M and Morfill G E 2007 Phys. Rev. Lett. 99 095002

[140] Zhdanov S, Nunomura S, Samsonov D and Morfill G E 2003 Phys. Rev. E 68035401

[141] Melzer A 2003 Phys. Rev. E 67016411

[142] Czahor A 1986 Acta Phys. Pol. A 69281

[143] Misawa T, Ohno N, Asano K, Sawai M, Takamura S and Kaw P K 2001 Phys. Rev. Lett. 861219

[144] Liu B, Avinash K and Goree J 2003 Phys. Rev. Lett 91255003

[145] Liu B and Goree J 2005 Phys. Rev. E 71046410

[146] Nunomura S, Misawa T, Ohno N and Takamura S 1999 Phys. Rev. Lett. 831970

[147] Samsonov D, Zhdanov S and Morfill G 2005 Phys. Rev. E 71026410

[148] Arp O, Block D and Piel A 2004 Phys. Rev. Lett. 93165004

[149] Sheridan T E 2007 Physics of Plasmas 14032108

[150] Messina R and Löwen H 2003 Phys. Rev. Lett. 91146101

[151] Matthews L S, Qiao K and Hyde T W 2006 Adv. Space Research 382564

[152] Smith B, Hyde T, Matthews L, Reay J, Cook M and Schmoke J 2008 Adv. Space Research 41 1509

[153] Goldoni G and Peeters F M 1996 Phys. Rev. B 534591

[154] Assoud L, Messina R and Löwen H ArXiv:0801.1453v1 [cond-mat.soft]

[155] Kalman G J, Valtchinov V and Golden K I 1999 Phys. Rev. Lett. 823124

[156] Golden K I and Kalman G J 1992 Phys. Rev. B 455834

[157] Jiang K, Song Y H and Wang Y N 2007 Physics of Plasmas 14103708 
[158] Feldmann J D, Kalman G J, Hartmann P and Rosenberg M 2008 Phys. Rev. Lett. 100085001

[159] Feldmann J D, Kalman G J and Rosenberg M 2006 J. Phys. A: Math. Gen. 394549

[160] Murillo M S 2000 Phys. Rev. E 624115

[161] Sanbonmatsu K Y and Murillo M S 2001 Phys. Rev. Lett. 861215

[162] Salin G and Caillol J M 2002 Phys. Rev. Lett. 88065002

[163] Salin G and Caillol J M 2003 Phys. Plasmas 101220

[164] Donkó Z and Hartmann P 2004 Phys. Rev. E 69016405

[165] Donkó Z and Hartmann P to appear in Phys. Rev. E

[166] Faussurier G and Murillo M S 2003 Phys. Rev. E 67046404

[167] Faussurier G 2004 Phys. Rev. E 69066402

[168] Ramazanov T S and Dzhumagulova K N 2008 Contrib. Plasma Phys. 48357

[169] Vaulina O S, Petrov O F, Gavrikov A V, Adamowich X G and Fortov V E 2008 Phys. Lett. A $\mathbf{3 7 2} 1096$

[170] Liu B, Goree J and Vaulina O S 2006 Phys. Rev. Lett. 96015005

[171] Ramazanov T S, Dzhumagulova K N, Petrov O F and Gavrikov A V 2006 Europhysics Conference Abstracts Vol. 30I; Proc. of 33rd EPS Conference on Plasma Phys. Rome, 19 - 23 June p P4.031

[172] Donkó Z, Goree J, Hartmann P and Kutasi K 2006 Phys. Rev. Lett. 96145003

[173] Nunomura S, Samsonov D, Zhdanov S and Morfill G 2005 Phys. Rev. Lett. 95025003

[174] Nosenko V, Zhdanov S, Ivlev A V, Morfill G, Goree J and Piel A 2008 Phys. Rev. Lett. 100 025003 